\title{
Near-horizon analysis of $D=5$ BPS black holes and rings
}

\author{
Bernard de Wit and Stefanos Katmadas \\ Institute for Theoretical Physics and Spinoza Institute, \\ Utrecht University, Utrecht, The Netherlands \\ E-mail: B.deWit@uu.nl, S.Katmadas@uu.nl
}

ABSTRACT: A comprehensive analysis is presented based exclusively on near-horizon data to determine the attractor equations and the entropy of BPS black holes and rings in five space-time dimensions, for a Lagrangian invariant under eight supersymmetries with higher-derivative couplings. For spinning black holes the results only partially agree with the results of previous work, where often additional input was used beyond the nearhorizon behaviour. A number of discrepancies remains, for example, pertaining to small black holes and to large spinning black holes, which are related to the presence of the higher-derivative couplings. Arguments are presented to explain some of them. For the black rings, the analysis is intricate due to the presence of Chern-Simons terms and due to the fact that the gauge fields are not globally defined. The contributions from the higherderivative couplings take a systematic form in line with expectations based on a variety of arguments.

KeYwords: Black Holes in String Theory, Black Holes, Supergravity Models

ARXIV EPRINT: 0910.4907 


\section{Contents}

1 Introduction 1

2 Superconformal multiplets $\quad 5$

2.1 The Weyl multiplet $\quad 6$

2.2 The vector supermultiplet 9

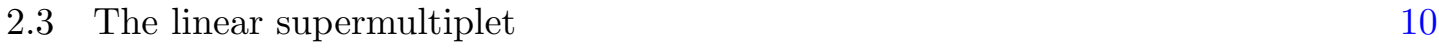

$\begin{array}{lll}2.4 & \text { Hypermultiplets } & 10\end{array}$

3 Tensor calculus $\quad 11$

4 BPS attractor equations 14

$\begin{array}{lll}4.1 & \text { Supersymmetry } & 15\end{array}$

4.2 Space-time geometry 16

4.3 Gauge fields 21

4.4 Linear multiplets 24

5 The Lagrangian and the electric charges 25

6 Entropy and angular momentum for black holes and rings 29

6.1 An alternative form of the mixed Chern-Simons term 31

7 Spinning BPS black holes $\quad 34$

$\begin{array}{lll}8 & \text { BPS black rings } & 39\end{array}$

$\begin{array}{ll}\text { A Conventions } & 46\end{array}$

B Conformal supergravity in 5 space-time dimensions $\quad 47$

\section{Introduction}

The attractor phenomenon for BPS black branes [1-3] is caused by full supersymmetry enhancement at the horizon, which induces stringent restrictions on the values of the fields and the space-time geometry. When supersymmetry is realized off shell, the resulting attractor equations can be analyzed in a way that is independent of the action. In this way universal results can be obtained even when the action contains higher-derivative couplings, as was first demonstrated for $N=2$ supergravity in four dimensions [4].

In five space-time dimensions, supersymmetric attractors come in two varieties, associated with the near-horizon geometry of the rotating black hole $[5,6]$, and of the black 
ring [7]. In the context of the two-derivative effective action, these attractors have been studied in [6, 8-12], using mostly on-shell methods. It is possible to include higherderivative couplings into the conventional two-derivative supergravity action, but those require the use of off-shell methods. Such a (four-derivative) supersymmetric action has been constructed in [13]. Both the two- and the four-derivative couplings involve a ChernSimons term, which is a characteristic feature of five-dimensional supergravity. In the two-derivative case the Chern-Simons term is cubic in the gauge fields, whereas the higherderivative mixed Chern-Simons term involves also the spin connection field. As a result the Lagrangian is only gauge invariant up to a total derivative, a feature that causes certain technical complications.

A study of BPS black holes and black rings that includes these higher-derivative interactions was initiated some time ago in [14-17]. In these works, a number of black hole solutions was constructed, and the corresponding attractors were studied by taking the near-horizon limit. In addition, the entropy function formalism [18] was used to determine the macroscopic entropy of these black holes, after reducing to four dimensions to restore gauge invariance of the action. A corresponding analysis for black rings was hampered by the difficulty in obtaining full asymptotically flat solutions.

In this work, we present a comprehensive treatment of five-dimensional $N=2$ attractors in the presence of the same four-derivative couplings, using the tools provided by the offshell calculus. This analysis of the near-horizon behaviour thus relies only on the full supersymmetry enhancement and does not take into account the more global aspects of possible solutions. In particular, no assumptions are made concerning the existence of interpolating solutions towards asymptotic infinity, and no use is made of any information from outside the near-horizon region. This is in line with the idea that the entropy of black branes should be determined fully by the horizon properties, in the spirit of the BekensteinHawking area law. ${ }^{1}$ As in the four-dimensional analysis, we find that the allowed space-time geometry is the same as for the two-derivative theory, which in the case at hand is described by the $A d S_{2} \times S^{2} \times S^{1}$ geometry of [20]. Because this geometry interpolates between the black hole and the black ring attractors, we can treat both types of five-dimensional attractors in a unified way for a large part of the analysis.

The higher-derivative corrections in the action enter into the expressions for both the entropy and the attractor equations pertaining to electric charges and angular momenta. For the Wald entropy [21-23] we obtain a universal formula expressed in terms of the horizon fields, which applies to both black holes and rings. This is an intriguing result, because the derivation in these two cases proceeds rather differently due to a number of subtleties associated with the mixed gauge-gravitational Chern-Simons term. Our treatment of this mixed Chern-Simons term is inspired by, but not completely identical to, the approach followed in [24]. The existence of a universal entropy formula is in line with previous results based on the entropy function upon reduction to four dimensions, and we confirm this by confronting the results with the four-dimensional near-horizon analysis.

The charges and the angular momenta can also be defined at the horizon. In view of

\footnotetext{
${ }^{1}$ See, however, [19], for a possibly different perspective.
} 
the first law of black hole mechanics, this requires the use of the same Noether potential that enters into the determination of the Wald entropy. The evaluation of the full Noether potential is rather involved, and, as alluded to above, the relevant potentials do not take the same form for black rings and for black holes. The electric charges defined at the horizon are conserved by construction (although they are not invariant under large gauge transformations in the case of black rings). Subtleties arise with the proper definition of the gauge fields in the presence of the Chern-Simons terms, and those have important implications on the attractor equations for black hole and black ring charges.

There exists an extended literature on how to define the electric charges (for a general discussion, see for instance [25]). In the case at hand, it is worth mentioning that the results of this paper differ from those of [15-17]. Many of these differences reside in the definition of the electric charges that was adopted in these references, which was based on the asymptotic fall-off of the electric fields at spatial infinity. As a result the charges for asymptotically Taub-NUT solutions are different from the charges for asymptotically flat solutions, and furthermore these charges depend on the distance from the horizon. In contrast, the charges employed in this paper are insensitive to the asymptotic structure of the space-time and do not depend on the distance from the horizon.

As mentioned above, the BPS near-horizon geometries come in two varieties. In the case of a spacelike horizon cross section with spherical topology, we recover the $A d S_{2} \times S^{3}$ near-horizon geometry of the rotating black hole [5,6]. In the other case we find the $A d S_{3} \times$ $S^{2}$ near-horizon geometry of the supersymmetric black ring [7]. The latter constitutes a special limit of the generic BPS near-horizon geometry for which the spacelike cross section of the horizon has the topology of $S^{2} \times S^{1}$, as is appropriate for a ring. Unlike the black hole, the black ring carries two independent angular momenta associated with rotations in two orthogonal planes. There are some other new features related to the non-contractible $S^{1}$. The first one concerns the fact that this background allows for non-trivial magnetic charges on the circle, because magnetic charges are not pointlike in five dimensions, but are stringlike objects. Hence the ring carries magnetic dipole charges. The second one concerns the non-trivial moduli associated with Wilson lines along the circle. We present a careful treatment of the gauge fields in this topology, which enables us to recover the correct electric charges and their associated attractor equations, following the strategy of [12]. Using this same strategy we also establish the modified electric charges that are additive.

Our results for the entropy agree with the results of microscopic counting for large black holes [5, 26, 27] and for black rings [28, 29]. So far this agreement holds for static black holes, because at present there exists no analytic expression for the microscopic entropy of rotating black holes. ${ }^{2}$ In contrast, we disagree with the microscopic counting [27] for small black holes, whose macrocsopic entropy depends sensitively on the higher-derivative couplings. This puzzle may be characteristic for small black holes; also in four dimensions, the supergravity description of small black holes was often problematic, although there the problems did not pertain to the leading contribution. For black rings the effect of the

\footnotetext{
${ }^{2}$ In theories with 16 supersymmetries explicit expressions are available [30, 31]. It is an interesting question as to whether there exist asymptotic limits thereof which will agree with the results of this paper.
} 
higher-derivative couplings was not included in [28, 29], but we will exactly reproduce the result known from the $(4,0)$ conformal field theory, which leads to an expression proportional to $\sqrt{c_{L} \hat{q}_{0}}$ (c.f. [32]). Here, $c_{L}$ is the central charge, which can be expressed in the dipole charges and which includes the effect of the higher-derivative correction. Its form is in agreement with arguments based on the $A d S_{3}$ near-horizon geometry [14, 33, 34]. Furthermore, $\hat{q}_{0}$ is an appropriately defined quantity expressed in the angular momenta and the charges. As noted in [35], this quantity is naturally written in terms of the aforementioned modified electric charges that are additive.

On the other hand, our results for rotating black holes only partially agree with the macroscopic results of [15-17], as was already alluded to above in connection with the definition used for the electric charges. Our results also disagree with the prediction of [36] for the first-order contribution from the angular momentum to the black hole entropy, based on the addition of the Euler density to the supergravity action. In this work, also a correction to the black ring entropy was determined based on the Euler density. In hindsight, it is difficult to see how the Euler density could possibly capture, at the same time, all contributions to the entropy, the angular momenta and the electric charges for both black holes and black rings, as it does not include the contributions from the mixed Chern-Simons term, which, especially for black rings, is responsible for subtle effects.

Another issue concerns the connection between corresponding black hole solutions and their associated entropy in four and in five dimensions. This connection is motivated by the fact that the four-dimensional theory can be obtained by dimensional reduction on a circle from the five-dimensional one $[12,37,38]$, although there may be subtleties. One such subtlety, related to the supersymmetry preserved by five-dimensional attractors upon reducing to four dimensions, was already discussed in [39]. Following this reasoning, our five-dimensional attractor equations should be related to the four-dimensional attractors with a specific $R^{2}$-coupling. Indeed, we find agreement with four dimensions in the case of the black ring, except that the quantity $\hat{q}_{0}$ in four dimensions will only depend on the unmodified electric charges. We explain the reason for this fact, which is of topological origin and not related to the presence of higher-derivative couplings. For the case of the rotating black hole, we find a clear discrepancy in the contributions from the higherderivative couplings to the electric charges. A similar, though somewhat different, deviation from the four-dimensional situation was observed in [15-17]. One way to understand the discrepancy in the electric charges follows from the observation that the reduction to four dimensions of the higher-derivative term will involve an extra vector multiplet associated with the Kaluza-Klein photon, which will also be subject to higher-derivative interactions. So far, such interactions have never been considered directly in four dimensions, and in fact the precise form of these four-dimensional couplings is not fully known although there are indications that they should not affect the formula for the Wald entropy for BPS black holes. But most likely they will have an effect on the electric charges, and this may resolve the present discrepancy between the electric charges in four and five dimensions.

This paper is organized as follows. Section 2 contains a concise but comprehensive review of the superconformal transformation rules for the supermultiplets of interest. In section 3 the product rules and supersymmetric density formulae are presented, using the 
notation of this paper. Section 4 is devoted to the derivation of the attractor equations and their consequences. The attractor equations are derived in subsection 4.1, and the resulting geometry is discussed in subsection 4.2. Subsequently the horizon values of the gauge fields and the linear supermultiplets are discussed in subsections 4.3 and 4.4, respectively. The invariant action is given in section 5 from which the attractor equations for the electric charges can be determined. In section 6 we discuss the entropy and angular momentum for black holes and rings. As it turns out the mixed Chern-Simons term requires a different approach for black holes and black rings. Therefore, after a review of the more generic situation for black holes, a subsection 6.1 is devoted to the alternative treatment of the mixed Chern-Simons term that is required for the black rings. The final results of this paper for spinning black holes and black rings, together with a discussion and a comparison to results in the literature, are presented in section 7 and section 8 , respectively. Readers who are not primarily interested in the technical details, may proceed directly to these two sections. There are two appendices: appendix A introduces the spinor and space-time notation used in the first part of this paper, and appendix B contains a brief review of extended conformal supergravities in five space-time dimensions.

\section{Superconformal multiplets}

A convenient method for dealing with off-shell formulations of supergravity theories is provided by the superconformal multiplet calculus. This calculus was originally set up for $N=2$ supergravity in $d=4$ dimensions [40-43], following early work for $N=1, d=4$ supergravity [44,45]. The $N=1$ case was worked out more fully in [46], and shortly thereafter the formalism was also applied to $N=1, d=6$ supergravity in [47]. For $d=5$ dimensions superconformal methods were developed relatively recently by several groups [13, 48-50], and these results were exploited in the work of $[14,15,17]$. However, these groups use different field and symmetry definitions, which have features that are qualitatively different from the conventions used in $d=4$ dimensions. Obviously this poses no problem of principle, but in order to make the connection with the four-dimensional theory as direct as possible, we have chosen to adopt slightly different conventions.

In this section we give a self-contained summary of the transformation rules of superconformal multiplets in five space-time dimensions, namely the Weyl multiplet, the vector multiplet, the linear multiplet and the hypermultiplet for supergravity in five space-time dimensions with eight supercharges. With the exception of the hypermultiplet, these multiplets define off-shell representations of the algebra of superconformal transformations. We refer to appendix A for spinor and space-time conventions. Some additional material about the Weyl multiplets in four and five dimensions with eight and sixteen supercharges is presented in appendix B. The fields of conformal supergravity are dual to the components of the supermultiplet of currents, and are subject to a number of gauge transformations directly related to the conservation laws of these currents. The bosonic gauge transformations are those of the conformal group, diffeomorphisms, local Lorentz transformations with generators $M_{a b}$, scale transformations with generator $D$ and special conformal transformations (also called conformal boosts) with generators $K_{a}$. Furthermore there are local 
R-symmetry transformations. In five space-time dimensions, the R-symmetry group equals $\mathrm{USp}(2 N)$ so that for simple supergravity we have $\mathrm{USp}(2) \cong \mathrm{SU}(2)$. The fermionic gauge transformations are the conventional Q- and the special conformal S-supersymmetry transformations.

\subsection{The Weyl multiplet}

The Weyl multiplet of five-dimensional simple conformal supergravity is shown in table 3 . The independent fields consist of the fünfbein $e_{\mu}{ }^{a}$, the gravitino field $\psi_{\mu}{ }^{i}$, the dilatational gauge field $b_{\mu}$, the R-symmetry gauge fields $V_{\mu i}{ }^{j}$ (which is an anti-hermitean, traceless matrix in the $\mathrm{SU}(2)$ indices $i, j$ ) and a tensor field $T_{a b}$, a scalar field $D$ and a spinor field $\chi^{i}$. The three gauge fields $\omega_{\mu}^{a b}, f_{\mu}^{a}$ and $\phi_{\mu}$, associated with local Lorentz transformations, conformal boosts and S-supersymmetry, respectively, are not independent and will be discussed later. The infinitesimal Q, S and K transformations of the independent fields, parametrized by spinors $\epsilon^{i}$ and $\eta^{i}$ and a vector $\Lambda_{\mathrm{K}}{ }^{a}$, respectively, are as follows,

$$
\begin{aligned}
\delta e_{\mu}{ }^{a}= & \frac{1}{2} \bar{\epsilon}_{i} \gamma^{a} \psi_{\mu}{ }^{i} \\
\delta \psi_{\mu}^{i}= & \mathcal{D}_{\mu} \epsilon^{i}+\frac{1}{4} \mathrm{i} T_{a b}\left(3 \gamma^{a b} \gamma_{\mu}-\gamma_{\mu} \gamma^{a b}\right) \epsilon^{i}-\mathrm{i} \gamma_{\mu} \eta^{i} \\
\delta V_{\mu i}{ }^{j}= & 3 \mathrm{i} \bar{\epsilon}_{i} \phi_{\mu}{ }^{j}-8 \bar{\epsilon}_{i} \gamma_{\mu} \chi^{j}-3 \mathrm{i} \bar{\eta}_{i} \psi_{\mu}{ }^{j}+\delta^{i}{ }_{j}\left[-\frac{3}{2} \mathrm{i} \bar{\epsilon}_{k} \phi_{\mu}{ }^{k}+4 \bar{\epsilon}_{k} \gamma_{\mu} \chi^{k}+\frac{3}{2} \mathrm{i} \bar{\eta}_{k} \psi_{\mu}^{k}\right], \\
\delta b_{\mu}= & \frac{1}{2} \mathrm{i} \bar{\epsilon}_{i} \phi_{\mu}^{i}-2 \bar{\epsilon}_{i} \gamma_{\mu} \chi^{i}+\frac{1}{2} \mathrm{i} \bar{\eta}_{i} \psi_{\mu}^{i}+2 \Lambda_{K}{ }^{a} e_{\mu a}, \\
\delta T_{a b}= & \frac{2}{3} \mathrm{i} \bar{\epsilon}_{i} \gamma_{a b} \chi^{i}-\frac{1}{8} \mathrm{i} \bar{\epsilon}_{i} R_{a b}{ }^{i}(Q) \\
\delta \chi^{i}= & \frac{1}{4} \epsilon^{i} D+\frac{1}{128} R_{\mu \nu j}{ }^{i}(V) \gamma^{\mu \nu} \epsilon^{j}+\frac{3}{128} \mathrm{i}\left(3 \gamma^{a b} \not D+\not D \gamma^{a b}\right) T_{a b} \epsilon^{i} \\
& -\frac{3}{32} T_{a b} T_{c d} \gamma^{a b c d} \epsilon^{i}+\frac{3}{16} T_{a b} \gamma^{a b} \eta^{i} \\
\delta D= & \bar{\epsilon}_{i} \not D \chi^{i}-\mathrm{i} \bar{\epsilon}_{i} T_{a b} \gamma^{a b} \chi^{i}-\mathrm{i} \bar{\eta}_{i} \chi^{i} .
\end{aligned}
$$

Under local scale transformations the various fields and transformation parameters transform as indicated in table 1 . The derivatives $\mathcal{D}_{\mu}$ are covariant with respect to all the bosonic gauge symmetries with the exception of the conformal boosts. In particular we note

$$
\mathcal{D}_{\mu} \epsilon^{i}=\left(\partial_{\mu}-\frac{1}{4} \omega_{\mu}{ }^{c d} \gamma_{c d}+\frac{1}{2} b_{\mu}\right) \epsilon^{i}+\frac{1}{2} V_{\mu j}{ }^{i} \epsilon^{j}
$$

where the gauge fields transform under their respective gauge transformations according to $\delta \omega_{\mu}{ }^{a b}=\mathcal{D}_{\mu} \lambda^{a b}, \delta b_{\mu}=\mathcal{D}_{\mu} \Lambda_{D}$ and $\delta V_{\mu i}{ }^{j}=\mathcal{D}_{\mu} \Lambda_{i}{ }^{j}$, with $\left(\Lambda_{i}{ }^{j}\right)^{*} \equiv \Lambda^{i}{ }_{j}=-\Lambda_{j}{ }^{i}$. The derivatives $D_{\mu}$ are covariant with respect to all the superconformal symmetries.

In order to discuss the dependent gauge fields, we first introduce the following curvature 


\begin{tabular}{|c|ccccccc|ccc|cc|}
\hline & \multicolumn{7}{|c|}{ Weyl multiplet } & \multicolumn{2}{|c|}{ parameters } \\
\hline field & $e_{\mu}{ }^{a}$ & $\psi_{\mu}{ }^{i}$ & $b_{\mu}$ & $\mathcal{V}_{\mu i}{ }^{j}$ & $T_{a b}$ & $\chi^{i}$ & $D$ & $\omega_{\mu}{ }^{a b}$ & $f_{\mu}{ }^{a}$ & $\phi_{\mu}{ }^{i}$ & $\epsilon^{i}$ & $\eta^{i}$ \\
\hline$w$ & -1 & $-\frac{1}{2}$ & 0 & 0 & 1 & $\frac{3}{2}$ & 2 & 0 & 1 & $\frac{1}{2}$ & $-\frac{1}{2}$ & $\frac{1}{2}$ \\
\hline
\end{tabular}

Table 1. Weyl weights $w$ of the Weyl multiplet component fields and the supersymmetry transformation parameters.

tensors,

$$
\begin{aligned}
& R_{\mu \nu}{ }^{a}(P)=2 \mathcal{D}_{[\mu} e_{\nu]}^{a}-\frac{1}{2} \bar{\psi}_{[\mu i} \gamma^{a} \psi_{\nu]}{ }^{i}, \\
& R_{\mu \nu}^{a b}(M)=2 \partial_{[\mu} \omega_{\nu]}^{a b}-2 \omega_{[\mu}^{a c} \omega_{\nu] c}^{b}-8 e_{[\mu}^{[a} f_{\nu]}^{b]}+\mathrm{i} \bar{\psi}_{[\mu i} \gamma^{a b} \phi_{\nu]}^{i} \\
& -\frac{1}{4} \mathrm{i} T^{c d} \bar{\psi}_{[\mu i}\left(6 \gamma^{[a} \gamma_{c d} \gamma^{b]}-\gamma^{a b} \gamma_{c d}-\gamma_{c d} \gamma^{a b}\right) \psi_{\nu]}^{i} \\
& -\frac{1}{2} \bar{\psi}_{[\mu i}\left(\gamma_{\nu]} R^{a b i}(Q)+2 \gamma^{[a} R_{\nu]}^{b] i}(Q)\right)+8 e_{[\mu}^{[a} \bar{\psi}_{\nu] i} \gamma^{b]} \chi^{i}, \\
& R_{\mu \nu}(D)=2 \partial_{[\mu} b_{\nu]}-4 f_{[\mu}{ }^{a} e_{\nu] a}-\mathrm{i} \bar{\psi}_{[\mu i} \phi_{\nu]}^{i}+4 \bar{\psi}_{[\mu i} \gamma_{\nu]} \chi^{i} \text {. } \\
& R_{\mu \nu i}{ }^{j}(V)=2 \partial_{[\mu} V_{\nu] i}{ }^{j}-V_{[\mu i}{ }^{k} V_{\nu] k}{ }^{j} \\
& -6 \mathrm{i} \bar{\psi}_{[\mu i} \phi_{\nu]}{ }^{j}+16 \bar{\psi}_{[\mu i} \gamma_{\nu]} \chi^{j}+\delta_{i}^{j}\left[3 \mathrm{i} \bar{\psi}_{[\mu k} \phi_{\nu]}^{k}-8 \bar{\psi}_{[\mu k} \gamma_{\nu]} \chi^{k}\right], \\
& R_{\mu \nu}{ }^{i}(Q)=2 \mathcal{D}_{[\mu} \psi_{\nu]}{ }^{i}-2 \mathrm{i} \gamma_{[\mu} \phi_{\nu]}{ }^{i}+\frac{1}{2} \mathrm{i} T_{a b}\left(3 \gamma^{a b} \gamma_{[\mu}-\gamma_{[\mu} \gamma^{a b}\right) \psi_{\nu]}{ }^{i}
\end{aligned}
$$

The conventional constraints (which are not invariant under Q- and S-supersymmetry) are as follows,

$$
\begin{aligned}
R_{\mu \nu}{ }^{a}(P) & =0, \\
\gamma^{\mu} R_{\mu \nu}{ }^{i}(Q) & =0, \\
e_{a}{ }^{\mu} R_{\mu \nu}^{a b}(M) & =0 .
\end{aligned}
$$

These conditions determine the gauge fields $\omega_{\mu}{ }^{a b}, f_{\mu}{ }^{a}$ and $\phi_{\mu}{ }^{i}$. The conventional constraints lead to additional constraints on the curvatures when combined with the Bianchi identities. In this way one derives $R_{[a b c] d}(M)=0=R_{a b}(D)$ and the pair-exchange property $R_{a b c d}=$ $R_{c d a b}$ from the first and the third constraint. The second constraint, which implies also that $\gamma_{[\mu \nu} R_{\rho \sigma]}{ }^{i}(Q)=0$, determines the curvature $R_{\mu \nu}{ }^{i}(S)$, which we refrained from defining previously. It turns out to be proportional to $R_{\mu \nu}{ }^{i}(Q)$ and derivatives thereof,

$$
\begin{aligned}
R_{\mu \nu}{ }^{i}(S)= & -\mathrm{i} \not D R_{\mu \nu}{ }^{i}(Q)-\mathrm{i} \gamma_{[\mu} D^{\rho} R_{\nu] \rho}^{i}(Q)-4 \gamma_{\mu \nu} T^{\rho \sigma} R_{\rho \sigma}{ }^{i}(Q) \\
& +18 \gamma_{\sigma} T^{\rho \sigma} \gamma_{[\mu} R_{\nu] \rho}^{i}(Q)-5 T^{\rho \sigma} \gamma_{\rho \sigma} R_{\mu \nu}{ }^{i}(Q)-12 T_{[\mu}^{\rho} R_{\nu] \rho}^{i}(Q) .
\end{aligned}
$$

The remaining curvature $R_{\mu \nu}{ }^{a}(K)$ does not play a role in what follows.

Whereas the first constraint is invariant under S- but not under Q-supersymmetry, the other two constraints are invariant under neither supersymmetry. This implies that the dependent gauge fields will acquire terms in their transformation rules proportional to the 
constrained curvature tensors,

$$
\begin{aligned}
\delta \omega_{\mu}{ }^{a b}= & \mathcal{D}_{\mu} \lambda^{a b}+4 \Lambda_{K}{ }^{[a} e_{\mu}{ }^{b]}-\frac{1}{2} \mathrm{i} \epsilon_{i} \gamma^{a b} \phi_{\mu}{ }^{i}+\frac{1}{2} \mathrm{i} \eta_{i} \gamma^{a b} \psi_{\mu}{ }^{i} \\
& +\frac{1}{8} \mathrm{i} T^{c d} \bar{\epsilon}_{i}\left(6 \gamma^{[a} \gamma_{c d} \gamma^{b]}-\gamma^{a b} \gamma_{c d}-\gamma_{c d} \gamma^{a b}\right) \psi_{\mu}{ }^{i} \\
& +\frac{1}{4} \bar{\epsilon}_{i}\left(\gamma_{\mu} R^{a b i}(Q)+2 \gamma^{[a} R_{\mu}{ }^{b] i}(Q)\right)+4 e_{\mu}{ }^{[a} \bar{\epsilon}_{i} \gamma^{b]} \chi^{i}, \\
\delta \phi_{\mu}{ }^{i}= & \mathcal{D}_{\mu} \eta^{i}+\frac{1}{4} \mathrm{i} T_{a b}\left(\gamma_{\mu} \gamma^{a b}-\gamma^{a b} \gamma_{\mu}\right) \eta^{i}+\mathrm{i} f_{\mu}{ }^{a} \gamma_{a} \epsilon^{i}-\mathrm{i} \Lambda_{K}{ }^{a} \gamma_{a} \psi_{\mu}{ }^{i} \\
& -\frac{1}{48} \mathrm{i}\left(2 \gamma^{a b} \gamma_{\mu}-\gamma_{\mu} \gamma^{a b}\right) R_{a b j}{ }^{i}(V) \epsilon^{j}+\frac{1}{4}\left(\not D T^{a b} \gamma_{a b} \gamma_{\mu}+D_{a} T^{a b} \gamma_{\mu} \gamma_{b}\right) \epsilon^{i} \\
& +\mathrm{i}\left(-\frac{3}{4} T^{a b} T^{c d} \gamma_{\mu a b c d}+T_{\mu a} T_{b c} \gamma^{a b c}-4 T_{\mu a} T^{a b} \gamma_{b}-\frac{3}{4} \gamma_{\mu} T^{2}\right) \epsilon^{i} \\
& -\frac{9}{4} \mathrm{i} \bar{\epsilon}_{j} \psi_{\mu}{ }^{j} \chi^{i}+\frac{7}{4} \mathrm{i} \bar{\epsilon}_{j} \gamma_{a} \psi_{\mu}{ }^{j} \gamma^{a} \chi^{i}-\frac{1}{8} \mathrm{i} \bar{\epsilon}_{k} \gamma^{a b} \psi_{\mu}{ }^{k}\left(\gamma_{a b} \chi^{i}+\frac{1}{4} R_{a b}{ }^{i}(Q)\right) \\
& +\frac{1}{4} \mathrm{i} \bar{\epsilon}_{k} \gamma^{a b} \psi_{\mu}{ }^{i}\left(\gamma_{a b} \chi^{k}+\frac{1}{4} R_{a b}{ }^{k}(Q)\right), \\
\delta f_{\mu}{ }^{a}= & \mathcal{D}_{\mu} \Lambda_{K}{ }^{a}+\frac{1}{2} \eta_{i} \gamma^{a} \phi_{\mu}{ }^{i}+\cdots,
\end{aligned}
$$

where here and henceforth $T^{2} \equiv\left(T_{a b}\right)^{2}$. With these results we obtain the following Q- and $\mathrm{S}$-variations that will be needed in due course,

$$
\begin{aligned}
\delta R_{a b}{ }^{i}(Q)= & -\frac{1}{24}\left(\gamma^{c d} \gamma_{a b}-4 \delta_{a}{ }^{[c} \delta_{b}{ }^{d]}\right) R_{c d i}{ }^{i}(V) \epsilon^{j}-\frac{1}{4} R_{a b}{ }^{c d}(M) \gamma_{c d} \epsilon^{i} \\
& +\frac{1}{2} \mathrm{i}\left(3 D_{[a} T^{c d} \gamma_{c d} \gamma_{b]}-D_{[a} T^{c d} \gamma_{b]} \gamma_{c d}-\gamma_{[a} \not D T^{c d} \gamma_{c d} \gamma_{b]}-D_{c} T^{c d} \gamma_{a b} \gamma_{d}\right) \epsilon^{i} \\
& -2\left(T_{a b} T_{c d} \gamma^{c d}+T_{a c} T_{b d} \gamma^{c d}+2 T_{c[a} T^{c d} \gamma_{b] d}+\frac{1}{4} T^{2} \gamma_{a b}\right) \epsilon^{i} \\
& \left.+\left(\gamma^{c d} \gamma_{a b}-4 \delta_{a}{ }^{[c} \delta_{b} d\right]\right) \eta^{i} T_{c d}, \\
\delta R_{a b i}{ }^{j}(V)= & 3 \mathrm{i} \bar{\epsilon}_{i} R_{a b}{ }^{j}(S)+16 \bar{\epsilon}_{i} \gamma_{[a} D_{b]} \chi^{j}-4 \mathrm{i} \bar{\epsilon}_{i}\left(3 \gamma_{[a} \gamma^{c d} \gamma_{b]}-\gamma^{c d} \gamma_{a b}\right) \chi^{j} T_{c d} \\
& -3 \mathrm{i} \bar{\eta}_{i} R_{a b}{ }^{j}(Q)-16 \mathrm{i} \bar{\eta}_{i} \gamma_{a b} \chi^{j}-\text { trace } .
\end{aligned}
$$

The above transformations coincide with those of $[48,50]$, upon including a $T$ dependent S-supersymmetry transformation into the Q-supersymmetry variations and rescaling the tensor field by a factor $4 / 3$. The difference with the conventions of $[13,49]$ are a bit more involved. The commutator of two Q-supersymmetry transformations closes into the superconformal transformations as follows,

$$
\left[\delta_{Q}\left(\epsilon_{1}\right), \delta_{Q}\left(\epsilon_{2}\right)\right]=\xi^{\mu} \hat{D}_{\mu}+\delta_{M}(\lambda)+\delta_{S}(\eta)+\delta_{K}\left(\Lambda_{K}\right),
$$

where $\xi^{\mu} \hat{D}_{\mu}$ denotes the effect of a supercovariant general coordinate transformation. The parameters appearing on the right-hand side associated with the general coordinate transformation and the Lorentz transformation are given by

$$
\begin{aligned}
\xi^{\mu} & =\frac{1}{2} \bar{\epsilon}_{2 i} \gamma^{\mu} \epsilon_{1}{ }^{i}, \\
\lambda^{a b} & =\frac{1}{8} \mathrm{i} T^{c d} \bar{\epsilon}_{2 i}\left(6 \gamma^{[a} \gamma_{c d} \gamma^{b]}-\gamma^{a b} \gamma_{c d}-\gamma_{c d} \gamma^{a b}\right) \epsilon_{1}{ }^{i},
\end{aligned}
$$


whereas the parameters associated with S-supersymmetry and conformal boosts are not given as they are not needed below. The commutator of two S-supersymmetry transformations and the commutator of a Q- and an S-supersymmetry transformation close as follows,

$$
\begin{aligned}
{\left[\delta_{S}\left(\eta_{1}\right), \delta_{S}\left(\eta_{2}\right)\right] } & =\delta_{K}\left(\Lambda_{K}\right) \\
{\left[\delta_{S}(\eta), \delta_{Q}(\epsilon)\right] } & =\delta_{D}\left(\Lambda_{D}\right)+\delta_{M}(\lambda)+\delta_{R}(\Lambda)+\delta_{K}\left(\tilde{\Lambda}_{K}\right),
\end{aligned}
$$

where

$$
\begin{aligned}
\Lambda_{K}{ }^{a} & =\frac{1}{2} \bar{\eta}_{2 i} \gamma^{a} \eta_{1}{ }^{i}, \\
\Lambda_{D} & =\frac{1}{2} \mathrm{i} \bar{\epsilon}_{i} \eta^{i}, \\
\lambda^{a b} & =-\frac{1}{2} \mathrm{i} \bar{\epsilon}_{i} \gamma^{a b} \eta^{i}, \\
\Lambda_{i}^{j} & =3 \mathrm{i}\left(\bar{\epsilon}_{i} \eta^{j}-\frac{1}{2} \delta_{i}^{j} \bar{\epsilon}_{k} \eta^{k}\right), \\
\tilde{\Lambda}_{K}{ }^{a} & =-\frac{1}{16} \bar{\epsilon}_{i}\left[5 \gamma^{a} \gamma^{b c}-4 \gamma^{b c} \gamma^{a}\right] \eta^{i} T_{b c} .
\end{aligned}
$$

Furthermore, we note the following commutation relation,

$$
\left[\delta_{K}\left(\Lambda_{K}\right), \delta_{Q}(\epsilon)\right]=\delta_{S}\left(\mathrm{i} \mathcal{X}_{K} \epsilon^{i}\right) .
$$

\subsection{The vector supermultiplet}

The vector supermultiplet consists of a real scalar $\sigma$, a gauge field $W_{\mu}$, a triplet of (auxiliary) fields $Y^{i j}$, and a fermion field $\Omega^{i}$. Under superconformal transformations these fields transform as follows,

$$
\begin{aligned}
\delta \sigma & =\frac{1}{2} \mathrm{i} \bar{\epsilon}_{i} \Omega^{i} \\
\delta \Omega^{i} & =-\frac{1}{4}\left(\hat{F}_{a b}-4 \sigma T_{a b}\right) \gamma^{a b} \epsilon^{i}-\frac{1}{2} \mathrm{i} \not D \sigma \epsilon^{i}-\varepsilon_{j k} Y^{i j} \epsilon^{k}+\sigma \eta^{i}, \\
\delta W_{\mu} & =\frac{1}{2} \bar{\epsilon}_{i} \gamma_{\mu} \Omega^{i}-\frac{1}{2} \mathrm{i} \sigma \bar{\epsilon}_{i} \psi_{\mu}^{i}, \\
\delta Y^{i j} & =\frac{1}{2} \varepsilon^{k(i} \bar{\epsilon}_{k} \not D \Omega^{j)}+\mathrm{i} \varepsilon^{k(i} \bar{\epsilon}_{k}\left(-\frac{1}{4} T_{a b} \gamma^{a b} \Omega^{j)}+4 \sigma \chi^{j)}\right)-\frac{1}{2} \mathrm{i} \varepsilon^{k(i} \bar{\eta}_{k} \Omega^{j)} .
\end{aligned}
$$

where $\left(Y^{i j}\right)^{*} \equiv Y_{i j}=\varepsilon_{i k} \varepsilon_{j l} Y^{k l}$, and the supercovariant field strength is defined as,

$$
\hat{F}_{\mu \nu}=\partial_{\mu} W_{\nu}-\partial_{\nu} W_{\mu}-\bar{\Omega}_{i} \gamma_{[\mu} \psi_{\nu]}^{i}+\frac{1}{2} \mathrm{i} \sigma \bar{\psi}_{[\mu i} \psi_{\nu]}{ }^{i} .
$$

The commutator of two Q-supersymmetry transformations closes as in (2.8) modulo an extra gauge transformation, $\delta W_{\mu}=\partial_{\mu}\left(\frac{1}{2} \mathrm{i} \sigma \bar{\epsilon}_{2 i} \epsilon_{1}{ }^{i}\right)$. We also note the transformation rule,

$$
\begin{aligned}
\delta\left(\hat{F}_{a b}-4 \sigma T_{a b}\right)= & -\bar{\epsilon}_{i} \gamma_{[a} D_{b]} \Omega^{i}-\frac{8}{3} \sigma \bar{\epsilon}_{i} \gamma_{a b} \chi^{i} \\
& +\frac{1}{4} \mathrm{i} \bar{\epsilon}_{i}\left(3 \gamma_{[a} \gamma^{c d} \gamma_{b]}-\gamma^{c d} \gamma_{a b}-8 \delta_{a}^{c} \delta_{b}^{d}\right) \Omega^{i} T_{c d}+\mathrm{i} \bar{\eta}_{i} \gamma_{a b} \Omega^{i} .
\end{aligned}
$$

The fields behave under local scale transformations according to the weights shown in table 2 . 


\begin{tabular}{|c|cccc|}
\hline & \multicolumn{4}{|c|}{ vector multiplet } \\
\hline field & $\sigma$ & $W_{\mu}$ & $\Omega_{i}$ & $Y_{i j}$ \\
\hline$w$ & 1 & 0 & $\frac{3}{2}$ & 2 \\
\hline & \multicolumn{4}{|c|}{ linear multiplet } \\
\hline field & $L^{i j}$ & $E_{a}$ & $\varphi_{i}$ & $N$ \\
\hline$w$ & 3 & 4 & $\frac{7}{2}$ & 4 \\
\hline & \multicolumn{4}{|c|}{ hypermultiplet } \\
\hline field & $A_{i}{ }^{\alpha}$ & \multicolumn{3}{c|}{$\zeta^{\alpha}$} \\
\hline$w$ & $\frac{3}{2}$ & & \\
\hline
\end{tabular}

Table 2. Weyl weights $w$ of the vector multiplet, the tensor (linear) multiplet, and the hypermultiplet component fields.

\subsection{The linear supermultiplet}

The linear multiplet consists of a triplet of scalars $L^{i j}$, a divergence-free vector $\hat{E}_{a}$, an (auxiliary) scalar $N$, and a fermion field $\varphi^{i}$. The superconformal transformation rules for these fields are as follows,

$$
\begin{aligned}
\delta L^{i j} & =-\mathrm{i} \varepsilon^{k(i} \bar{\epsilon}_{k} \varphi^{j)}, \\
\delta \varphi^{i} & =-\frac{1}{2} \mathrm{i} \varepsilon_{j k} \not D L^{i j} \epsilon^{k}+\frac{1}{2}(N-\mathrm{i} \hat{\phi}) \epsilon^{i}+3 \varepsilon_{j k} L^{i j} \eta^{k}, \\
\delta \hat{E}_{a} & =-\frac{1}{2} \mathrm{i} \bar{\epsilon}_{i} \gamma_{a b} D^{b} \varphi^{i}+\frac{1}{8} \bar{\epsilon}_{i}\left(3 \gamma_{a} \gamma^{b c}+\gamma^{b c} \gamma_{a}\right) \varphi^{i} T_{b c}-2 \bar{\eta}_{i} \gamma_{a} \varphi^{i}, \\
\delta N & =\frac{1}{2} \bar{\epsilon}_{i} \not D \varphi^{i}+\frac{3}{4} \mathrm{i} \bar{\epsilon}_{i} \gamma^{a b} \varphi^{i} T_{a b}-4 \mathrm{i} \varepsilon_{j k} \bar{\epsilon}_{i} \chi^{k} L^{i j}+\frac{3}{2} \mathrm{i} \bar{\eta}_{i} \varphi^{i} .
\end{aligned}
$$

The constraint on $\hat{E}^{a}$,

$$
D_{a} \hat{E}^{a}=0,
$$

can be solved in terms of a three-rank anti-symmetric tensor gauge field $E_{\mu \nu \rho}$, which transforms as follows under the superconformal transformations,

$$
\delta E_{\mu \nu \rho}=\frac{1}{2} \bar{\epsilon}_{i} \gamma_{\mu \nu \rho} \varphi^{i}-\frac{3}{2} \mathrm{i} \bar{\epsilon}_{i} \gamma_{[\mu \nu} \psi_{\rho]}^{k} \varepsilon_{j k} L^{i j} .
$$

These transformations close according to the commutation relations (2.8), (2.10) and (2.12), up to a tensor gauge transformation, $\delta E_{\mu \nu \rho}=\partial_{[\mu}\left(-\frac{2}{3} \mathrm{i} \bar{\epsilon}_{2 i} \gamma_{\nu \rho]} \epsilon_{1}{ }^{k} \varepsilon_{j k} L^{i j}\right)$. The supercovariant field strength associated with $E_{\mu \nu \rho}$ equals

$$
\hat{E}^{\mu}=\frac{1}{6} \mathrm{i} e^{-1} \varepsilon^{\mu \nu \rho \sigma \lambda}\left[\partial_{\nu} E_{\rho \sigma \lambda}-\frac{1}{2} \bar{\psi}_{\nu i} \gamma_{\rho \sigma \lambda} \varphi^{i}+\frac{3}{4} \mathrm{i} \bar{\psi}_{\nu i} \gamma_{\rho \sigma} \psi_{\lambda}{ }^{k} \varepsilon_{j k} L^{i j}\right] .
$$

The behaviour under local scale transformations follow from the weights shown in table 2 . The tensor field $E_{\mu \nu \rho}$ is inert under scale transformations and thus carries zero weight.

\subsection{Hypermultiplets}

Hypermultiplets are necessarily associated with target spaces of dimension $4 r$ that are hyperkähler cones $[51,52]$. The supersymmetry transformations are most conveniently 
written in terms of the sections $A_{i}{ }^{\alpha}(\phi)$, where $\alpha=1,2, \ldots, 2 r$,

$$
\begin{aligned}
\delta A_{i}{ }^{\alpha} & =\mathrm{i} \bar{\epsilon}_{i} \zeta^{\alpha}, \\
\delta \zeta^{\alpha} & =-\frac{1}{2} \mathrm{i} \not D A_{i}{ }^{\alpha} \epsilon^{i}+\frac{3}{2} A_{i}{ }^{\alpha} \eta^{i} .
\end{aligned}
$$

The $A_{i}{ }^{\alpha}$ are the local sections of an $\operatorname{Sp}(r) \times \mathrm{Sp}(1)$ bundle. The existence of such an associated bundle is known from general arguments [53]. We also note the existence of a covariantly constant skew-symmetric tensor $\Omega_{\alpha \beta}$ (and its complex conjugate $\Omega^{\alpha \beta}$ satisfying $\Omega_{\alpha \gamma} \Omega^{\beta \gamma}=-\delta_{\alpha}{ }^{\beta}$ ), and the symplectic Majorana condition for the spinors reads as $C^{-1} \bar{\zeta}_{\alpha}{ }^{\mathrm{T}}=\Omega_{\alpha \beta} \zeta^{\beta}$. Covariant derivatives contain the $\operatorname{Sp}(r)$ connection $\Gamma_{A}{ }_{\beta}{ }_{\beta}$, associated with rotations of the fermions. The sections $A_{i}{ }^{\alpha}$ are pseudo-real, i.e. they are subject to the constraint, $A_{i}{ }^{\alpha} \varepsilon^{i j} \Omega_{\alpha \beta}=A^{j}{ }_{\beta} \equiv\left(A_{j}{ }^{\beta}\right)^{*}$. The information on the target-space metric is contained in the so-called hyperkähler potential,

$$
\varepsilon_{i j} \chi=\Omega_{\alpha \beta} A_{i}^{\alpha} A_{j}{ }^{\beta} .
$$

For the local scale transformations we refer again to the weights shown in table 2 . The hypermultiplet does not exist as an off-shell supermultiplet. Closure of the superconformal transformations is only realized upon using fermionic field equations, but this fact does not represent a serious problem in what follows.

\section{Tensor calculus}

In the previous section we introduced various superconformal multiplets. With the exception of the hypermultiplets, these multiplets are truly off-shell, so that the superconformal symmetries close without the need for imposing field equations. The tensor calculus for these multiplets consists of various multiplication rules and decompositions, as well as invariant density formulas. With these results one can construct a rather general class of invariant actions.

In five space-time dimensions the linear multiplets play an important role. At the linearized level in flat space, linear and vector supermultiplets are related. For instance, starting with the field $Y^{i j}$ belonging to a vector supermultiplet, one can generate a linear multiplet upon the following identification,

$$
\begin{aligned}
L^{i j} & \rightarrow 2 Y^{i j}, \\
\varphi^{i} & \rightarrow \mathrm{i} \not \partial \Omega^{i}, \\
E_{\mu} & \rightarrow \partial^{\nu} F_{\nu \mu}, \\
N & \rightarrow \square \sigma,
\end{aligned}
$$

as the reader can easily verify by explicit calculation. At this point one can generate a new vector multiplet, by starting with the field $N$ and identifying it with a new field $\sigma$, etcetera, at the price of including higher and higher powers of derivatives. It is easy to see that the linear multiplet precisely corresponds to the field equations of the vector multiplet. Conversely, the vector multiplet will arise as the field equations of the linearized 
tensor multiplet action in flat space. This relationship is clearly embodied in the density formula that we will define at the end of this section (c.f. (3.10)) and this feature has been exploited extensively in four space-time dimensions, both for $N=1$ and in $N=2$ supersymmetry, but in five dimensions the restrictions are much stronger.

In the superconformal setting the relationship between vector and linear supermultiplets must, however, be modified in view of the additional restrictions posed by superconformal symmetries. For instance, the fields $L^{i j}$ and $Y^{i j}$ behave differently under scale transformations, and, moreover, $L^{i j}$ is invariant under S-supersymmetry, whereas $Y^{i j}$ is not. Nevertheless the relationship can still be established provided one gives up linearity. In the absence of the superconformal background fields in flat space-time, the first component of the correspondence (3.1) is then replaced by (for a single multiplet),

$$
L^{i j} \rightarrow 2 \sigma Y^{i j}+\frac{1}{2} \mathrm{i} \varepsilon^{k(i} \bar{\Omega}_{k} \Omega^{j)}
$$

To establish the existence of this composite linear multiplet one verifies that the lowest component has the correct Weyl weight and is S-supersymmetric, and furthermore, that its supersymmetry variation is expressed in terms of a simple doublet spinor which can then act as the representative of the linear multiplet spinor $\varphi^{i}$. If these criteria are not met, then one will not be dealing with a linear multiplet consisting of $8+8$ degrees of freedom, but with a much larger multiplet. When dealing with several vector multiplets, labeled by indices $I, J, \cdots=1,2, \ldots, n$, the expression (3.2) generalizes only slightly. It remains quadratic on the vector multiplets and depends on it in a symmetric fashion. Hence we start with

$$
L^{i j(I J)}=2 \sigma^{(I} Y^{i j J)}+\frac{1}{2} \mathrm{i} \varepsilon^{k(i} \bar{\Omega}_{k}^{(I} \Omega^{j) J)},
$$

For clarity of the notation, we will henceforth suppress the explicit indices $(I, J)$ on the right-hand side. In the presence of several vector multiplets, $\sigma^{2}$ generalizes to $\sigma^{(I} \sigma^{J)}, \sigma \Omega^{i}$ to $\sigma^{(I} \Omega^{i J)}$, etcetera.

The other components of the corresponding linear multiplet follow by applying successive supersymmetry variations and one finds the following expressions, all manifestly quadratic in the vector multiplet components,

$$
\begin{aligned}
\varphi^{i(I J)}= & \mathrm{i} \sigma \not D \Omega^{i}+\frac{1}{2} \mathrm{i} \not D \sigma \Omega^{i}-8 \sigma^{2} \chi^{i}+Y^{i j} \varepsilon_{j k} \Omega^{k}-\frac{1}{4}\left(\hat{F}_{a b}-6 \sigma T_{a b}\right) \gamma^{a b} \Omega^{i} \\
E^{a(I J)}= & \frac{1}{8} \mathrm{i} \varepsilon^{a b c d e} \hat{F}_{b c} \hat{F}_{d e}+D_{b}\left(\sigma \hat{F}^{b a}-6 \sigma^{2} T^{b a}\right)+\cdots \\
N^{(I J)}= & \frac{1}{2} D^{a} D_{a} \sigma^{2}-\frac{1}{2}\left(D_{a} \sigma\right)^{2}+\left|Y^{i j}\right|^{2} \\
& -\frac{1}{4} \hat{F}_{a b} \hat{F}^{a b}+6 \sigma \hat{F}_{a b} T^{a b}-\sigma^{2}\left(4 D+\frac{39}{2} T^{2}\right)+\cdots
\end{aligned}
$$

where supercovariant terms of higher-order in the fermion fields have been suppressed. It is also possible to derive the expression for the three-rank tensor gauge field associated 
with this multiplet, by requiring (2.18),

$$
\begin{aligned}
E_{\mu \nu \rho}^{(I J)}= & \frac{1}{2} \mathrm{i} e \varepsilon_{\mu \nu \rho \sigma \lambda}\left(\sigma \hat{F}^{\sigma \lambda}-6 \sigma^{2} T^{\sigma \lambda}\right)+\frac{3}{2} W_{[\mu} F_{\nu \rho]}+\frac{1}{4} \bar{\Omega}_{i} \gamma_{\mu \nu \rho} \Omega^{i} \\
& -\frac{3}{2} \mathrm{i} \sigma \bar{\Omega}_{i} \gamma_{[\mu \nu} \psi_{\rho]}{ }^{i}-\frac{3}{4} \sigma^{2} \bar{\psi}_{[\mu i} \gamma_{\nu} \psi_{\rho]}{ }^{i} .
\end{aligned}
$$

The above construction can be generalized to non-abelian vector multiplets as well. In principle, a linear multiplet can also be constructed from hypermultiplets, but the resulting linear multiplet will not be fully realized off-shell.

The same strategy can also be used for constructing a linear multiplet from the square of the Weyl multiplet. In view of the fact that the transformations for the Weyl multiplet fields are not linear, this construction is considerably more complicated than the one above. The starting point, as before, is to define a composite field $L^{\mathrm{W} i j}$ in terms of the Weyl multiplet fields, which satisfies all the requirements for the lowest-dimensional component of a superconformal linear multiplet. This linear multiplet has originally been determined in [13] (with different conventions). In the conventions of this paper we found the following result,

$$
L^{i j \mathrm{~W}}=-\varepsilon^{k(i}\left[\frac{1}{32} \mathrm{i} \bar{R}_{a b k}(Q) R^{j) a b}(Q)+\frac{32}{3} \mathrm{i} \bar{\chi}_{k} \chi^{j)}-\frac{1}{4} T^{a b} R_{a b k}{ }^{j)}(V)\right] .
$$

which, indeed, is S-invariant and transforms under Q-supersymmetry into a spinor doublet. Furthermore it scales with Weyl weight 3, as is appropriate for a linear multiplet. By applying successive supersymmetry transformations, we identify the other components of this linear multiplet,

$$
\begin{aligned}
\varphi^{i \mathrm{~W}}= & \frac{1}{64} R_{a b}{ }^{c d}(M) \gamma_{c d} R^{a b i}(Q)+\frac{1}{32} R_{a b j}{ }^{i}(V) R^{a b j}(Q)-\frac{3}{4} T^{a b} R_{a b}{ }^{i}(S) \\
& -\frac{1}{6} R_{a b j}{ }^{i}(V) \gamma^{a b} \chi^{j}-\frac{3}{8} \mathrm{i} D_{a} T_{b c} \gamma^{c} R^{a b i}(Q)+\frac{3}{16} T^{a b} T_{c d} \gamma^{c d} R_{a b}{ }^{i}(Q) \\
& +4 \mathrm{i} T^{a b} \gamma_{a} D_{b} \chi^{i}-\frac{1}{2} \mathrm{i}\left(\gamma^{a b} \not D T_{a b}+3 \not D \gamma^{a b} T_{a b}\right) \chi^{i}+\frac{8}{3}\left(2 D+3 T^{2}\right) \chi^{i}, \\
\hat{E}^{a \mathrm{~W}}= & -\frac{1}{128} \mathrm{i} \varepsilon^{a b c d e}\left[R_{b c}{ }^{f g}(M) R_{d e f g}(M)+\frac{1}{3} R_{b c j}{ }^{i}(V) R_{d e i}{ }^{j}(V)\right] \\
& +\frac{3}{2} \mathrm{i} \varepsilon^{a b c d e} D_{b}\left[T_{c f} D^{f} T_{d e}+\frac{3}{2} T_{c f} D_{d} T_{e}{ }^{f}\right] \\
& -D_{b}\left[\frac{3}{8} R(M)_{c d}^{a b} T^{c d}+2 T^{a b} D+\frac{3}{4} T^{a b} T^{2}-9 T^{a c} T_{c d} T^{d b}\right]+\cdots, \\
N^{\mathrm{W}}= & \frac{1}{64} R_{a b}{ }^{c d}(M) R_{c d}{ }^{a b}(M)+\frac{1}{96} R_{a b j}{ }^{i}(V) R^{a b}{ }_{i}^{j}(V)+\frac{15}{8} T^{a b} T_{c d} R_{a b}{ }^{c d}(M) \\
& +3 T^{a b} D^{c} D_{a} T_{b c}-\frac{3}{2}\left(D_{a} T_{b c}\right)^{2}+\frac{3}{2} D_{c} T_{a b} D^{a} T^{c b} \\
& -\frac{9}{4} \mathrm{i} \varepsilon_{a b c d e} T^{a b} T^{c d} D_{f} T^{f e}+\frac{8}{3} D^{2}+8 T^{2} D-\frac{33}{8}\left(T^{2}\right)^{2}+\frac{81}{2}\left(T^{a c} T_{b c}\right)^{2} \\
& +\cdots,
\end{aligned}
$$

where the dots refer to fermionic terms, which we will not need for what follows. ${ }^{3}$

\footnotetext{
${ }^{3}$ Note that (3.4) and (3.7) contain second-order superconformally covariant derivatives. For convenience
} 
In order to represent a linear multiplet, the vector $\hat{E}^{a \mathrm{~W}}$ should satisfy the constraint $D_{a} \hat{E}^{a \mathrm{~W}}=0$, as a consequence of which this vector can be expressed in terms of a threerank tensor field $E_{\mu \nu \rho}^{\mathrm{W}}$. In principle, we can determine the full expression of this composite tensor by verifying its supersymmetry transformation (2.18). This is how we originally obtained (3.5). For the Weyl multiplet, however, this calculation is considerably more involved, so that we restrict ourselves to the expression for the purely bosonic terms. The result reads as follows,

$$
\begin{aligned}
E_{\mu \nu \rho}^{\mathrm{W}}= & -\frac{3}{16} \omega_{[\mu}^{a b}\left(\partial_{\nu} \omega_{\rho] a b}-\frac{2}{3} \omega_{\nu a c} \omega_{\rho]}^{c} b\right)-\frac{1}{16} V_{[\mu i}{ }^{j}\left(\partial_{\nu} V_{\rho j}{ }^{i}-\frac{1}{3} V_{\nu j}{ }^{k} V_{\rho] k}{ }^{i}\right) \\
& -9\left(T_{\sigma[\mu} \mathcal{D}^{\sigma} T_{\nu \rho]}+\frac{3}{2} T_{\sigma[\mu} \mathcal{D}_{\nu} T_{\rho]}^{\sigma}\right) \\
& +\mathrm{i} e \varepsilon_{\mu \nu \rho \sigma \lambda}\left(\frac{3}{16} R(M)_{\kappa \tau}{ }^{\sigma \lambda} T^{\kappa \tau}+T^{\sigma \lambda} D+\frac{3}{8} T^{\sigma \lambda} T^{2}-\frac{9}{2} T^{\sigma \kappa} T_{\kappa \tau} T^{\tau \lambda}\right) \\
& +\cdots,
\end{aligned}
$$

where the dots represent the fermionic contributions. It is not difficult to verify that this expression is invariant under scale transformations and conformal boosts, up to tensor gauge transformations and up to terms proportional to fermions (we recall that the spin connection depends both on $b_{\mu}$ and $\psi_{\mu}{ }^{i}$ ), and that the tensor field strength corresponding to it reproduces the bosonic terms in $\hat{E}^{a \mathrm{~W}}$ shown in (3.7).

Finally, we note the existence of a superconformally invariant density for a product of a vector with a tensor supermultiplet. The corresponding expression takes the following form,

$$
\begin{aligned}
e^{-1} \mathcal{L}_{\mathrm{vt}}= & \left(Y_{i j}-\frac{1}{2} \bar{\Omega}_{i} \gamma^{\mu} \psi_{\mu}{ }^{k} \varepsilon_{k j}\right) L^{i j}+\sigma\left(N-\frac{1}{2} \bar{\varphi}_{i} \gamma^{\mu} \psi_{\mu}{ }^{i}\right)+\mathrm{i} \bar{\Omega}_{i} \varphi^{i} \\
& +\frac{1}{6} \mathrm{i} e^{-1} \varepsilon^{\mu \nu \rho \sigma \lambda} W_{\mu} \partial_{\nu} E_{\rho \sigma \lambda}+\frac{1}{4} \mathrm{i} \sigma L^{i j} \bar{\psi}_{\mu i} \gamma^{\mu \nu} \psi_{\nu}{ }^{k} \varepsilon_{k j} .
\end{aligned}
$$

By using the composite linear multiplets defined previously, this density formula thus enables the construction of superconformally invariant actions. This represents a standard way of constructing actions that is also well-known in the context of four space-time dimensions. We will make use of this result in section 5 .

\section{BPS attractor equations}

In this section we derive the conditions for full supersymmetry of the field configuration. Here we follow the systematic approach outlined for four space-time dimensions in [54]. In this section the analysis is done entirely at the off-shell level and we obtain the full we exhibit the bosonic structure of three such expressions,

$$
\begin{aligned}
D_{\mu} D_{a} \sigma & =\mathcal{D}_{\mu} \mathcal{D}_{a} \sigma+2 f_{\mu a} \sigma \\
D_{\mu} D_{a} A_{i}{ }^{\alpha} & =\mathcal{D}_{\mu} \mathcal{D}_{a} A_{i}{ }^{\alpha}+3 f_{\mu a} A_{i}{ }^{\alpha}, \\
D_{\mu} D_{a} T_{c d} & =\mathcal{D}_{\mu} \mathcal{D}_{a} T_{c d}-4 f_{\mu[c} T_{d] a}+4 f_{\mu}{ }^{e} \eta_{a[c} T_{d] e}+2 f_{\mu a} T_{c d} .
\end{aligned}
$$


space-time geometry. Our analysis differs from the one of [17], where on-shell information was already introduced at an earlier stage of the calculation. Only in the next section 5 we will make use of the supersymmetric action. Although our analysis is different in spirit and covers a much larger class of supergravity theories, the results turn out to overlap substantially with those of [8].

\subsection{Supersymmetry}

To analyze supersymmetry one chooses a purely bosonic field configuration and requires that the supersymmetry variation of all fermion fields vanish up to a uniform Ssupersymmetry transformation. In this context it is convenient to define two "compensating' spinor fields, $\zeta_{\mathrm{V}}^{i}$ and $\zeta_{\mathrm{H}}^{i}$, belonging to the vector multiplet sector and the hypermultiplet sector, respectively, which transform linearly under S-supersymmetry,

$$
\zeta_{\mathrm{V}}^{i}=\frac{1}{C(\sigma)} C_{I J K} \sigma^{I} \sigma^{J} \Omega^{i K}, \quad \zeta_{\mathrm{H}}^{i}=-\frac{2}{3 \chi} \varepsilon^{i j} \Omega_{\alpha \beta} A_{j}{ }^{\alpha} \zeta^{\beta} .
$$

Here we have introduced a symmetric three-rank tensor $C_{I J K}$ and a corresponding function $C(\sigma)=C_{I J K} \sigma^{I} \sigma^{J} \sigma^{K}$. The tensor $C_{I J K}$ must be non-vanishing, but other than that there are no immediate restrictions.

It is straightforward to write down the supersymmetry variations of these two spinor fields (which both carry scaling weights equal to $\frac{1}{2}$ ),

$$
\begin{aligned}
\delta \zeta_{\mathrm{V}}^{i} & =\left(T_{a b}-\frac{1}{12} F_{a b}{ }^{I} \partial_{I} \ln C(\sigma)\right) \gamma^{a b} \epsilon^{i}-\frac{1}{6} \mathrm{i} \not \supset \ln C(\sigma) \epsilon^{i}-\frac{1}{3} \varepsilon_{j k} Y^{i j I} \partial_{I} \ln C(\sigma) \epsilon^{k}+\eta^{i}, \\
\delta \zeta_{\mathrm{H}}^{i} & =-\frac{1}{6} \mathrm{i} \not \supset \ln \chi \epsilon^{i}+\frac{1}{3} \mathrm{i} \not \not_{j}{ }^{i} \epsilon^{j}+\eta^{i},
\end{aligned}
$$

where here and henceforth we suppress terms proportional to the fermion fields. Furthermore we made use of the identity [51],

$$
\chi^{-1} \Omega_{\alpha \beta} A_{i}{ }^{\alpha} \mathcal{D}_{\mu} A_{j}{ }^{\beta}=\frac{1}{2} \varepsilon_{i j} \mathcal{D}_{\mu} \ln \chi+k_{\mu i}{ }^{k} \varepsilon_{k j},
$$

where $k_{\mu j}{ }^{i}$ is proportional to the $\mathrm{SU}(2)$ Killing vectors of the underlying hyperkähler cone.

We now require that the S-supersymmetric linear combinations, $\zeta_{\mathrm{V}}^{i}-\zeta_{\mathrm{H}}^{i}, \zeta^{\alpha}-\frac{3}{2} A_{i}{ }^{\alpha} \zeta_{\mathrm{H}}^{i}$, $\Omega^{i I}-\sigma^{I} \zeta_{\mathrm{V}}^{i}, \varphi^{i}-3 \varepsilon_{j k} L^{i j} \zeta_{\mathrm{V}}^{k}$, and $\chi^{i}-\frac{3}{16} T_{a b} \gamma^{a b} \zeta_{\mathrm{V}}^{i}$, do not transform under Q-supersymmetry. This leads to the following conditions,

$$
\begin{array}{rlrl}
\mathcal{D}_{\mu}\left(\chi^{-1 / 2} A_{i}^{\alpha}\right) & =0, & k_{\mu j}{ }^{i} & =0, \\
\partial_{\mu}\left(C^{-1 / 3}(\sigma) \sigma^{I}\right) & =0, & R_{\mu \nu i}{ }^{j}(V) & =0, \\
\mathcal{D}_{\mu}\left(C^{-1}(\sigma) L^{i j}\right) & =0, & Y^{i j I} & =0, \\
C(\sigma) \chi^{-1} & =\text { constant }, & N & =0, \\
F_{a b} & =4 \sigma^{I} T_{a b}, & \hat{E}^{a} & =0, \\
\mathcal{D}_{[a}\left(C^{1 / 3}(\sigma) T_{b c]}\right) & =0, & D & =0, \\
\mathcal{D}_{b}\left(C^{2 / 3}(\sigma) T^{b a}\right) & =\mathrm{i} \varepsilon^{a b c d e} T_{b c} T_{d e} C^{2 / 3}(\sigma), &
\end{array}
$$

which were also given in [17] in the conventions of [13, 49]. However, there are further constraints in view of the fact that all fermionic quantities must vanish under supersymmetry. 
Experience from the corresponding analysis in four space-time dimensions [54] indicates that one must also consider the variations of $R_{a b}{ }^{i}(Q)-\left(T_{c d} \gamma_{c d} \gamma_{a b}-4 T_{a b}\right) \zeta_{\mathrm{V}}^{i}$, and of $D_{\mu} \zeta_{\mathrm{H}}$. Combining the result of the first variation with the previous results, one finds,

$$
\begin{aligned}
\mathcal{D}_{c} T_{a b}= & \frac{1}{2} \mathrm{i} \eta_{c[a} \varepsilon_{b] d e f g} T^{d e} T^{f g} \\
& -\frac{1}{3}\left[2 \mathcal{D}_{[a} \ln C(\sigma) T_{b] c}-\mathcal{D}_{c} \ln C(\sigma) T_{a b}-2 \mathcal{D}^{d} \ln C(\sigma) T_{d[a} \eta_{b] c}\right], \\
R_{a b}{ }^{c d}(M)= & \left.-2\left[T^{2} \delta_{a b}^{c d}+4 T_{a b} T^{c d}+4 T_{[a}^{c} T_{b]}^{d}-8 T_{e[a} T^{e[c} \delta_{b]}{ }^{d}\right]\right] .
\end{aligned}
$$

In addition one considers the variation of the S-invariant combination, $D_{\mu} \zeta_{\mathrm{H}}^{i}-$ $\frac{1}{6}\left[\delta_{j}^{i} \not D \ln \chi \gamma_{\mu}-2 \hat{\not}_{j}{ }^{i} \gamma_{\mu}-6 \mathrm{i} T_{\mu a} \gamma^{a}\right] \zeta_{\mathrm{H}}^{j}$, subject to the conditions (4.4). This confirms the consistency of the previous results and, in addition, gives rise to one more condition,

$$
f_{\mu}{ }^{a}=-\frac{1}{6} \mathcal{D}_{\mu} \mathcal{D}^{a} \ln \chi+\frac{1}{18} \mathcal{D}_{\mu} \ln \chi \mathcal{D}^{a} \ln \chi-4 T_{\mu b} T^{a b}+\frac{1}{4}\left[3 T_{b c} T^{b c}-\frac{1}{9}\left(\mathcal{D}_{b} \ln \chi\right)^{2}\right] e_{\mu}{ }^{a} .
$$

Using the arguments presented in [54], we conclude that the above equations (4.4), (4.5) and (4.6) comprise all the conditions for a supersymmetric field configuration consisting of the Weyl multiplet, vector multiplets, linear multiplets and hypermultiplets, without imposing equations of motion. Because the fermionic equations of motion must be satisfied, simply because of supersymmetry, most of the bosonic equations of motion must be satisfied as well. There are, however, exceptions, such as the equation of motion associated with the scalar field $D$ belonging to the Weyl multiplet, which does not appear as the supersymmetry variation of a fermionic expression.

Combining the second equation of (4.5) with (4.6), we derive the following equation,

$$
\begin{aligned}
\mathcal{R}_{a b}{ }^{c d}(\omega, e)= & 2 e_{a}{ }^{\mu} e_{b}{ }^{\nu}\left(\partial_{[\mu} \omega_{\nu]}{ }^{c d}-\omega_{[\mu}{ }^{c e} \omega_{\nu] e}{ }^{d}\right) \\
= & -8\left(T_{a b} T^{c d}+T_{a}{ }^{[c} T_{b}{ }^{d]}\right)+\delta_{[a}^{c} \delta_{b]}^{d}\left(4 T_{e f} T^{e f}-\frac{2}{9}\left(\mathcal{D}_{e} \ln \chi\right)^{2}\right) \\
& -\delta_{[a}{ }^{[c}\left(16 T_{b] e} T^{d] e}+\frac{4}{3} \mathcal{D}_{b]} \mathcal{D}^{d]} \ln \chi-\frac{4}{9} \mathcal{D}_{b]} \ln \chi \mathcal{D}^{d]} \ln \chi\right) .
\end{aligned}
$$

\subsection{Space-time geometry}

Before discussing the resulting space-time geometry we have to impose a number of gauge choices. We set the dilatational gauge field $b_{\mu}=0$ (in fact, K-invariance implies that the equations found above are already independent of $b_{\mu}$ ) and furthermore we set the function $C(\sigma)$ equal to a constant $C$. This implies that also $\chi$ becomes a constant. The ratio of the two constants $C$ and $\chi$ will eventually be defined by the equation of motion for the field $D$, but at the moment we proceed without making reference to any particular Lagrangian. Note that the various fields will still be subject to constant scale transformations which are a remnant of the full space-time dependent dilatations. Physical results should, of course, be insensitive to these scale transformations. In addition we set the $\mathrm{SU}(2)$ gauge connections to zero, in view of the fact that their field strength is vanishing (c.f. (4.4)). In this situation the various scalar fields $\sigma^{I}$ and $L^{i j}$ are all constant. 
The resulting geometry is now of a special type, as the tensor $T_{\mu \nu}$ is an example of a conformal Killing-Yano tensor [55]. Locally, in five space-time dimensions, this tensor generically induces a family of pairs of two-surfaces which together with the fifth orthogonal dimension foliate the space-time. It also leads to a Killing vector associated with this fifth dimension and a symmetric Killing tensor,

$$
\xi^{\mu}=\mathrm{i} e^{-1} \varepsilon^{\mu \nu \rho \sigma \tau} T_{\nu \rho} T_{\sigma \tau}, \quad K_{\mu \nu}=T_{\mu \rho} T_{\nu}{ }^{\rho},
$$

where $e=\operatorname{det}\left(e_{\mu}{ }^{a}\right)$. Using the properties of the tensor $T_{\mu \nu}$ (in the gauge indicated above), we obtain the following results for the Riemann tensor and for the derivative of $T_{\mu \nu}$,

$$
\begin{aligned}
\mathcal{R}_{a b}{ }^{c d} & =-8\left(T_{a b} T^{c d}+T_{a}{ }^{[c} T_{b}{ }^{d]}\right)-16 \delta_{[a}^{[c} T_{b] e} T^{d] e}+4 \delta_{[a}^{c} \delta_{b]}^{d} T_{e f} T^{e f}, \\
\mathcal{D}_{\rho} T_{\mu \nu} & =\frac{1}{2} g_{\rho[\mu} \xi_{\nu]} .
\end{aligned}
$$

Furthermore we note the results,

$$
\begin{aligned}
\mathcal{D}_{\mu} \xi_{\nu} & =-\mathrm{i} e \varepsilon_{\mu \nu \rho \sigma \tau} \xi^{\rho} T^{\sigma \tau}, & \xi^{\mu} T_{\mu \nu} & =0, \\
\mathcal{D}_{\rho} K_{\mu \nu} & =-\frac{1}{2} \xi_{(\mu} T_{\nu) \rho}, & T^{2} & \equiv\left(T_{a b}\right)^{2}=\text { constant }
\end{aligned}
$$

From these equations it is clear that $\xi^{\mu}$ is indeed a Killing vector. Furthermore one may easily verify that the Riemann tensor satisfies the Bianchi identity.

If $\xi^{\mu}$ vanishes then the tensors $T_{\mu \nu}$ and $K_{\mu \nu}$ are covariantly constant and so is the Riemann tensor so that we are dealing with a locally symmetric space. In this particular case the space is in fact the product of a two- and a three-dimensional maximally symmetric space, as the Riemann tensor decomposes into two Riemann tensors corresponding to these subspaces satisfying $R_{\hat{a} \hat{b}} \hat{c} \hat{d} \propto c T^{2} \delta_{[\hat{a}}^{\hat{c}} \delta_{\hat{b}]}^{\hat{d}}$, with proportionality $c=-16$ and $c=4$ for the two- and the three-dimensional subspace, respectively. Here the indices $\hat{a}, \hat{b}, \hat{c}, \hat{d}$ refer to the tangent-space projected onto the two- or three-dimensional subspaces.

Rather than considering this case any further, we concentrate on the more general case where $\xi^{\mu} \neq 0$ and return to the limit of vanishing $\xi^{\mu}$ at the end. Obviously the line element must reflect the isometry associated with the Killing vector $\xi^{\mu}$. Choosing a coordinate $\psi$ by $\xi^{\mu} \partial_{\mu}=\partial / \partial \psi$, we decompose the coordinates into $\psi$ and four-dimensional coordinates $x^{m}$, where $m=1,2,3,4$, without committing ourselves to a certain signature yet. ${ }^{4}$ Correspondingly, the tangent-space indices $a=1,2, \ldots, 5$ are decomposed into $a=5$ and indices $p, q, \ldots=1,2,3,4$. Upon a suitable local Lorentz transformation, the fünfbein is brought into the form,

$$
e_{\mu}^{5} \mathrm{~d} x^{\mu}=\mathrm{e}^{g}\left[\mathrm{~d} \psi+\sigma_{m} \mathrm{~d} x^{m}\right], \quad e_{\mu}^{p} \mathrm{~d} x^{\mu}=\mathrm{e}^{-g / 2} \hat{e}_{m}^{p} \mathrm{~d} x^{m} .
$$

In view of the isometry corresponding to shifts of the coordinate $\psi$ we may assume that $g, \sigma_{m}$ and the vierbein field $\hat{e}_{m}^{p}$ do not depend on $\psi$. The corresponding inverse fünfbein

\footnotetext{
${ }^{4}$ At this point we are using Pauli-Källén metric conventions, where the signature is determined by making one of the coordinates purely imaginary. This enables us to consider all possible signatures at once, so that this analysis encompasses the solutions for minimal supergravity found in [8]. Momentarily we will assume that the Killing vector $\xi^{\mu}$ is spacelike.
} 
components are given by,

$$
e_{5}{ }^{\psi}=\mathrm{e}^{-g}, \quad e_{5}{ }^{m}=0, \quad e_{p}{ }^{\psi}=-\sigma_{p} \mathrm{e}^{g / 2}, \quad e_{p}{ }^{m}=\mathrm{e}^{g / 2} \hat{e}_{p}{ }^{m},
$$

where, on the right-hand side, four-dimensional tangent-space and world indices are converted by the vierbein $\hat{e}_{m}{ }^{p}$ and its inverse (so that, e.g. $\sigma_{p}=\hat{e}_{p}{ }^{m} \sigma_{m}$, and the covariant derivative $\nabla_{p}$ contains the spin connection $\hat{\omega}_{m}^{p q}$, associated with the vierbein $\hat{e}_{m}^{p}$ ). This leads to the following expressions for the spin connection, $\omega_{a b c} \equiv e_{a}{ }^{\mu} \omega_{\mu c d}$,

$$
\begin{aligned}
& \omega_{p q r}=\mathrm{e}^{g / 2}\left[\hat{\omega}_{p q r}+\delta_{p[q} \nabla_{r]} g\right], \\
& \omega_{5 p q}=\omega_{q p 5}=\frac{1}{2} \mathrm{e}^{2 g} \mathcal{Q}_{p q}, \\
& \omega_{55 p}=-\mathrm{e}^{g / 2} \nabla_{p} g,
\end{aligned}
$$

where $\mathcal{Q}_{p g}$ equals,

$$
\mathcal{Q}_{p q}=\hat{e}_{p}{ }^{m} \hat{e}_{q}{ }^{n} \mathcal{Q}_{m n}, \quad \mathcal{Q}_{m n}=\partial_{m} \sigma_{n}-\partial_{n} \sigma_{m} .
$$

Let us now return to (4.9) and consider the second equation, which we write in tangentspace indices as,

$$
e_{c}{ }^{\mu} \partial_{\mu} T_{a b}+2 \omega_{c[a}{ }^{d} T_{b] d}=\frac{1}{2} \delta_{c[a} \delta_{b] 5} \xi,
$$

where we made use of the fact that $T_{5 a}=0$ and defined $\xi=\mathrm{i} \varepsilon^{p q r s} T_{p q} T_{r s}$, where $\varepsilon^{p q r s}=$ $\varepsilon^{5 p q r s}$ so that $\xi^{\psi}=\mathrm{e}^{-g} \xi$. Changing the overall sign of the epsilon tensor is irrelevant as it only corresponds to a sign change of the coordinate $\psi{ }^{5}$ Imposing the equations contained in (4.15) leads to the following results,

$$
\partial_{\psi} T_{a b}=0, \quad \mathcal{Q}_{p q}=-2 \mathrm{ie}^{-2 g} \varepsilon_{p q r s} T^{r s}, \quad \nabla_{p} T_{q r}=0, \quad g=\text { constant } .
$$

These results are consistent with what is found when considering the Riemann tensor from the connections (4.13) upon comparison with the first equation (4.9). ${ }^{6}$ Here and henceforth we will be assuming that the four-dimensional subspace has signature $(-,+,+,+)$, so that the Killing vector $\xi^{\mu}$ is spacelike and $\xi$ is real. The various curvature components read,

$$
\begin{aligned}
\mathcal{R}_{p q 5 r}= & -\frac{1}{2} \mathrm{e}^{5 g / 2}\left[\nabla_{r} \mathcal{Q}_{p q}+\nabla_{r} g \mathcal{Q}_{p q}+\nabla_{[p} g \mathcal{Q}_{q] r}-\delta_{r[p} \mathcal{Q}_{q] s} \nabla^{s} g\right] \\
\mathcal{R}_{5 p 5 q}= & \mathrm{e}^{g}\left[\nabla_{p} \nabla_{q} g-\frac{1}{2} \delta_{p q}\left(\nabla_{r} g\right)^{2}+2 \nabla_{p} g \nabla_{q} g\right]-\frac{1}{4} \mathrm{e}^{4 g} \mathcal{Q}_{p r} \mathcal{Q}_{q}{ }^{r} \\
\mathcal{R}_{p q r s}= & \mathrm{e}^{g} \mathcal{R}_{p q r s}(\hat{\omega})-2 \mathrm{e}^{g} \delta_{[p[r}\left[\nabla_{s]} \nabla_{q]} g+\frac{1}{2} \nabla_{s]} g \nabla_{q]} g-\frac{1}{4} \delta_{s] q]}\left(\nabla_{u} g\right)^{2}\right] \\
& +\frac{1}{2} \mathrm{e}^{4 g}\left[\mathcal{Q}_{p q} \mathcal{Q}_{r s}-\mathcal{Q}_{p[r} \mathcal{Q}_{s] q}\right]
\end{aligned}
$$

where the right-hand side is consistently written in four-dimensional notation. Obviously $\mathcal{R}_{p q 5 r}$ must vanish in order to be consistent with the first equation (4.9), and this is indeed

\footnotetext{
${ }^{5}$ In Pauli-Källén notation we now fix convention such that $\varepsilon_{\mu \nu \rho \sigma \tau} x^{\mu} x^{\nu} x^{\rho} x^{\sigma} x^{\tau}=\mathrm{i} 5 ! x^{0} x^{1} x^{2} x^{3} x^{5}$.

${ }^{6}$ We note that (4.16) has been derived from (4.15) assuming $\operatorname{det}[T] \neq 0$. For $\operatorname{det}[T]=0$ one can arrive at the same result by also making use of (4.9) and (4.17).
} 
what is implied by the earlier results (4.16). Likewise the expression for $\mathcal{R}_{5 p 5 q}$ is consistent with the corresponding equation (4.9). Hence we are left to analyse the last equation of (4.17), which determines the four-dimensional Riemann tensor $\mathcal{R}(\hat{\omega})$ according to

$$
\mathcal{R}_{p q r s}(\hat{\omega})=-16 \mathrm{e}^{-g}\left[4 \delta_{[p[r} T_{s] t} T_{q]}^{t}-\delta_{p[r} \delta_{s] q} T^{2}\right] .
$$

The Ricci scalar, $\mathcal{R}_{a b}{ }^{a b}(\hat{\omega})=0$. Further inspection shows that this Riemann tensor corresponds to a product of two two-dimensional spaces with equal radii, namely $A d S_{2}$ and $S^{2}$. The geometry thus takes the form of a circle (parametrized by the coordinate $\psi$ ) non-trivially fibered over an $A d S_{2} \times S^{2}$ base space. We now adopt four-dimensional coordinates by writing the respective metrics in the standard form of a Bertotti-Robinson and a two-sphere metric, with coordinates $t, r$, and $\theta, \varphi$, respectively, so that the fivedimensional line element takes the following form ( $r$ is non-negative and $\theta$ and $\varphi$ have periodicity $\pi$ and $2 \pi$, respectively),

$$
\begin{aligned}
d s^{2} & =\frac{1}{16 v^{2}}\left(-r^{2} \mathrm{~d} t^{2}+\frac{\mathrm{d} r^{2}}{r^{2}}+\mathrm{d} \theta^{2}+\sin ^{2} \theta \mathrm{d} \varphi^{2}\right)+\mathrm{e}^{2 g}(\mathrm{~d} \psi+\sigma)^{2}, \\
\sigma & =-\frac{1}{4 v^{2}} \mathrm{e}^{-g}\left(T_{23} r \mathrm{~d} t-T_{01} \cos \theta \mathrm{d} \varphi\right),
\end{aligned}
$$

corresponding to,

$$
Q_{t r}=\frac{1}{4 v^{2}} \mathrm{e}^{-g} T_{23}, \quad Q_{\theta \varphi}=-\frac{1}{4 v^{2}} \mathrm{e}^{-g} T_{01} \sin \theta .
$$

Here and henceforth we use the definition,

$$
v=\sqrt{\left(T_{01}\right)^{2}+\left(T_{23}\right)^{2}}
$$

where $T_{01}$ and $T_{23}$ are the nonvanishing components of the tensor field $T_{a b}$, where the local Lorentz indices are $(0,1,2,3)$. Note that the vierbein fields can be chosen diagonally; their values can be read off from (4.19),

$$
e_{m}^{p} \mathrm{~d} x^{m}=\frac{1}{4 v}\left(r \mathrm{~d} t, \frac{\mathrm{d} r}{r}, \mathrm{~d} \theta, \sin \theta \mathrm{d} \varphi\right), \quad(p=0,1,2,3) .
$$

In this Lorentz frame, the fields $T_{a b}$ are constant. For future use we also list the nonvanishing spin-connection fields,

$$
\begin{aligned}
\omega_{m}^{p q} & =\stackrel{\circ}{\omega}_{m}^{p q}+\frac{1}{2} \sigma_{m} \mathrm{e}^{3 g} \mathcal{Q}^{p q}, \\
\omega_{m}{ }^{p 5} & =\frac{1}{2} e_{m q} \mathrm{e}^{2 g} \mathcal{Q}^{p q}, \\
\omega_{\psi}{ }^{p q} & =\frac{1}{2} \mathrm{e}^{3 g} \mathcal{Q}^{p q},
\end{aligned}
$$

where $\stackrel{\circ}{\omega}_{t} 01=-r$ and $\stackrel{\circ}{\omega}_{\varphi}{ }^{23}=\cos \theta$. 
Observe that $\sigma^{I}, T_{a b}, v$ and $\mathrm{e}^{-g}$ transform with weight +1 under the (constant) scale transformations inherited from the five-dimensional dilatations. As a result, the metric (4.19) scales uniformly with weight -2 and the one-form $\sigma$ is inert under scale transformations. Note that $\sigma$ is determined up to a four-dimensional gauge transformation associated with shifts of the coordinate $\psi$ with a function depending on the four-dimensional coordinates. Such diffeomorphisms leave the form of the line element invariant.

Let us now further discuss the line element (4.19). Assuming that $T_{01} \neq 0$, we can rewrite the line element in the form,

$$
\begin{aligned}
d s^{2}= & -\frac{\rho^{4}}{16 v^{2}}\left(\frac{T_{01}}{v} \mathrm{~d} t+\frac{T_{23}}{v \rho^{2}}\left(\cos \theta \mathrm{d} \varphi+\frac{1}{p^{0}} \mathrm{~d} \psi\right)\right)^{2} \\
& +\frac{1}{4 v^{2} \rho^{2}}\left(\mathrm{~d} \rho^{2}+\frac{\rho^{2}}{4}\left(\mathrm{~d} \theta^{2}+\mathrm{d} \varphi^{2}+\frac{1}{\left(p^{0}\right)^{2}} \mathrm{~d} \psi^{2}+\frac{2}{p^{0}} \cos \theta \mathrm{d} \varphi \mathrm{d} \psi\right)\right),
\end{aligned}
$$

where we used the definitions

$$
\rho=\sqrt{r}, \quad p^{0}=\frac{\mathrm{e}^{-g}}{4 v^{2}} T_{01} .
$$

To make $p^{0}$ unambiguous we fix the periodicity interval for $\psi$ to $4 \pi$. The second term of the line element then corresponds to a flat metric, up to an overall warp factor $(2 v \rho)^{-2}$. To see this we combine the four Cartesian coordinates into two complex ones, which we parametrize as,

$$
z_{1}=\rho \cos \theta / 2 \exp \frac{1}{2} \mathrm{i}\left[\psi / p^{0}+\varphi\right], \quad z_{2}=\rho \sin \theta / 2 \exp \frac{1}{2} \mathrm{i}\left[\psi / p^{0}-\varphi\right] .
$$

Clearly for $\left|p^{0}\right|=1$ we cover the whole four-dimensional space $\mathbb{R}^{4}$. For $\left|p^{0}\right| \neq 1$ we have a conical singularity at the origin. In all cases the three-dimensional horizon is located at $r=0$ and its cross-sectional area is equal to

$$
A_{3}=\int_{\Sigma_{\mathrm{hor}}}=\pi^{2} v^{-2} \mathrm{e}^{g} .
$$

Observe that this result is not invariant under the scale transformations introduced earlier, which simply reflects the fact that the line element is not invariant either. Furthermore the bi-normal tensor at the horizon is the same in all cases when given with tangent space indices. Its only non-vanishing components are,

$$
\varepsilon_{01}= \pm 1
$$

so that $\varepsilon_{\mu \nu} \varepsilon^{\mu \nu}=-2$. Both (4.27) and (4.28) can be derived by first determining the binormal tensor and the cross-sectional area in a coordinate frame that is non-singular at the horizon, and subsequently converting the results to the singular frame used in the text.

The line element (4.24) describes the near-horizon geometry of the spinning charged black hole [5] (see also, [6]), and we observe that the rotation is associated with a globally defined one-form on $S^{3}$, in view of $\operatorname{Im}\left[z_{1} \mathrm{~d} z_{1}{ }^{*}+z_{2} \mathrm{~d} z_{2}{ }^{*}\right]=\rho^{2}\left[\left(p^{0}\right)^{-1} \mathrm{~d} \psi+\cos \theta \mathrm{d} \varphi\right]$. Clearly 
the angular momentum of the black hole is proportional to $T_{23}$. When $T_{23}=0$ we are dealing with a static black hole and the near-horizon geometry is given by,

$$
d s^{2}=\frac{1}{16 v^{2}}\left(-r^{2} \mathrm{~d} t^{2}+\frac{\mathrm{d} r^{2}}{r^{2}}\right)+\frac{1}{4 v^{2}} d s^{2}\left(S^{3} / \mathbb{Z}_{p^{0}}\right) .
$$

Finally we turn to the case $T_{01}=0$ where we find,

$$
d s^{2}=\frac{1}{16 T_{23}^{2}} \frac{\mathrm{d} r^{2}}{r^{2}}+\mathrm{e}^{2 g} \mathrm{~d} \psi^{2}-\frac{\mathrm{e}^{g}}{2 T_{23}} r \mathrm{~d} \psi \mathrm{d} t+\frac{1}{16 T_{23}{ }^{2}} \mathrm{~d} s^{2}\left(S^{2}\right),
$$

where $\mathrm{d} s^{2}\left(S^{2}\right)$ is the line element belonging to the unit two-sphere. The first three terms constitute a metric which is locally $A d S_{3}$ so that the near-horizon geometry is that of $A d S_{3} \times S^{2}$. This is the near-horizon geometry of a supersymmetric black ring, or, when we drop the identification $\psi \cong \psi+4 \pi$, of an infinitely long black string.

\subsection{Gauge fields}

According to (4.4), the field strengths $F_{\mu \nu}{ }^{I}$ are determined in terms of the tensor field $T_{a b}$,

$$
F_{t r}^{I}=\frac{\sigma^{I}}{4 v^{2}} T_{01}, \quad F_{\theta \varphi}^{I}=\frac{\sigma^{I}}{4 v^{2}} T_{23} \sin \theta .
$$

At this point we can define magnetic charges associated with $Q_{\theta \varphi}$ and $F_{\theta \varphi}{ }^{I}$. Employing the same conventions for these field strengths (apart from a relative sign between $p^{0}$ and $\left.p^{I}\right)$, we define

$$
p^{0}=\frac{\mathrm{e}^{-g}}{4 v^{2}} T_{01}, \quad p^{I}=\frac{\sigma^{I}}{4 v^{2}} T_{23},
$$

with the same expression for $p^{0}$ as given in (4.25). In the five-dimensional context, the $p^{I}$ will play the role of dipole magnetic charges. They are proportional to $T_{23}$, so they will vanish for a static black hole. The definition of the electric charges, which involves the equations of motion, will be discussed in later sections. From (4.31) we can determine the vector potentials,

$$
W_{\mu}^{I}(x) \mathrm{d} x^{\mu}=-\frac{\sigma^{I}}{4 v^{2}}\left(T_{01} r \mathrm{~d} t+T_{23} \cos \theta \mathrm{d} \varphi\right)+\mathrm{d} \Lambda^{I}(x),
$$

up to an abelian gauge transformation, parametrized by $\Lambda^{I}(x)$.

For the spinning black hole, where $T_{01} \neq 0$, the gauge transformation can be chosen such that the gauge potentials are globally defined on $S^{3}$. To see this one makes use of the observation preceding (4.29) in the previous subsection, which leads to,

$$
W_{\mu}^{I} \mathrm{~d} x^{\mu}=-\frac{\sigma^{I}}{4 v^{2}}\left(T_{01} r \mathrm{~d} t+T_{23}\left(\frac{\mathrm{d} \psi}{p^{0}}+\cos \theta \mathrm{d} \varphi\right)\right) .
$$

In the case of the black ring, where $T_{01}=0$, the gauge transformations in (4.33) introduce an uncontractible component corresponding to Wilson lines around the circle parametrized by $\psi$. The proper definition of the Wilson line moduli is subtle due to the presence of the charges $p^{I}$ and the $S^{1} \times S^{2}$ topology, as we shall discuss below. In any case, 
due to the presence of large gauge transformations (i.e. gauge transformations that cannot be connected continuously to the identity), these moduli $a^{I}$ should be periodically identified and furthermore they should be defined such that they are not subject to small gauge transformations. At any rate the gauge fields are expected to contain the following terms,

$$
W_{\mu}^{I} \mathrm{~d} x^{\mu}=-p^{I} \cos \theta \mathrm{d} \varphi+a^{I} \mathrm{~d} \psi .
$$

However, unlike in the case of the spinning black holes, the gauge fields are not globally defined, as is obvious from the fact that the monopole fields are sourced by Dirac strings. This phenomenon implies that the gauge fields should be defined in patches, connected by suitable gauge transformations. In the context of five space-time dimensions the Dirac strings are degenerate and one is actually dealing with Dirac membranes. Just as in the case of Dirac strings, the Dirac membranes are subject to constraints, some of them related to charge quantization (to appreciate this, the reader may consult [56, 57], where some of this is explained in the context of $2+1$ dimensions).

For a single black ring and for multiple concentric black rings, the appropriate sections have been considered in [12], guided by the explicit ring solutions [7] and [9]. Although these results were obtained without taking into account possible higher-derivative interactions, they should still apply to the general case, as the choice of the sections and the corresponding Dirac membranes is entirely based on the topology of the underlying charge configuration. With this in mind we replace (4.35) by the following sections (for a single ring),

$$
W_{\mu}^{I} \mathrm{~d} x^{\mu}=-p^{I}\left[\cos \theta \mathrm{d} \varphi \pm \mathrm{d}\left(\varphi+\frac{1}{2} \psi\right)\right]+a^{I} \mathrm{~d} \psi,
$$

where we note that $\cos \theta$ can be extended globally into the ring coordinate conventionally denoted by $x[7,58]$. For $x=1$ and $x=-1$ one is dealing with the inner and the outer part, respectively, of the two-dimensional plane that contains the ring. Hence the plus sign in (4.36) refers to the section that is singularity free in the outer part of plane, and the minus sign to the section that is singularity free in the inner part.

The nontrivial, and somewhat unexpected, feature of (4.36), is that the gauge transformation between the two patches involves a $\mathrm{d} \psi$ component, contrary to what one would expect based on intuition from four dimensions. Indeed, in the case of an infinite black string, this gauge transformation is just $\propto p^{I} \mathrm{~d} \varphi$. However, the ring topology requires a more extended gauge transformation.

One way to understand this difference is to appreciate the fact that, in order that the Dirac membrane be unobservable, the gauge transformation between the patches must allow for general deformations of its worldvolume irrespective of its topology. Choosing a topologically trivial brane on each patch, say along the north and south pole of each sphere on the ring (see the two figures on the left-hand side of figure 1), leads to the gauge transformation $-2 p^{I} \mathrm{~d} \varphi$ between the patches. This is also the only possible choice for an infinite string. But in the case of a proper ring embedded in a four dimensional space, ${ }^{7}$ the topology of the spatial manifold $\mathcal{M}^{4}$ corresponding to the embedding space minus the ring

\footnotetext{
${ }^{7}$ We assume a topologically trivial embedding space, like $\mathbb{R}^{4}$ or Taub-NUT, in the following discussion.
} 

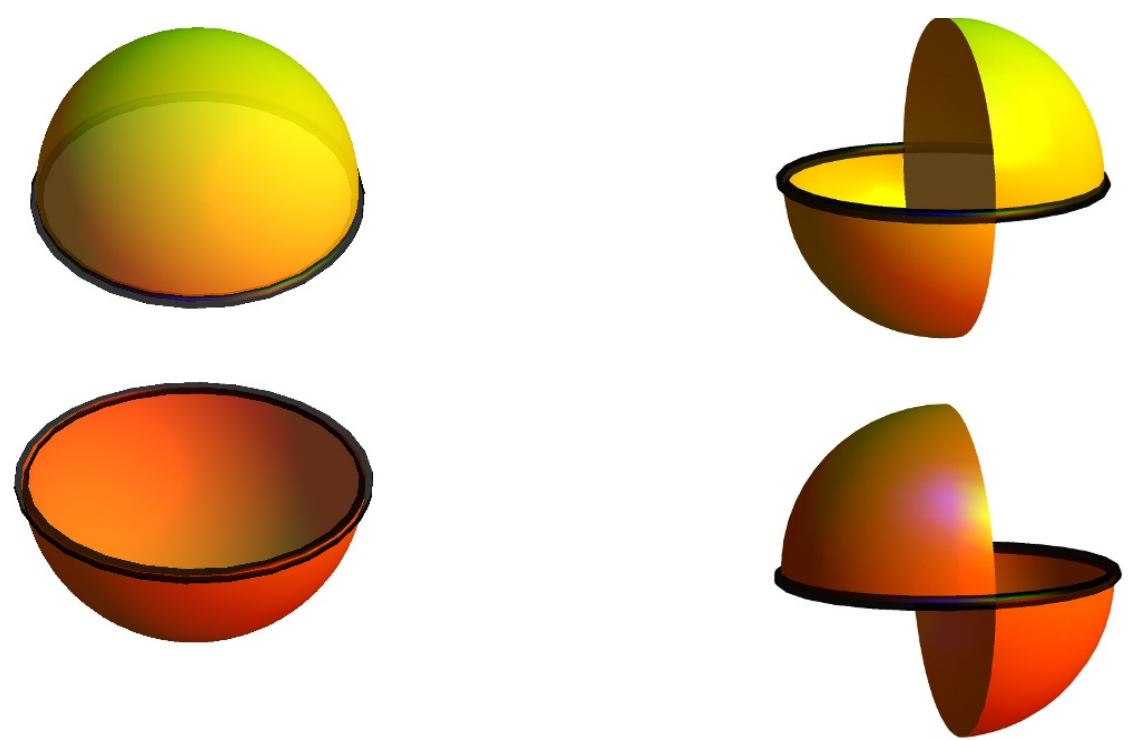

Figure 1. The two figures on the left-hand side correspond to the two different gauge field patches based on a topologically trivial choice for the two-dimensional Dirac brane. The three-dimensional hypersurface bounded by the two branes is a ball $B^{3}$. The gauge transformation associated with the transition between the two patches has only components along the angle $\varphi$ not shown in the picture. On the right-hand side the two figures show a singular limit of the relevant but non-trivial choice for the Dirac brane. The three-dimensional hypersurface connecting the two branes is the sum of the $B^{3}$ above and the generator of $H_{3}\left(\mathcal{M}^{4}\right)$. The corresponding gauge transformation has an additional component along the ring circle, normal to the plane.

is nontrivial. Possible Dirac branes are classified as the boundaries of three-dimensional spatial hypersurfaces. Thus it is important to know the third homology group $H_{3}\left(\mathcal{M}^{4}\right)$, since the Dirac brane can also be the boundary of a non-trivial hypersurface, as opposed to the trivial one discussed above.

In the case at hand it can be shown that $H_{3}\left(\mathcal{M}^{4}\right)=\mathbb{Z}$, so that the generator of the group is a hypersurface with no boundary that wraps the ring once. A corresponding Dirac brane is described as the boundary of the sum of the topologically trivial hypersurface and this generator. Such a brane starts at the north pole of the sphere at some point along the ring. When moving along the $S^{1}$ of the ring, this brane rotates to the south pole and subsequently it returns to the north pole when reaching the point of departure. A singular limit of this surface is shown on the right-hand side of figure 1. Using the construction based on de Rham currents in [56, 57, 59], this leads to a gauge transformation between the gauge field patches that is proportional to the Poincaré dual of the generator described above. A component along $\psi$ is obviously necessary due to the plane in the centre. The relative coefficient in the gauge transformation $\mathrm{d}\left(\varphi+\frac{1}{2} \psi\right)$ has been fixed by demanding periodicity of this generator. Finally, note that higher wrappings would introduce integral multiples of the same one-form, and are therefore irrelevant in view of the integral shift symmetry of $a^{I}$.

The way to measure the Wilson line moduli $a^{I}$ now proceeds through the Chern-Simons 
charges of the ring, defined by the integral over the 3-cycle associated with the horizon $\Sigma$,

$$
Q_{I}^{\mathrm{CS}} \propto \oint_{\Sigma} C_{I J K} W^{J} \wedge F^{K}
$$

It was demonstrated in [12], by using the sections (4.36) and carefully evaluating the integral, that the Chern-Simons charges are linearly related to the moduli $a^{I}$, i.e., $Q_{I}^{\mathrm{CS}} \propto$ $C_{I J K} a^{J} p^{K}$. The use of the sections (4.36) is essential for obtaining this relationship, so that the $a^{I}$, which are identified in this way, are no longer subject to small gauge transformations. Here it is relevant that the Chern-Simons charges are also invariant under small gauge transformations. This result is also consistent with large gauge transformations as both the $a^{I}$ and the $Q_{I}^{\mathrm{CS}}$ change under a large gauge transformations by an integer (in proper units).

Although it is not the primary purpose of this paper to consider multi-ring solutions, it is illuminating to briefly consider the situation of concentric rings [9]. Labeling the rings by an index $i$, one introduces the moduli $a^{I}{ }_{i}$ and the charges $p^{I}{ }_{i}$ of the $i$-th ring. Following the same logic as above, an extended set of sections generalizing (4.36) can be found that matches the one used in [12]. One can then derive the following relation,

$$
Q_{I}^{\mathrm{CS}} \propto C_{I J K}\left[\sum_{i}\left(2 a^{J}+p^{J}\right)_{i} p^{K}{ }_{i}-\left(\sum_{i} p^{J}{ }_{i}\right)\left(\sum_{j} p^{K}{ }_{j}\right)\right]
$$

which, for a single ring, reduces to the previous result. The above relation indicates that the Chern-Simons charges are not additive, unlike the moduli $\left(a^{I}\right)_{i}$ and the charges $\left(p^{I}\right)_{i}$ associated with the various rings. In fact, as we will establish later in section 8 , the best way to write this result is as follows,

$$
Q_{I}^{\mathrm{CS}}-6 C_{I J K} P^{J} P^{K}=-12 C_{I J K} \sum_{i}\left(a^{J}+\frac{1}{2} p^{J}\right)_{i} p^{K}{ }_{i},
$$

where $P^{I}{ }_{i}=\sum_{i} p_{i}{ }_{i}$. This indicates that the expression on the left-hand side is in fact additive. We will return to this topic in section 8 .

\subsection{Linear multiplets}

As a last topic of this section we reconsider the two linear multiplets constructed in section 3 from the product of two vector multiplets and from the square of the Weyl multiplet both vanish for BPS configurations, as the reader can easily verify. However, the corresponding three-forms, denoted by $E_{\mu \nu \rho}$, do not necessarily vanish. Since these quantities will play a role in what follows, we will evaluate some of the corresponding expressions here.

First of all, we present some components of the tensor field $E_{\mu \nu \rho}^{(I J)}$, defined in (3.5). Subject to the BPS conditions, one obtains the following results,

$$
\begin{aligned}
& E_{\psi \theta \varphi}^{(I J)}=\frac{\sin \theta}{8 v^{2}}\left[-\mathrm{e}^{g} \sigma^{I} \sigma^{J} T_{01}+\sigma^{(I} W_{\psi}^{J)} T_{23}\right], \\
& E_{\psi r t}^{(I J)}=-\frac{1}{8 v^{2}}\left[\mathrm{e}^{g} \sigma^{I} \sigma^{J} T_{23}+\sigma^{(I} W_{\psi}^{J)} T_{01}\right], \\
& E_{r t \varphi}^{(I J)}=-\cos \theta \frac{T_{01} T_{23} \sigma^{I} \sigma^{J}}{32 v^{4}} \\
& E_{\theta t \varphi}^{(I J)}=r \sin \theta \frac{T_{01} T_{23} \sigma^{I} \sigma^{J}}{32 v^{4}}
\end{aligned}
$$


For $E_{\mu \nu \rho}^{\mathrm{W}}$, defined in (3.9), one derives the following equation upon using the BPS conditions,

$$
\begin{aligned}
E_{\mu \nu \rho}^{\mathrm{W}}= & -\frac{3}{16} \omega_{[\mu}^{a b}\left(\partial_{\nu} \omega_{\rho] a b}-\frac{2}{3} \omega_{\nu a c} \omega_{\rho]}^{c} b\right) \\
& -\frac{3}{4} \mathrm{i} e \varepsilon_{\mu \nu \rho \sigma \lambda}\left(T^{2} T^{\sigma \lambda}+6 T^{\sigma \kappa} T_{\kappa \tau} T^{\tau \lambda}\right) .
\end{aligned}
$$

This result leads to the following components,

$$
\begin{aligned}
E_{\psi \theta \varphi}^{\mathrm{W}} & =-\frac{3}{8} \sin \theta \mathrm{e}^{g} T_{01} . \\
E_{\psi r t}^{\mathrm{W}} & =\frac{3}{8} \mathrm{e}^{g} T_{23}, \\
E_{r t \varphi}^{\mathrm{W}} & =\cos \theta \frac{T_{01} T_{23}}{16 v^{2}}, \\
E_{\theta t \varphi}^{\mathrm{W}} & =-r \sin \theta \frac{T_{01} T_{23}}{16 v^{2}} .
\end{aligned}
$$

We note that the components listed in (4.40) and (4.42) are consistent with the fact that these three-forms are closed. Furthermore they are invariant under the scale transformations mentioned previously.

\section{The Lagrangian and the electric charges}

The construction of the relevant Lagrangian follows from the results presented in section 3 . Linear multiplets can be constructed from the products of two multiplets, which can then be coupled to a vector multiplet by using the superconformal density formula (3.10). The linear multiplet constructed from two vector multiplets will be written by means of a symmetric three-rank constant tensor $C_{I J K}$, which can be identified with the tensor that we introduced earlier in (4.1), although this is by no means essential. Below we will also use the notation $C(\sigma)=C_{I J K} \sigma^{I} \sigma^{J} \sigma^{K}$. The lowest component of the linear multiplet associated with the symmetrized product of two vector multiplets will thus be identified with $-C_{I J K} L^{i j(J K)}$, where we make use of (3.3). Higher components are defined accordingly. The vector multiplet that couples to the linear multiplet quadratic in the Weyl multiplet is characterized by constants $c_{I}$, so that its scalar field equals $c_{I} \sigma^{I}$. Finally we also include the Lagrangian for hypermultiplets.

After these definitions we introduce the expression for the bosonic terms in the Lagrangian, with convenient normalizations, decomposed according to,

$$
\mathcal{L}=\mathcal{L}_{\mathrm{vvv}}+\mathcal{L}_{\text {hyper }}+\mathcal{L}_{\mathrm{vww}}
$$

Here the Lagrangian cubic in vector multiplet fields equals,

$$
\begin{aligned}
8 \pi^{2} \mathcal{L}_{\mathrm{vvv}}= & 3 C_{I J K} \sigma^{I}\left[\frac{1}{2} \mathcal{D}_{\mu} \sigma^{J} \mathcal{D}^{\mu} \sigma^{K}+\frac{1}{4} F_{\mu \nu}{ }^{J} F^{\mu \nu K}-Y_{i j}{ }^{J} Y^{i j K}-3 \sigma^{J} F_{\mu \nu}{ }^{K} T^{\mu \nu}\right] \\
& -\frac{1}{8} \mathrm{i} C_{I J K} e^{-1} \varepsilon^{\mu \nu \rho \sigma \tau} W_{\mu}{ }^{I} F_{\nu \rho}{ }^{J} F_{\sigma \tau}{ }^{K}-C(\sigma)\left[\frac{1}{8} \mathcal{R}-4 D-\frac{39}{2} T^{2}\right],
\end{aligned}
$$


the Lagrangian for hypermultiplets (one of which plays the role of a compensating supermultiplet) reads,

$$
8 \pi^{2} \mathcal{L}_{\text {hyper }}=-\frac{1}{2} \Omega_{\alpha \beta} \varepsilon^{i j} \mathcal{D}_{\mu} A_{i}{ }^{\alpha} \mathcal{D}^{\mu} A_{j}{ }^{\beta}+\chi\left[\frac{3}{16} \mathcal{R}+2 D+\frac{3}{4} T^{2}\right],
$$

and the Lagrangian containing the higher-derivative couplings is given by,

$$
\begin{aligned}
8 \pi^{2} \mathcal{L}_{\mathrm{vww}}= & \frac{1}{4} c_{I} Y_{i j}{ }^{I} T^{a b} R_{a b k}{ }^{j}(V) \varepsilon^{k i} \\
& +c_{I} \sigma^{I}\left[\frac{1}{64} R_{a b}{ }^{c d}(M) R_{c d}{ }^{a b}(M)+\frac{1}{96} R_{a b j}{ }^{i}(V) R^{a b}{ }_{i}^{j}(V)\right] \\
& \left.-\frac{1}{128} \mathrm{i} e^{-1} \varepsilon^{\mu \nu \rho \sigma \tau} c_{I} W_{\mu}{ }^{I}\left[R_{\nu \rho}{ }^{a b}(M) R_{\sigma \tau a b}(M)+\frac{1}{3} R_{\nu \rho j}{ }^{i}(V) R_{\sigma \tau i}{ }^{j}(V)\right]\right] \\
& +\frac{3}{16} c_{I}\left(10 \sigma^{I} T_{a b}-F_{a b}{ }^{I}\right) R(M)_{c d}{ }^{a b} T^{c d} \\
& +c_{I} \sigma^{I}\left[3 T^{a b} \mathcal{D}^{c} \mathcal{D}_{a} T_{b c}-\frac{3}{2}\left(\mathcal{D}_{a} T_{b c}\right)^{2}+\frac{3}{2} \mathcal{D}_{c} T_{a b} \mathcal{D}^{a} T^{c b}+\mathcal{R}_{a b}\left(T^{a c} T^{b}{ }_{c}-\frac{1}{2} \eta^{a b} T^{2}\right)\right] \\
& +c_{I} \sigma^{I}\left[\frac{8}{3} D^{2}+8 T^{2} D-\frac{33}{8}\left(T^{2}\right)^{2}+\frac{81}{2}\left(T^{a c} T_{b c}\right)^{2}\right] \\
& -c_{I} F_{a b}{ }^{I}\left[T^{a b} D+\frac{3}{8} T^{a b} T^{2}-\frac{9}{2} T^{a c} T_{c d} T^{d b}\right] \\
& +\frac{3}{4} \mathrm{i} \varepsilon^{a b c d e}\left[c_{I} F_{a b}{ }^{I}\left(T_{c f} \mathcal{D}^{f} T_{d e}+\frac{3}{2} T_{c f} \mathcal{D}_{d} T_{e}^{f}\right)-3 c_{I} \sigma^{I} T_{a b} T_{c d} \mathcal{D}^{f} T_{f e}\right] .
\end{aligned}
$$

We remind the reader that $\mathcal{R}$ and $\mathcal{R}_{a b}$ refer to the Ricci scalar and tensor. The factor $8 \pi^{2}$, which equals four times the volume of the unit sphere $S^{3}$, has been included to avoid explicit factors of $\pi$ when defining electric charges. ${ }^{8}$ In the above result there are two terms which cannot be written in a manifestly gauge invariant form, related to the appearence of gravitational and SU(2) Chern-Simons terms. To avoid these Chern-Simons terms we have chosen to write their contribution in a form that is explicitly proportional to the gauge fields $W_{\mu}{ }^{I}$. This representation may lead to difficulty in case that the gauge fields are not globally defined, as we shall discuss in due course.

For future use we present the equation of motion for the auxiliary field $D$ that follows from the above results,

$$
\frac{16}{3} c_{I} \sigma^{I} D+c_{I}\left(8 \sigma^{I} T_{a b}-F_{a b}^{I}\right) T^{a b}+4 C(\sigma)+2 \chi=0 .
$$

On the horizon, this relation yields

$$
\chi=-2 C(\sigma)-2 c_{I} \sigma^{I} T^{2} .
$$

To appreciate the implications of the above results, let us first consider (5.2) for a single vector supermultiplet, so that $C(\sigma)=\sigma^{3}$. When suppressing the coupling to the fields $T_{a b}$,

\footnotetext{
${ }^{8}$ In four space-time dimensions one extracts a factor equal to two times the volume of the unit sphere $S^{2}$. In this way the Coulomb potential has the same normalization in four and in five dimensions, without factors of $\pi$.
} 
$D$ and to the Ricci scalar $R$, we are dealing with a Lagrangian based on a scalar field $\sigma$, a gauge field $W_{\mu}$ and an auxiliary field $Y^{i j}$. Upon re-introducing the fermion fields, this Lagrangian is invariant under rigid superconformal transformations. Note that the overall sign of the Lagrangian is irrelevant, as it can be absorbed into an overall sign of the vector supermultiplet fields. To identify the kinetic terms one may expand about some constant value of the field $\sigma$. The value of this constant is arbitrary and in fact it can be changed by a scale transformation that acts on all the fields and belongs to the rigid superconformal symmetry group. Note the presence of the Chern-Simons term, which implies that the corresponding action is only gauge invariant up to boundary terms.

When coupled to the fields of the Weyl multiplet, this Lagrangian is invariant under local superconformal transformations. However, it is inconsistent because the field $D$ acts as a Lagrange multiplier which requires $\sigma$ to vanish. To avoid this difficulty one must also introduce the superconformally invariant Lagrangian of a hypermultiplet. Introducing one hypermultiplet, the field equation for $D$ implies that $\chi=-2 \sigma^{3}$. In view of the local invariance under scale transformations $\sigma$ can be fixed to a constant. The phases contained in the hypermultiplet scalars can be fixed as well by making use of the local $\mathrm{SU}(2)$ transformations of the superconformal group, so that none of the scalar fields will correspond to physical degrees of freedom. Furthermore one can eliminate the auxiliary fields, $Y^{i j}$ and $T_{a b}$, by their (algebraic) field equations, which yields $Y^{i j}=0$ and $T_{a b}=$ $(4 \sigma)^{-1} F_{a b}$. Hence one is left with (for constant $\sigma$ ),

$$
8 \pi^{2} \mathcal{L}=-\frac{1}{2} \sigma^{3} \mathcal{R}-\frac{3}{8} \sigma F_{\mu \nu} F^{\mu \nu}-\frac{1}{8} \mathrm{i} e^{-1} \varepsilon^{\mu \nu \rho \sigma \tau} W_{\mu} F_{\nu \rho} F_{\sigma \tau}
$$

which, upon including the gravitino field (the other fermions are either auxiliary or can be set to zero by a gauge choice), is equal to the Lagrangian of pure five-dimensional supergravity. Observe that the vector gauge field is the only field of the vector multiplet and the hypermultiplet that describes physical degrees of freedom. The other ones are compensating fields (associated with scale transformations, R-symmetry and S-supersymmetry) or auxiliary fields. The field $\sigma$ is a constant and defines the Newton constant to be equal to $G_{\mathrm{N}}=\sigma^{-3}$, so that the Ricci scalar will appear in the Lagrangian with a multiplicative factor $\left(16 \pi^{2} G_{\mathrm{N}}\right)^{-1}$. This definition of Newton's constant is different from the more conventional one, where one adopts a prefactor $\left(16 \pi G_{\mathrm{N}}\right)^{-1}$, just as in four space-time dimensions. As a result of the convention of this paper, the Bekenstein-Hawking area law, leads to the area in Planck units, $A / G_{\mathrm{N}}$, with proportionality factor $(4 \pi)^{-1}$.

Let us briefly examine the relevant definition of the entropy defined in terms of the Noether potential [21, 23],

$$
\mathcal{S}_{\text {macro }}=2 \pi \int_{\Sigma_{\text {hor }}} \frac{\partial \mathcal{L}}{\partial \mathcal{R}_{\mu \nu \rho \sigma}} \varepsilon_{\mu \nu} \varepsilon_{\rho \sigma}
$$

where $\varepsilon_{\mu \nu}$ is the bi-normal tensor associated with the horizon, normalized such that $\varepsilon_{\mu \nu} \varepsilon^{\mu \nu}=-2$. For $\mathcal{L}=-\left(16 \pi^{2} G_{\mathrm{N}}\right)^{-1} \mathcal{R}$, this definition yields $\mathcal{S}_{\text {macro }}=\frac{1}{4}\left(A / \pi G_{\mathrm{N}}\right)$, which is the area law with the area described in units of $\pi G_{\mathrm{N}}$. This has a bearing on the various normalization factors for the Noether potential and the entropy discussed later on. 
In the next section we present a more detailed discussion of the entropy and the angular momentum. Before doing so we briefly discuss the electric charges, which follow from the relevant integral over the 3-cycle that encloses the black hole or the black ring,

$$
q_{I}=\frac{2}{8 \pi^{2}} \int \mathrm{d} \theta \mathrm{d} \varphi \mathrm{d} \psi\left[-3 C_{I J K} E_{\psi \theta \varphi}^{J K}+c_{I} E_{\psi \theta \varphi}^{\mathrm{W}}\right],
$$

where the relative factor 3 results from the fact that the Lagrangian (5.2) is cubic in the vector multiplets, whereas the Lagrangian (5.4) is only linear. An overall factor 2 has been included to be consistent with the usual definition of the charge in terms of the electric displacement field. Making use of the results (4.40) and (4.42), one obtains the following result,

$$
q_{I}=\frac{3}{2 v^{2}} C_{I J K}\left(\sigma^{J} \sigma^{K} \mathrm{e}^{g} T_{01}-\sigma^{J}\left[W_{\psi}^{K}\right] T_{23}\right)-\frac{3}{2} c_{I} \mathrm{e}^{g} T_{01},
$$

where we used the definition

$$
\left[W_{\psi}^{I}\right]=\frac{1}{16 \pi^{2}} \int \mathrm{d} \theta \mathrm{d} \varphi \mathrm{d} \psi \sin \theta W_{\psi}^{I} .
$$

which is gauge invariant under periodic gauge transformations. For spinning black holes, where the gauge fields are globally defined, (5.10) takes the form

$$
q_{I}=\frac{3 \mathrm{e}^{g}}{2 T_{01}}\left[C_{I J K} \sigma^{J} \sigma^{K}-c_{I} T_{01}^{2}\right] .
$$

Observe that the above results are scale invariant.

To derive the corresponding result for the black ring is more subtle in view of the fact that the gauge fields are not globally defined, as was discussed in subsection 4.3. This will be discussed in subsection 8 and the resulting expression for the charges will be given in (8.7).

The charges can also be determined by making use of the Noether potential associated with abelian gauge transformations. Consider, for instance, a Lagrangian in five space-time dimensions consisting of an invariant Lagrangian depending on the abelian field strength $F_{\mu \nu}$, its space-time derivatives $\nabla_{\rho} F_{\mu \nu}$, and matter fields denoted by $\psi$ and their derivatives $\nabla_{\mu} \psi$, plus an abelian Chern-Simons term,

$$
\mathcal{L}^{\text {total }}=\mathcal{L}^{\text {inv }}\left(F_{\mu \nu}, \nabla_{\rho} F_{\mu \nu}, \psi, \nabla_{\mu} \psi\right)+\varepsilon^{\mu \nu \rho \sigma \tau} A_{\mu} F_{\nu \rho} F_{\sigma \tau}
$$

For this Lagrangian, the Noether potential reads as follows,

$$
\mathcal{Q}_{\text {gauge }}^{\mu \nu}(\phi, \xi)=2 \mathcal{L}_{F}^{\mu \nu} \xi-2 \nabla_{\rho} \mathcal{L}_{F}^{\rho, \mu \nu} \xi+6 e^{-1} \varepsilon^{\mu \nu \rho \sigma \tau} \xi A_{\rho} F_{\sigma \tau},
$$

where $\phi$ generically denotes the various fields and $\xi$ is the infinitesimal local parameter associated with the gauge transformations. Here we use the notation,

$$
\delta \mathcal{L}^{\text {inv }}=\mathcal{L}_{F}^{\mu \nu} \delta F_{\mu \nu}+\mathcal{L}_{F}^{\rho, \mu \nu} \delta\left(\nabla_{\rho} F_{\mu \nu}\right)+\mathcal{L}_{\psi} \delta \psi+\mathcal{L}_{\psi}^{\mu} \delta\left(\nabla_{\mu} \psi\right)
$$

It is straightforward to verify that $\partial_{\nu} \mathcal{Q}^{\mu \nu}$ is equal to the field equation, up to terms proportional to $\partial_{\nu} \xi$. The electric charge can be written as

$$
q=\int_{\Sigma_{\mathrm{hor}}} \varepsilon_{\mu \nu} \mathcal{Q}_{\text {gauge }}^{\mu \nu}(\phi, \xi) .
$$


where $\varepsilon_{\mu \nu}$ is the binormal tensor associated with the horizon and the gauge parameter $\xi$ must be taken constant so that the underlying field configuration is invariant and the corresponding Noether current vanishes on-shell.

\section{Entropy and angular momentum for black holes and rings}

The evaluation of the entropy and the angular momentum proceeds from the expression for the Noether potential associated with space-time diffeomorphisms [21-23]. In the case at hand this is complicated in view of higher-derivative interactions, but it is especially subtle because of the presence of the Chern-Simons terms. At the end, one must evaluate the integral of the appropriate Noether potential over the horizon, and here one may encounter an extra subtlety when the gauge fields are not globally defined. This will be discussed further in section 8 .

In this section we start with a systematic discussion of the relevant Noether potential based on the Lagrangian specified in section 5. This Lagrangian contains two different Chern-Simons terms, one of the type $W \wedge F \wedge F$, which is cubic in the abelian gauge fields, and a mixed one of the type $W \wedge \operatorname{Tr}[\mathcal{R} \wedge \mathcal{R}]$, which is linear in the gauge fields and quadratic in the Riemann curvature. The derivation of the corresponding Noether potential is straightforward but subtle. We first evaluate this potential for a Lagrangian that depends on the Riemann tensor, on the field strengths of abelian gauge fields, and on an anti-symmetric tensor field $T_{\mu \nu}$ with at most first-order space-time derivatives $\nabla_{\mu} T_{\nu \rho}$. This Lagrangian does not contain the two Chern-Simons terms, which are considered separately. Its Noether potential associated with space-time diffeomorphisms decomposes into two different terms,

$$
\mathcal{Q}_{0}^{\mu \nu}=\hat{\mathcal{Q}}^{\mu \nu}\left(\xi^{\rho}\right)+\hat{\mathcal{Q}}_{\text {gauge }}^{\mu \nu}\left(-\xi^{\rho} W_{\rho}^{I}\right),
$$

corresponding to the following decomposition of the diffeomorphisms on the gauge field,

$$
\delta_{\xi} W_{\mu}^{I}=-\partial_{\mu} \xi^{\nu} W_{\nu}^{I}-\xi^{\nu} \partial_{\nu} W_{\mu}^{I}=\xi^{\nu} F_{\mu \nu}{ }^{I}+\partial_{\mu}\left(-\xi^{\nu} W_{\nu}{ }^{I}\right) .
$$

The first term, which does not contain the effect of the last term in (6.2), is given by

$$
\begin{aligned}
\hat{\mathcal{Q}}^{\mu \nu}\left(\xi^{\rho}\right)= & -2 \mathcal{L}_{\mathrm{R}}^{\mu \nu \rho \sigma} \nabla_{\rho} \xi_{\sigma}+4 \nabla_{\rho} \mathcal{L}_{\mathrm{R}}^{\mu \nu \rho \sigma} \xi_{\sigma} \\
& +\left[\mathcal{L}_{\mathrm{T}}^{\mu, \rho \sigma} T^{\nu}{ }_{\sigma}+\mathcal{L}_{\mathrm{T}}^{\rho, \mu \sigma} T^{\nu}{ }_{\sigma}+\mathcal{L}_{\mathrm{T}}^{\nu, \mu \sigma} T^{\rho}{ }_{\sigma}-(\mu \leftrightarrow \nu)\right] \xi_{\rho},
\end{aligned}
$$

where $\xi^{\rho}$ parametrizes the diffeomorphisms, and $\mathcal{L}^{\mu \nu \rho \sigma}$ and $\mathcal{L}^{\mu, \nu \rho}$ denote partial derivatives of the Lagrangian according to

$$
\delta \mathcal{L}=\mathcal{L}_{\mathrm{R}}^{\mu \nu \rho \sigma} \delta \mathcal{R}_{\mu \nu \rho \sigma}+\mathcal{L}_{\mathrm{T}}^{\mu, \nu \rho} \delta\left(\nabla_{\mu} T_{\nu \rho}\right) .
$$

These derivatives are subject to the BPS attractor equations. As a result they take the following form on the horizon,

$$
\begin{aligned}
8 \pi^{2} \mathcal{L}_{\mathrm{R}}^{\mu \nu \rho \sigma} & =\left(-\frac{1}{2} C(\sigma)-\frac{3}{4} c_{I} \sigma^{I} T^{2}\right) g^{\mu[\rho} g^{\sigma] \nu}+\frac{1}{2} c_{I} \sigma^{I}\left(T^{\mu \nu} T^{\rho \sigma}-T^{\mu[\rho} T^{\sigma] \nu}\right) \\
8 \pi^{2} \mathcal{L}_{\mathrm{T}}^{\rho, \mu \nu} & =-3 c_{I} \sigma^{I}\left(3 \mathcal{D}^{[\mu} T^{\nu] \rho}+\mathcal{D}^{\rho} T^{\mu \nu}+\frac{5}{4} \mathrm{i} \varepsilon^{\mu \nu \sigma \lambda \tau} T_{\sigma \lambda} T_{\tau}{ }^{\rho}\right) \\
& =-\frac{9}{4} \mathrm{i} c_{I} \sigma^{I} \varepsilon^{\mu \nu \sigma \lambda \tau} T_{\sigma \lambda} T_{\tau}{ }^{\rho}
\end{aligned}
$$


Obviously we also need the derivative $\nabla_{\rho} \mathcal{L}_{\mathrm{R}}^{\mu \nu \rho \sigma}$, which follows form (6.5) by means of the attractor equations. The result reads as follows,

$$
8 \pi^{2} \nabla_{\rho} \mathcal{L}_{\mathrm{R}}^{\mu \nu \rho \sigma}=\frac{5}{8} \mathrm{i} c_{I} \sigma^{I}\left(T^{\mu \nu} \varepsilon^{\sigma \rho \lambda \kappa \tau}-T^{\sigma[\mu} \varepsilon^{\nu] \rho \lambda \kappa \tau}\right) T_{\rho \lambda} T_{\kappa \tau}
$$

It remains to consider the second term in (6.1), $\hat{\mathcal{Q}}_{\text {gauge }}^{\mu \nu}\left(-\xi^{\rho} W_{\rho}^{I}\right)$, which denotes the Noether potential associated with the abelian gauge transformations with field-dependent gauge parameters $\xi^{I}=-\xi^{\rho} W_{\rho}{ }^{I}$. This potential was already presented in (5.14), where the last term corresponding to the $W \wedge F \wedge F$ Chern-Simons term has been suppressed. We thus need the expression for $\mathcal{L}_{\mathrm{F} I}^{\mu \nu}$ (c.f. (5.15)),

$$
\begin{aligned}
8 \pi^{2} \mathcal{L}_{\mathrm{F} I}^{\mu \nu}= & \frac{3}{2} C_{I J K} \sigma^{J} F^{\mu \nu K}-9 C_{I J K} \sigma^{J} \sigma^{K} T^{\mu \nu} \\
& -\frac{3}{16} c_{I} R_{a b}{ }^{\mu \nu}(M) T^{a b}+\frac{3}{4} \mathrm{i} c_{I} \varepsilon^{\mu \nu \rho \sigma \tau} T_{\rho \lambda}\left(\mathcal{D}^{\lambda} T_{\sigma \tau}+\frac{3}{2} \mathcal{D}_{\sigma} T_{\tau}{ }^{\lambda}\right) \\
& -c_{I}\left[T^{\mu \nu}\left(D+\frac{3}{8} T^{2}\right)-\frac{9}{2} T^{\mu \rho} T_{\rho \sigma} T^{\sigma \nu}\right] \\
= & -3 C_{I J K} \sigma^{J} \sigma^{K} T^{\mu \nu}+\frac{3}{4} c_{I}\left(T^{\mu \nu} T^{2}+6 T^{\mu \rho} T_{\rho \sigma} T^{\sigma \nu}\right)
\end{aligned}
$$

where the second equation represents the value taken at the horizon.

By combining the above contributions we obtain an explicit expression for (6.1). In practice we need the contraction of the Noether potential with the bi-normal tensor (4.28) associated with the horizon. Therefore we evaluate the following expression for (6.1),

$$
\begin{aligned}
8 \pi^{2} \varepsilon_{\mu \nu} \mathcal{Q}_{0}^{\mu \nu}= & -2 \varepsilon_{01} C(\sigma) \nabla_{[0} \xi_{1]} \\
& -2 \varepsilon_{01} c_{I} \sigma^{I}\left[3 T_{23}{ }^{2} \nabla_{[0} \xi_{1]}-2 T_{01} T_{23} \nabla_{[2} \xi_{3]}+11 T_{01}{ }^{2} T_{23} \xi_{5}\right] \\
& +2 \varepsilon_{01} \xi^{\rho} W_{\rho}^{I} T_{01}\left[-6 C_{I J K} \sigma^{J} \sigma^{K}+3 c_{I}\left(T_{23}{ }^{2}+2 T_{01}{ }^{2}\right)\right]
\end{aligned}
$$

Subsequently, we turn to the Chern-Simons terms contained in (5.2) and (5.4),

$$
8 \pi^{2} \mathcal{L}_{\mathrm{CS}}=-\frac{1}{8} \mathrm{i} e^{-1} \varepsilon^{\mu \nu \rho \sigma \tau}\left[C_{I J K} W_{\mu}{ }^{I} F_{\nu \rho}{ }^{J} F_{\sigma \tau}{ }^{K}+\frac{1}{16} c_{I} W_{\mu}{ }^{I} \mathcal{R}_{\nu \rho}{ }^{a b} \mathcal{R}_{\sigma \tau a b}\right],
$$

which contribute to the Noether potential as follows,

$$
\begin{aligned}
8 \pi^{2} \mathcal{Q}_{\mathrm{CS}}^{\mu \nu}= & \frac{1}{2} \mathrm{i} e^{-1} \varepsilon^{\mu \nu \rho \sigma \tau} C_{I J K} \xi^{\lambda} W_{\lambda}^{I} W_{\rho}^{J} F_{\sigma \tau}{ }^{K} \\
& +\frac{1}{32} \mathrm{i} e^{-1} \varepsilon^{\mu \nu \rho \sigma \tau} c_{I} W_{\rho}{ }^{I} \mathcal{R}_{\sigma \tau}{ }^{\kappa \lambda} \nabla_{\kappa} \xi_{\lambda} \\
& -\frac{1}{32} \mathrm{i} e^{-1} \varepsilon^{\rho \sigma \tau \lambda[\mu} c_{I} F_{\rho \sigma}{ }^{I} \mathcal{R}_{\tau \lambda}{ }^{\nu] \kappa} \xi_{\kappa} \\
& +\frac{1}{64} \mathrm{i} e^{-1} \varepsilon^{\rho \sigma \tau \lambda \kappa} c_{I} F_{\rho \sigma}{ }^{I} \mathcal{R}_{\tau \lambda}{ }^{\mu \nu} \xi_{\kappa} .
\end{aligned}
$$

We note that the first term is similar to what one expects from the expression for the Noether potential associated with gauge transformations. However, it carries a different 
pre-factor than in (5.14), a feature that is well known (see e.g. [60]). Evaluating this expression at the horizon, using (4.7) and (4.34), yields,

$$
\begin{aligned}
8 \pi^{2} \varepsilon_{\mu \nu} \mathcal{Q}_{\mathrm{CS}}^{\mu \nu}= & 8 \varepsilon_{01} T_{23} C_{I J K} \sigma^{I} W_{5}{ }^{J} W_{\lambda}{ }^{K} \xi^{\lambda} \\
& -\varepsilon_{01} c_{I} W_{5}{ }^{I}\left[-2 T_{01} T_{23} \nabla_{[0} \xi_{1]}+\left(T_{01}{ }^{2}+4 T_{23}{ }^{2}\right) \nabla_{[2} \xi_{3]}\right] \\
& -\varepsilon_{01} T_{01}{ }^{2} c_{I}\left[W_{3}{ }^{I} \nabla_{[5} \xi_{2]}-W_{2}{ }^{I} \nabla_{[5} \xi_{3]}\right] \\
& +2 \varepsilon_{01} c_{I} \sigma^{I} T_{23}\left[6 T_{01}{ }^{2}-T_{23}{ }^{2}\right] \xi_{5} .
\end{aligned}
$$

Note that the above results depend explicitly on the gauge fields $W_{\mu}{ }^{I}$. For black holes, where the gauge fields are globally defined, this is not an issue. However, for black rings the situation is different and extra care is required. As we discuss in subsection 6.1 we will employ an alternative treatment of the mixed Chern-Simons term which will lead to expressions that differ from (6.9)-(6.11).

By integrating the Noether potential over the horizon one obtains the entropy and the angular momentum from the Noether potential associated with the relevant Killing vector and contracted with the bi-normal tensor (4.28). For the entropy the relevant Killing vector is the timelike one, $\xi^{\mu} \partial_{\mu}=\partial / \partial t$, and in the integrand one drops all terms except the ones proportional to $\nabla_{[0} \xi_{1]}=\varepsilon_{01}$,

$$
\mathcal{S}_{\text {macro }}=-\left.\pi \int_{\Sigma_{\text {hor }}} \varepsilon_{\mu \nu} \mathcal{Q}^{\mu \nu}(\xi)\right|_{\nabla_{[\mu} \xi_{\nu]}=\varepsilon_{\mu \nu} ; \xi^{\mu}=0},
$$

For the angular momentum the Killing vector is associated with the corresponding periodic isometry of the space-time, and one has

$$
J(\xi)=\int_{\Sigma_{\text {hor }}} \varepsilon_{\mu \nu} \mathcal{Q}^{\mu \nu}(\xi) .
$$

\subsection{An alternative form of the mixed Chern-Simons term}

In the above derivation of the Noether potential, we were able to handle the mixed ChernSimons term by writing it in the form $W \wedge \operatorname{Tr}[\mathcal{R} \wedge \mathcal{R}]$, so that the Lagrangian is manifestly diffeomorphism covariant at the expense of introducing explicit gauge fields $W_{\mu}{ }^{I}$. This is acceptable in cases where the gauge fields are globally defined, such as for spinning black holes. In the case of a black ring, however, the presence of magnetic charges implies that the gauge fields are only defined in patches, making the use of the above formulae questionable.

Therefore we consider an alternative derivation, based on a modification of the Lagrangian (5.4) proportional to $\varepsilon^{\mu \nu \rho \sigma \tau} W_{\mu}{ }^{I} \mathcal{R}_{\nu \rho}{ }^{a b} \mathcal{R}_{\sigma \tau a b}$, by adding a suitable total derivative. In this way the gauge field is converted to its field strength (which is globally defined), and the square of the curvature tensor $\mathcal{R}$ is converted to a corresponding Chern-Simons term. The alternative form of the mixed Chern-Simons term is thus, ${ }^{9}$

$$
8 \pi^{2} \mathcal{L}_{\mathrm{CS}}=-\frac{1}{64} \mathrm{i} e^{-1} \varepsilon^{\mu \nu \rho \sigma \tau} c_{I} F_{\mu \nu}{ }^{I} \omega_{\rho}^{a b}\left(\partial_{\sigma} \omega_{\tau a b}-\frac{2}{3} \omega_{\sigma a c} \omega_{\tau}^{c} b\right)
$$

\footnotetext{
${ }^{9}$ Note that in this subsection we suppress the $W \wedge F \wedge F$ Chern-Simons term of (6.9), which is not affected by the conversion and whose effect has already been evaluated.
} 
From the point of view of general coordinate invariance, this change does not seem crucial, as the Lagrangian (6.14) still transforms as a scalar. On the other hand, the spin-connection field $\omega_{\mu}^{a b}$ is a composite vector field associated with local Lorentz transformations. As a result of the explicit spin-connection, this form of the Lagrangian is no longer invariant under local Lorentz transformations, but transforms into a boundary term.

In this formulation diffeomorphism invariance of the relevant field configurations will be defined up to a local Lorentz transformation. Therefore Lorentz transformations have to be taken into account in the relevant Noether potential. In the previous form of the mixed Chern-Simons term given in (6.9), the local Lorentz transformations were avoided because that expression can be interpreted directly in the metric formulation without the need for including vielbein fields. In principle, the invariance of the field configuration could require additional components associated with gauge transformations other than the Lorentz transformations, but the gauge fields turn out to be invariant under the relevant diffeomorphisms without the need for including compensating gauge transformations. Therefore it suffices to consider only diffeomorphisms and local Lorentz transformations in the following.

Under the combined variation of a diffeomorphism and a local Lorentz transformation with parameters $\xi^{\mu}$ and $\varepsilon^{a b}$, the Lagrangian (6.14) corresponding to the mixed ChernSimons term transforms as

$$
8 \pi^{2} \delta\left(\sqrt{g} \mathcal{L}_{\mathrm{CS}}\right)=-\partial_{\mu}\left(\xi^{\mu} \sqrt{g} \mathcal{L}_{\mathrm{CS}}-\frac{1}{64} \mathrm{i} \varepsilon^{\mu \nu \rho \sigma \tau} c_{I} F_{\nu \rho}{ }^{I} \partial_{\sigma} \varepsilon_{a b} \omega_{\tau}^{a b}\right)
$$

The corresponding Noether potential depending on both $\xi^{\mu}$ and $\varepsilon_{a b}$, is then equal to

$$
\begin{aligned}
8 \pi^{2} \mathcal{Q}_{\mathrm{CS}}^{\mu \nu}= & -\frac{1}{32} \mathrm{i} e^{-1} \varepsilon^{\mu \nu \rho \sigma \tau} c_{I} F_{\rho \sigma}{ }^{I} \omega_{\tau}^{a b}\left[\varepsilon_{a b}-\frac{1}{2} \xi^{\kappa} \omega_{\kappa a b}\right] \\
& +\frac{1}{32} \mathrm{i} e^{-1} \varepsilon^{\mu \nu \rho \sigma \tau} c_{I} \xi^{\lambda} W_{\lambda}^{I} \omega_{\rho}^{a b}\left(\partial_{\sigma} \omega_{\tau a b}-\frac{2}{3} \omega_{\sigma a c} \omega_{\tau}^{c} b\right) \\
& -\frac{1}{32} \mathrm{i} e^{-1} \varepsilon^{\rho \sigma \tau \lambda[\mu} c_{I} F_{\rho \sigma}{ }^{I} \mathcal{R}_{\tau \lambda}{ }^{\nu] \kappa} \xi_{\kappa} \\
& +\frac{1}{64} \mathrm{i} e^{-1} \varepsilon^{\rho \sigma \tau \lambda \kappa} c_{I} F_{\rho \sigma}{ }^{I} \mathcal{R}_{\tau \lambda}{ }^{\mu \nu} \xi_{\kappa} .
\end{aligned}
$$

We note that the last two covariant terms proportional to $F \wedge R$ are identical to the corresponding terms given in (6.10). This expression should be evaluated for backgrounds that are invariant, which implies that the transformation parameter $\varepsilon^{a b}$ should be chosen such that the vielbein is invariant under the diffeomorphisms. This implies that the diffeomorphism is again generated by a Killing vector $\xi^{\mu}$, and

$$
\varepsilon^{a b}=-\nabla^{[a} \xi^{b]}+\xi^{\lambda} \omega_{\lambda}^{a b} .
$$

This result for $\varepsilon^{a b}$ should be substituted into the expression (6.16) for the Noether potential. The resulting expression is then expected to match the previous result (6.10) (without the contribution of the $W \wedge F \wedge F$ Chern-Simons term which has not been included above), when both the gauge fields and the spin connection field can be globally defined. This 
is not the case for the black hole and black ring solutions, so that only one of the two expressions will be applicable in either case. It should be of interest to compare the two formulae in more detail by making explicit use of coordinate patches.

We should, however, briefly comment on the ambiguity in (6.16) related to the fact that the extraction of the derivative $\partial_{\mu}$ in (6.15) is not well motivated for the second term, as we could have also left the derivative on the spin connection field $\omega_{\tau}{ }^{a b}$ and extracted the derivative from the transformation parameter $\varepsilon^{a b}$. The choice made above can be justified along the lines of [24], which is consistent with the original description of Wald [21, 23]. One considers the variation of the corresponding Noether current under a continuous change in the space of solutions of the field equations, in order to derive the first law of black hole mechanics. For the current relevant in this section, this variation equals,

$$
\begin{aligned}
8 \pi^{2} \delta J^{\mu}(\phi, \xi, \varepsilon)= & \partial_{\nu}\left[\xi^{\mu} \theta^{\nu}(\phi, \delta \phi)-\xi^{\nu} \theta^{\mu}(\phi, \delta \phi)\right]+\omega\left(\phi ; \delta_{\xi} \phi, \delta \phi\right) \\
& -\frac{1}{8} \mathrm{i} \partial_{\nu}\left[\varepsilon^{\mu \nu \rho \sigma \tau} c_{I} \delta W_{\rho}^{I} \partial_{\sigma} \varepsilon^{a b} \omega_{\tau a b}\right]
\end{aligned}
$$

where $\xi^{\mu}$ parametrizes a diffeomorphism and $\varepsilon^{a b}$ a Lorentz transformation, while the variation $\delta_{\xi}$ is defined as the combined effect of both transformations. The variations $\delta \phi$ and $\delta W_{\mu}^{I}$ connect two nearby solutions. At this point the diffeomorphism and the Lorentz transformation are arbitrary and do not have to constitute an invariance of the field configuration.

The first two terms on the right-hand side are generic. The first one, proportional to the divergence of $\xi^{[\mu} \theta^{\nu]}$, should be written as the variation of another term, which can then be included into the Noether potential. This modification will not change the entropy because it does not involve derivatives of $\xi^{\mu}$, and furthermore it does not contribute to the variations of the angular momenta at spatial infinity [23]. Actually, the form of this term ensures that the angular momenta can be determined from the Noether potential and remain constant as a function of the distance from the horizon [60].

The second term is equal to $\omega\left(\phi, \delta_{1} \phi ; \delta_{2} \phi\right)=\delta_{2} \theta\left(\phi, \delta_{1} \phi\right)-\delta_{1} \theta\left(\phi, \delta_{2} \phi\right)$, where $\delta_{1} \phi$ and $\delta_{2} \phi$ denote independent field variations. When $\xi^{\mu}$ is the time evolution field, then the integral of this quantity over a Cauchy surface will be equal to the corresponding Hamiltonian in the covariant phase-space approach. The variation of the ADM mass follows from this Hamiltonian, and the modification to the Noether potential related to $\xi^{[\mu} \theta^{\nu]}$ will thus contribute to it. It is also relevant that $\omega\left(\phi ; \delta_{\xi} \phi, \delta \phi\right)$ will vanish for symmetric field configuration, because $\delta_{\xi} \phi=0$ in that case.

The hope is that the third term in (6.18) will behave in the same way. This term will also lead to modifications of the Noether potential, and since it depends on $\xi^{\mu}$ as well as on its derivatives, these modifications may affect the entropy. However, it is easy to see that this will not be the case, because the relevant $\varepsilon^{a b}$ at the horizon is precisely the bi-normal tensor (4.28), whose derivatives vanish. Therefore the third term in (6.18) will not lead to extra terms in the entropy. For the angular momenta, the situation is similar but more subtle. In that case the combination $\partial_{\sigma} \varepsilon^{a b} \omega_{\tau a b}$ vanishes at the horizon, except for $\partial_{\theta} \varepsilon^{a b} \omega_{\varphi a b} \propto \sin \theta \cos \theta$. Therefore this term vanishes upon integration over the horizon for all $\delta W_{\rho}^{I}$ that are allowed by the attractor equations. Hence the angular momenta at 
the horizon are not modified and can be determined from the Noether potential obtained earlier. An obvious question is, whether the angular momentum whose variation appears in the first law, and which is measured at spatial infinity, will coincide with the angular momenta determined at the horizon. The answer to this question is not known, but the results that we will present in section 8 indicate that the answer is affirmative. Obviously a full derivation of the first law for the ring geometry is subtle in the presence of higherderivative couplings. Without the latter, the derivation of the first law has already been pursued in [61] in connection with the presence of the dipole charges.

\section{Spinning BPS black holes}

In this section we apply the material derived in the preceding sections to the case of spinning black holes. Subsequently we discuss various implications of our results and compare them to results that have been obtained elsewhere.

We assume arbitrary non-zero values of $p^{0}$. Using (4.27), we integrate the Noether potential derived in (6.8) and (6.11) over the horizon. In this way we obtain the following expression for the entropy,

$$
\mathcal{S}_{\text {macro }}^{\mathrm{BH}}=\frac{\pi \mathrm{e}^{g}}{4 v^{2}}\left[C(\sigma)+4 c_{I} \sigma^{I} T_{23}^{2}\right] .
$$

The moduli are expressed in terms of the angular momentum $J_{\psi}$ and the charges $q_{I}$ and $p^{0}$ by the attractor equations. The black holes have only one component of angular momentum, associated with the Killing vector $\xi^{\mu} \partial_{\mu}=\partial / \partial \psi$. Here we refrain from introducing any additional normalization factor. This leads to $\xi_{5}=\mathrm{e}^{g}$ and

$$
\nabla_{[0} \xi_{1]}=2 T_{23} \mathrm{e}^{g}, \quad \nabla_{[2} \xi_{3]}=-2 T_{01} \mathrm{e}^{g} .
$$

Substituting these results into (6.8) and (6.11), and setting $\varepsilon_{01}=1$, yields the following expression for $J_{\psi}$,

$$
J_{\psi}=\frac{T_{23} \mathrm{e}^{2 g}}{T_{01}^{2}}\left[C_{I J K} \sigma^{I} \sigma^{J} \sigma^{K}-4 c_{I} \sigma^{I} T_{01}^{2}\right] .
$$

Note that there is no other non-vanishing component of angular momentum in this case. The charges follow from (5.10) and (4.32),

$$
q_{I}=\frac{3 \mathrm{e}^{g}}{2 T_{01}}\left[C_{I J K} \sigma^{J} \sigma^{K}-c_{I} T_{01}^{2}\right], \quad p^{0}=\frac{\mathrm{e}^{-g}}{4 v^{2}} T_{01} .
$$

It is convenient to express these results in terms of scale invariant variables defined by

$$
\phi^{I}=\frac{\sigma^{I}}{4 T_{01}}, \quad \phi^{0}=\frac{e^{-g} T_{23}}{4 v^{2}}=\frac{p^{0} T_{23}}{T_{01}} .
$$

In terms of these variables (7.1) reads

$$
\mathcal{S}_{\text {macro }}^{\mathrm{BH}}=\frac{4 \pi p^{0}}{\left(\phi^{0^{2}}+p^{0^{2}}\right)^{2}}\left[p^{0^{2}} C_{I J K} \phi^{I} \phi^{J} \phi^{K}+\frac{1}{4} c_{I} \phi^{I} \phi^{0^{2}}\right],
$$


whereas the attractor equations for the electric charges $q_{I}$ and the angular momentum $J_{\psi}$ take the form,

$$
\begin{aligned}
q_{I} & =\frac{6 p^{0}}{\phi^{0^{2}}+p^{0^{2}}}\left[C_{I J K} \phi^{J} \phi^{K}-\frac{1}{16} c_{I}\right], \\
J_{\psi} & =\frac{4 \phi^{0} p^{0}}{\left(\phi^{0^{2}}+p^{0^{2}}\right)^{2}}\left[C_{I J K} \phi^{I} \phi^{J} \phi^{K}-\frac{1}{4} c_{I} \phi^{I}\right] .
\end{aligned}
$$

This result shows that $\phi^{0}$ is proportional to the angular momentum, as is also obvious from (7.5). To understand the limit in which the charges become uniformly large, we consider uniform rescalings of the charges $q_{I}$ and $p^{0}$ as well as of the moduli $\phi^{I}$ and $\phi^{0}$. Obviously, the terms proportional to $C_{I J K}$ in the attractor equations are consistent with this scaling, whereas the terms proportional to $c_{I}$ are suppressed inversely proportional to the square of the charges and thus represent subleading contributions. The leading term of the entropy then scales as the square of the charges, while the correction terms proportional to $c_{I}$, which originate from the higher-order derivative couplings, represent the subleading contributions in the limit where all charges become large.

The above results can be compared to the corresponding results in four space-time dimensions. The relevant holomorphic and homogeneous function in which the supergravity action is encoded takes the form,

$$
F(Y, \Upsilon)=\frac{D_{I J K} Y^{I} Y^{J} Y^{K}+d_{I} Y^{I} \Upsilon}{Y^{0}}
$$

Here $Y^{I}$ and $Y^{0}$ are holomorphic variables related to the scalar fields of the four-dimensional vector multiplets and $\Upsilon$ is the lowest component of the square of the Weyl multiplet, all subject to some uniform rescaling. The quantities $D_{I J K}$ and $d_{I}$ should be identified with $C_{I J K}$ and $c_{I}$, up to suitable proportionality factors. In terms of these variables the attractor equations read $[4,54,62]$,

$$
Y^{A}-\bar{Y}^{A}=\mathrm{i} p^{A}, \quad F_{A}-\bar{F}_{A}=\mathrm{i} q_{A},
$$

where the index $A$ denotes $A=0$ or $A=I$ and where the magnetic and electric charges are denoted by $p^{A}$ and $q_{A}$, respectively. Furthermore the BPS condition implies $\Upsilon=-64$.

It is well-known that the theory based on (7.8) is invariant under the following symmetry transformations, which take the form of electric/magnetic dualities (see, e.g. [63]),

$$
\begin{aligned}
& Y^{0} \rightarrow Y^{0} \\
& Y^{I} \rightarrow Y^{I}+k^{I} Y^{0} \\
& F_{0} \rightarrow F_{0}-k^{I} F_{I}-3 D_{I J K} k^{J} k^{K} Y^{I}-D_{I J K} k^{I} k^{J} k^{K} Y^{0}, \\
& F_{I} \rightarrow F_{I}+6 D_{I J K} k^{J} Y^{K}+3 D_{I J K} k^{J} k^{K} Y^{0}
\end{aligned}
$$

where the parameters $k^{I}$ are real. In principle there could be other dualities as well, depending on the specific form of the coefficients $C_{I J K}$ and $c_{I}$. The electric and magnetic 
charges will exhibit similar transformations,

$$
\begin{aligned}
& p^{0} \rightarrow p^{0}, \\
& p^{I} \rightarrow p^{I}+k^{I} p^{0}, \\
& q_{0} \rightarrow q_{0}-k^{I} q_{I}-3 D_{I J K} k^{J} k^{K} p^{I}-D_{I J K} k^{I} k^{J} k^{K} p^{0}, \\
& q_{I} \rightarrow q_{I}+6 D_{I J K} k^{J} p^{K}+3 D_{I J K} k^{J} k^{K} p^{0} .
\end{aligned}
$$

Parametrizing the $Y^{A}$ by $Y^{A}=\frac{1}{2}\left(\phi^{A}+\mathrm{i} p^{A}\right)$, so that the magnetic attractor equations of (7.9) are satisfied, we obtain the following expressions for the entropy and the remaining attractor equations for $p^{I}=0$ and $p^{0} \neq 0$,

$$
\mathcal{S}_{4 \mathrm{D}}^{\mathrm{BH}}=\frac{2 \pi p^{0}}{\left(\phi^{0^{2}}+p^{0^{2}}\right)^{2}}\left[p^{0^{2}} D_{I J K} \phi^{I} \phi^{J} \phi^{K}+256 d_{I} \phi^{I} \phi^{0^{2}}\right],
$$

with

$$
\begin{aligned}
q_{I}^{4 \mathrm{D}} & =-\frac{3 p^{0}}{\phi^{0^{2}}+p^{0^{2}}}\left[D_{I J K} \phi^{J} \phi^{K}-\frac{256}{3} d_{I}\right], \\
q_{0}{ }^{4 \mathrm{D}} & =\frac{2 \phi^{0} p^{0}}{\left(\phi^{0^{2}}+p^{0^{2}}\right)^{2}}\left[D_{I J K} \phi^{I} \phi^{J} \phi^{K}-256 d_{I} \phi^{I}\right] .
\end{aligned}
$$

The symmetry discussed in (7.10) and (7.11) is not manifest for the above result, in view of the fact that we have fixed the $p^{I}$ to zero. However, it can be used to find the corresponding expressions for non-zero charges $p^{I}$.

Without giving a precise calibration between four- and five-dimensional quantities (which is subtle in the presence of higher-derivative couplings) it is clear that the fourand five-dimensional expressions are not compatible upon absorbing suitable normalization factors in the quantities involved. The difference seems to reside exclusively in the attractor equations for the electric charges $q_{I}$, in the terms proportional to $c_{I}$ induced by the higherderivative couplings. The expressions for entropy and angular momentum agree assuming that the charge $q_{0}{ }^{4 \mathrm{D}}$ is identified with $J_{\psi}$. These results are different from those obtained in [16], especially in the case of non-zero angular momentum. For details, we refer to the discussion at the end of this subsection.

To investigate some of the consequences of this discrepancy, we again consider the attractor equations (7.7), where we rescale the coefficients $c_{I}$ in the attractor equations for $q_{I}$ by $c_{I} \rightarrow \alpha c_{I}$ to account for the two different expressions. Hence we set the parameter $\alpha=$ 1 or $\frac{4}{3}$, depending on whether we consider $D=5$ or 4 space-time dimensions, respectively.

Subsequently we solve the attractor equations for $\phi^{I}$ and $\phi^{0}$ to first order in $c_{I}$, keeping the charges constant. To do this we first determine the solution for the case that $c_{I}=0$,

$$
\begin{aligned}
& \hat{\phi}^{I} \equiv \frac{\phi^{I}}{\sqrt{\phi^{0^{2}}+p^{0^{2}}}} \approx \frac{\hat{q}^{I}}{\sqrt{p^{0}}}+\mathcal{O}\left(c_{I}\right), \\
& \phi^{0} \approx \frac{J_{\psi} p^{0^{2}}}{2 \sqrt{p^{0} Q^{3}-\frac{1}{4}\left(p^{0} J_{\psi}\right)^{2}}}+\mathcal{O}\left(c_{I}\right),
\end{aligned}
$$


where the $\hat{q}^{I}$ are defined by the requirement that they satisfy the attractor equations in the limit of vanishing $c_{I}$. Therefore we have,

$$
\begin{aligned}
q_{I} & =6 C_{I J K} \hat{q}^{J} \hat{q}^{K}, \\
Q^{3 / 2} & =2 C_{I J} \hat{q}^{J} \hat{q}^{K}, \\
C_{I J} & =C_{I J K} \hat{q}^{K} .
\end{aligned}
$$

To first order in $c_{I}$ this result changes into,

$$
\begin{aligned}
\hat{\phi}^{I} & \approx \frac{1}{\sqrt{p^{0}}}\left\{\hat{q}^{I}+\frac{\alpha\left(p^{0} Q^{3}-\frac{1}{4}\left(p^{0} J_{\psi}\right)^{2}\right)}{32 p^{0^{2}} Q^{3}} C^{I J} c_{J}\right\}+\mathcal{O}\left(c_{I}^{2}\right), \\
\phi^{0} & \approx \frac{J_{\psi} p^{0^{2}}}{2 \sqrt{p^{0} Q^{3}-\frac{1}{4}\left(p^{0} J_{\psi}\right)^{2}}}\left\{1-\frac{(3 \alpha-8) c_{I} \hat{q}^{I}}{16 p^{0} Q^{3 / 2}}\right\}+\mathcal{O}\left(c_{I}^{2}\right),
\end{aligned}
$$

where the matrix $C^{I J}$ denotes the inverse of $C_{I J}$. Substituting these expressions into the entropy formula (7.6), one obtains,

$$
\mathcal{S}_{\text {macro }}^{\mathrm{BH}} \approx 2 \pi \sqrt{p^{0} Q^{3}-\frac{1}{4}\left(p^{0} J_{\psi}\right)^{2}}\left(1+\frac{3 \alpha}{16} \frac{c_{I} \hat{q}^{I}}{p^{0} Q^{3 / 2}}\right)+\mathcal{O}\left(c_{I}^{2}\right) .
$$

We note that the terms proportional to $c_{I}$ are indeed subleading in the limit of large charges.

The expression (7.17) can be confronted with results from the literature. For the nonrotating case, where a direct comparison with microscopic counting is possible, the above result with $\alpha=1$ agrees with the results reported in [26, 27]. For the rotating black hole, no analytic microscopic results are available, but our results can be compared to the supergravity results of $[16,17]$. Here there is a clear discrepancy originating from the different form of the attractor equations (7.3) for the electric charges and the angular momentum, which reflects itself in a different dependence on $J$ in (7.17). The expression (7.17) can also be compared to the results of [36], where the only higher-derivative coupling included into the action was the Euler density. For zero angular momentum one recovers the same relative factor for the subleading correction between the four- and five-dimensional entropies represented by the parameter $\alpha$ in (7.17). For finite angular momentum the subleading corrections determined by $[16,17]$ and $[36]$ are mutually different and both fail to reproduce the expression (7.17). As already mentioned in footnote 2 there exist microscopic results for theories with 16 supercharges [30, 31], which could possibly be connected to the results of this paper in certain asymptotic limits.

Irrespective of the discrepancies in the rotating case, our five-dimensional results, as well as those in $[16,17,26,27]$ disagree with a naive uplift of the four-dimensional result. Restricting ourselves to the static case, it appears that the $\frac{4}{3}$ discrepancy in the attractor equation for the charges is ubiquitous. A possible origin of these discrepancies follows from the observation that the actions used in four and in five dimensions are not directly related by dimensional reduction. Upon reduction the five-dimensional Weyl multiplet decomposes into the four-dimensional Weyl multiplet and a four-dimensional vector multiplet, as is obvious from table 3. Therefore we expect that the resulting four-dimensional action 
will also contain special higher-derivative couplings involving vector multiplets that have, so far, not been considered in this context. Apparently these terms do not change the expression for the Wald entropy expressed in the moduli. The latter is in accord with a result of [64], where the construction of higher-derivative Lagrangians for tensor multiplets leads to additional couplings to the Riemann tensor which all vanish in the BPS limit, so that they will not contribute to the Wald entropy. Assuming that this phenomenon holds in the general case, then the higher-order couplings of the vector multiplets may still generate new contributions to the electric charges or to the angular momentum. At present these couplings are not completely known so it is difficult to check whether or not this is the correct explanation for the discrepancy. Observe that this phenomenon will not arise for models with 16 supercharges, because in that case the five- and four-dimensional Weyl multiplets carry the same number of degrees of freedom. See appendix B for details.

To further explore this difference between four and five space-time dimensions, let us also consider the case of small black holes, whose entropy depends sensitively on the higher-derivative couplings. We assume $C_{1 a b}=\eta_{a b}$ and $c_{a}=0$, which represents the typical situation for $\mathrm{K} 3 \times T^{2}$ heterotic string compactifications. From the attractor equations (including the parameter $\alpha$ as before) we obtain

$$
\begin{aligned}
q_{1} & =\frac{6 p^{0}}{\phi^{0^{2}}+p^{0^{2}}}\left[\eta_{a b} \phi^{a} \phi^{b}-\frac{\alpha}{16} c_{1}\right] \\
q_{a} & =\frac{12 p^{0} \phi^{1}}{\phi^{0^{2}}+p^{0^{2}}} \eta_{a b} \phi^{b} .
\end{aligned}
$$

Using the above equations one easily derives,

$$
\begin{aligned}
\mathcal{S}_{\text {macro }}^{\mathrm{BH}} & =\frac{2 \pi p^{0} \phi^{1}}{\left(\phi^{0^{2}}+p^{0^{2}}\right)^{2}}\left[p^{0} q_{1}\left(\phi^{0^{2}}+p^{0^{2}}\right)+\frac{1}{2}\left(\frac{3 \alpha}{4} p^{0^{2}}+\phi^{0^{2}}\right) c_{1}\right], \\
\eta^{a b} q_{a} q_{b} & =\frac{3 p^{0}\left(\phi^{1}\right)^{2}}{\phi^{0^{2}}+p^{0^{2}}}\left[8 q_{1}+\frac{3 \alpha p^{0}}{p^{0^{2}}+\phi^{0^{2}}} c_{1}\right] .
\end{aligned}
$$

Let us now set $q_{1}=0$, so that we are describing small black holes. In that case one finds,

$$
\mathcal{S}_{\text {macro }}^{\mathrm{BH}}=\frac{\pi}{4} \sqrt{\left|\alpha c_{1} \eta^{a b} q_{a} q_{b}\right|}\left\{1+\frac{4-3 \alpha}{3 \alpha} \frac{\phi^{0^{2}}}{\phi^{0^{2}}+p^{0^{2}}}\right\},
$$

where $\phi^{0}$ is related to the angular momentum according to

$$
J_{\psi}=-\frac{\phi^{0}}{\phi^{0^{2}}+p^{0^{2}}} \frac{4-3 \alpha}{12 \alpha} \sqrt{\left|\alpha c_{1} \eta^{a b} q_{a} q_{b}\right|} .
$$

For non-zero angular momentum this last relation does not allow a uniform rescaling of the charges in the way indicated before. For zero angular momentum only the first term in (7.21) contributes. In that case the entropy coincides with the four-dimensional result for small black holes, except for an overall relative factor equal to $\sqrt{4 / 3}$ induced by the $\alpha$-dependence. This result was already discussed in [27] where exact expressions for microscopic degeneracies of small static black holes in five space-time dimensions were derived. 
In this work it was found that the asymptotics of the entropy of the small black holes in five dimensions is the same as in four, with the same normalization. To resolve this puzzle it might perhaps be helpful to also have microscopic results for non-zero angular momentum, so that one has a more detailed test for (7.21). However, such results are quite difficult to obtain. As is well known, in four space-time dimensions the sub-leading contribution to the entropy of small black holes is problematic in the supergravity description, but the leading contribution is in perfect agreement with microstate counting arguments. The five-dimensional result thus poses a puzzle in this respect.

It is also worth mentioning that, when $J_{\psi} \neq 0$, the resulting values of the fourdimensional charges will not correspond to four-dimensional small black holes. Indeed, when setting $\alpha=\frac{4}{3}$ in (7.22), we find $J_{\psi}=0$, so that we are dealing with vanishing $q_{0}, p^{1}, p^{a}$, which do characterize a small black hole in four dimensions. Hence the situation remains unsatisfactory.

\section{BPS black rings}

In this final section we turn to the black rings, for which the relevant Noether potential has been derived in section 6 . In particular we refer to the treatment of the mixed ChernSimons term in subsection 6.1, which is crucial for the black ring. In this section we discuss the resulting expressions for the entropy, and for the charges and angular momenta, which are then confronted with results from the literature. As we shall see, the actual evaluation still involves a number of non-trivial issues related to the integration over the spacelike section $\Sigma$ of the horizon.

The relevant Noether potential consists of (6.8) combined with the contributions from the Chern-Simons terms that can be extracted from (6.11) and (6.16). Using that $T_{01}=0$ for the black ring, it is easy to see that (6.8) gives rise to the following contribution,

$$
8 \pi^{2} \varepsilon_{\mu \nu} \mathcal{Q}_{0}^{\mu \nu}=-2 \varepsilon_{01}\left[C(\sigma)+3 c_{I} \sigma^{I} T_{23}^{2}\right] \nabla_{[0} \xi_{1]} .
$$

Subsequently we add the contributions from (6.16), together with the first term in (6.11) that originates from the $W \wedge F \wedge F$ Chern-Simons term,

$$
\begin{aligned}
8 \pi^{2} \mathcal{Q}_{\mathrm{CS}}^{\mu \nu}= & \frac{1}{2} \mathrm{i} e^{-1} \varepsilon^{\mu \nu \rho \sigma \tau} C_{I J K} \xi^{\lambda} W_{\lambda}^{I} W_{\rho}^{J} F_{\sigma \tau}{ }^{K} \\
& -\frac{1}{32} \mathrm{i} e^{-1} \varepsilon^{\mu \nu \rho \sigma \tau} c_{I} F_{\rho \sigma}{ }^{I} \omega_{\tau}^{a b}\left[\varepsilon_{a b}-\frac{1}{2} \xi^{\kappa} \omega_{\kappa a b}\right] \\
& +\frac{1}{32} \mathrm{i} e^{-1} \varepsilon^{\mu \nu \rho \sigma \tau} c_{I} \xi^{\lambda} W_{\lambda}^{I} \omega_{\rho}^{a b}\left(\partial_{\sigma} \omega_{\tau a b}-\frac{2}{3} \omega_{\sigma a c} \omega_{\tau}^{c} b\right) \\
& -\frac{1}{32} \mathrm{i} e^{-1} \varepsilon^{\rho \sigma \tau \lambda[\mu} c_{I} F_{\rho \sigma}^{I} \mathcal{R}_{\tau \lambda}{ }^{\nu] \kappa} \xi_{\kappa} \\
& +\frac{1}{64} \mathrm{i} e^{-1} \varepsilon^{\rho \sigma \tau \lambda \kappa} c_{I} F_{\rho \sigma}^{I} \mathcal{R}_{\tau \lambda}^{\mu \nu} \xi_{\kappa} .
\end{aligned}
$$

Observe that the last two terms in (8.2) have already been evaluated in (6.11). The third term of (8.2) vanishes as can be readily deduced from (4.42). Straightforwardly combining 
the various contributions gives rise to the following additional contribution to the Noether potential,

$$
\begin{aligned}
8 \pi^{2} \varepsilon_{\mu \nu} \mathcal{Q}_{\mathrm{CS}}^{\mu \nu}= & 2 \varepsilon_{01} T_{23}\left[4 C_{I J K} \sigma^{I} W_{5}{ }^{J} \xi^{\lambda} W_{\lambda}{ }^{K}-c_{I} \sigma^{I} T_{23}{ }^{2} \xi_{5}\right] \\
& -2 \varepsilon_{01} c_{I} \sigma^{I} T_{23}{ }^{2}\left[\nabla_{[0} \xi_{1]}-\frac{1}{2} \xi^{\lambda} \omega_{\lambda 01}\right]
\end{aligned}
$$

where we have used that $\omega_{5}^{a b}$ vanishes with the exception of $\omega_{5}{ }^{01}=-2 T_{23}$.

From (8.3) we directly determine the expression for the entropy, which coincides with the corresponding expression (7.1) for the black hole,

$$
\mathcal{S}_{\text {macro }}^{\text {BR }}=\frac{\pi \mathrm{e}^{g}}{4 v^{2}}\left[C(\sigma)+4 c_{I} \sigma^{I} T_{23}^{2}\right] .
$$

Observe that, in order to obtain this result, it was crucial to use the alternative form of the Noether potential derived in subsection 6.1. Naive application of the Noether potential that was used earlier for the black hole, will yield a different result. In any case, we should stress that the mixed Chern-Simons term contributes to both the black hole and the black string entropy. This raises some question about the derivation presented in [36] where the Chern-Simons term was not taken into account.

To obtain the expression (8.4) we had to integrate over the horizon, which, in the case at hand, was straightforward. However, to determine the electric charges and the angular momenta, one is confronted with an integration of terms that depend explicitly on gauge fields that are not globally defined. To perform the integral one therefore has to make use of patches, as was already explained in section 4.3 , in such a way that the result will be invariant under 'small' gauge transformations continuously connected to the identity. The precise procedure for doing this has already been proposed in [12], and we will adopt it here.

We thus define two coordinate patches on the $S^{1} \times S^{2}$ spacelike cross section $\Sigma$ of the horizon. As we shall discuss in due time, these patches have to be also defined away from $\Sigma$, but for the moment we restrict our attention to $\Sigma$ itself. One patch contains the north pole $\mathrm{N}$ of the $S^{2}$ factor. It is parametrized by $-1+\epsilon \leq \cos \theta \leq 1,0 \leq \varphi<2 \pi$ and $0 \leq \psi<4 \pi$. This patch has the topology of a solid two-torus. The second patch, which has the same topology, contains the south pole $\mathrm{S}$ of the $S^{2}$ factor, and is parametrized by $-1 \leq \cos \theta \leq-1+\epsilon, 0 \leq \varphi<2 \pi$ and $0 \leq \psi<4 \pi$. The boundary of these two patches is a two-torus defined by $\cos \theta=-1+\epsilon$, where the parameter $\epsilon$ will be taken to zero at the end of the calculation. On these patches we define the gauge fields, $W_{\mu}^{\mathrm{N} I}$ and $W_{\mu}^{\mathrm{S} I}$, respectively, which are related by gauge transformations $\beta^{I}$. These gauge transformations move the Dirac brane singularities from the south to the north pole in a way that involves the ring coordinate $\psi$, as was already described in subsection 4.3 (in particular, see (4.36)). Hence,

$$
\begin{aligned}
W_{\mu}^{\mathrm{N} I} \mathrm{~d} x^{\mu} & =-p^{I}\left[\cos \theta \mathrm{d} \varphi-\mathrm{d}\left(\varphi+\frac{1}{2} \psi\right)\right]+a^{I} \mathrm{~d} \psi, \\
W_{\mu}^{\mathrm{S} I} & =W_{\mu}^{\mathrm{N} I}+\beta_{\mu}{ }^{I}, \quad \beta_{\mu}{ }^{I} \mathrm{~d} x^{\mu}=-2 p^{I} \mathrm{~d}\left(\varphi+\frac{1}{2} \psi\right) .
\end{aligned}
$$

Integrals over the spacelike cross section $\Sigma$ of the horizon, are now decomposed into integrals over the sections $\mathrm{N}$ and $\mathrm{S}$ and an additional integral over the boundary of the 
coordinate patches that involves the gauge transformations $\beta^{I}$. This last term must restore the gauge invariance of the integral under small gauge transformations [12]. The limit $\epsilon \downarrow 0$ is taken for convenience, so that the contribution from the section $\mathrm{S}$ will vanish, and the contribution from $\mathrm{N}$ will cover the whole horizon with the exception of the singular points related to the position of the Dirac brane.

Let us first consider the attractor equations for the electric charges $q_{I}$. From the evaluation of the charges for the black hole (c.f. (5.10)) it is clear that the only contribution originates from the $C_{I J K} W^{I} \wedge F^{J} \wedge F^{K}$ Chern-Simons term, since all other contributions vanish when $T_{01}=0$. Therefore we focus directly on the Chern-Simons term, which requires to evaluate the integral of $C_{I J K} W^{J} \wedge F^{K}$ over the spacelike cross section $\Sigma$ of the horizon. According to the prescription specified above, this integral is evaluated as follows,

$$
\begin{aligned}
\int_{\Sigma} C_{I J K} W^{J} \wedge F^{K}= & \int_{\mathrm{N}} C_{I J K} W^{\mathrm{N} J} \wedge F^{K}+\int_{\mathrm{S}} C_{I J K} W^{\mathrm{S} J} \wedge F^{K} \\
& +2 \int_{\partial \mathrm{N}} C_{I J K} W^{\mathrm{N} J} \wedge \beta^{K},
\end{aligned}
$$

where the factor 2 arises because $F^{I}=2 \mathrm{~d} W^{I}$. In the limit $\epsilon \downarrow 0$, the second integral vanishes. The third integral extends over the boundary, $\partial \mathrm{N}=-\partial \mathrm{S}$, of the two sections. Now, observe that $W^{\mathrm{N} J} \wedge F^{K}$ is proportional to $\left(a^{J}+\frac{1}{2} p^{J}\right) p^{K} \mathrm{~d} \theta \wedge \mathrm{d} \varphi \wedge \mathrm{d} \psi$, while $W^{\mathrm{N} J} \wedge \beta^{K}$ is proportional to $\left(a^{J}-\frac{1}{2}(1-\epsilon) p^{J}\right) p^{K} \mathrm{~d} \varphi \wedge \mathrm{d} \psi$. As it turns out, the contributions proportional to $p^{J} p^{K}$ from the first and the second integral cancel (in the limit $\epsilon \downarrow 0$ ), whereas the terms proportional to $a^{J} p^{K}$ add. This confirms the conclusion below (4.37) that the ChernSimons charge should be proportional to $C_{I J K} a^{J} p^{K}$. From comparison with (5.10), one then easily determines the expression for the electric charges by substituting $\left[W_{\psi}{ }^{K}\right]=2 a^{K}$. The attractor equations for the black ring charges are therefore summarized by

$$
q_{I}=-\frac{3}{T_{23}} C_{I J K} \sigma^{J} a^{K}, \quad p^{0}=0, \quad p^{I}=\frac{\sigma^{I}}{4 T_{23}} .
$$

It is important to realize that the prescription of [12] is based on the fact that $\mathrm{d}\left[W^{J} \wedge F^{K}\right]=\frac{1}{2} F^{J} \wedge F^{K}$ is gauge invariant. Upon extending the patches outside the horizon, we may calculate $F^{J} \wedge F^{K}$ over a four-dimensional manifold by extending the radial coordinate $r$, which can then be expressed as an integral over its three-dimensional boundary. This is the justification for the prescription (8.6), as $\Sigma$ constitutes (part of) this boundary. However, we have simply ignored that the gauge fields must in principle be extendable outside the horizon in the two patches, and in the above calculation this feature does not seem to play a role as we obtain a result that is invariant under small gauge transformations. Indeed, one can repeat the calculation without any difficulty for a different choice of coordinate patches, such as, for instance, defined by $\cos \theta_{0} \leq \cos \theta \leq 1$ for the $\mathrm{N}$ patch and $-1 \leq \cos \theta \leq \cos \theta_{0}$ for the $\mathrm{S}$ patch, so that the boundary is located at $\theta=\theta_{0}$. As it turns out the final result will not depend on $\theta_{0}$ and simply remains the same.

However, the situation is different when considering the evaluation of the angular momenta and we shall see that the extension of the sections away from $\Sigma$ will become an issue. The expression for the angular momenta follows from the Noether potential (8.3), 
which is again not gauge invariant so that the integral is again subtle. The troublesome term is the first one, depending on $W_{5}{ }^{J}$, which originates form the $W \wedge F \wedge F$ Chern-Simons term shown in the first line of (8.2). This term leads to

$$
8 \pi^{2} \varepsilon_{\mu \nu} \mathcal{Q}_{\mathrm{CS}}^{\mu \nu}=\varepsilon_{01} \varepsilon^{\mu \nu \rho} C_{I J K} \xi^{\lambda} W_{\lambda}^{I} W_{\mu}{ }^{J} F_{\nu \rho}{ }^{K}+\cdots,
$$

where the dots denote the remaining gauge invariant contributions in (8.3), which can be evaluated straightforwardly. Note that, unlike as on previous occasions, we converted the above expression to a density over $\Sigma$, so that its integration will require only the surface element $\mathrm{d} \psi \wedge \mathrm{d} \varphi \wedge \mathrm{d} \theta$.

In order that the integral over $\Sigma$ of (8.8) is amenable to the same prescription as used above, it is important that $\Sigma$ and the gauge potentials are invariant under the isometries associated with linear combinations, $\xi^{\psi} \partial_{\psi}+\xi^{\varphi} \partial_{\varphi}$, of the two Killing vectors associated with rotations over the angles $\psi$ and $\varphi$. One then observes that $\mathrm{d}[(\xi \cdot W) W \wedge F]$ can be written as a linear combination of two terms. One is the contraction of the Killing vector with the five-form $W \wedge F \wedge F$ whose integral must vanish for symmetry reasons. The second term equals $(\xi \cdot W) F \wedge F$, which changes by a total derivative under gauge transformations, again because the gauge fields are invariant under the symmetry associated with the Killing vector. Hence the integral over the four-dimensional manifold is invariant under small gauge transformations, and, just as before, the integral of (8.8) over its boundary $\Sigma$ can be decomposed into integrals over the patches $\mathrm{N}$ and $\mathrm{S}$ and an additional integral over the boundary $\partial \mathrm{N}$ of

$$
\begin{aligned}
& \varepsilon^{\mu \nu \rho} C_{I J K}\left[\xi^{\lambda} W_{\lambda}^{\mathrm{N} I} W_{\mu}^{\mathrm{N} J}-\xi^{\lambda} W_{\lambda}^{\mathrm{S} I} W_{\mu}^{\mathrm{S} J}\right] F_{\nu \rho}{ }^{K}= \\
& \quad+\varepsilon^{\mu \nu \rho} C_{I J K} \partial_{\mu}\left[\xi^{\lambda} W_{\lambda}^{\mathrm{SI}} \beta_{\nu}{ }^{J} W_{\rho}^{\mathrm{S} K}-2 \xi^{\lambda} \beta_{\lambda}^{I} \beta_{\nu}{ }^{J} W_{\rho}^{\mathrm{S} K}\right] \\
& \quad-\frac{3}{2} \varepsilon^{\mu \nu \rho} C_{I J K} \xi^{\lambda} \beta_{\lambda}^{I} W_{\mu}^{\mathrm{S} J} F_{\nu \rho}{ }^{K} .
\end{aligned}
$$

Here we insisted in writing the last two lines in terms of sections $W_{\mu} \mathrm{S} I$, which are well defined at the south pole. Therefore, when writing the last term as a surface term over $\xi^{\lambda} \beta_{\lambda}^{I} W_{\psi}^{\mathrm{S} J} W_{\varphi}^{\mathrm{S} K}$, its contribution will vanish in the limit $\epsilon \downarrow 0$ because $W_{\varphi}^{\mathrm{S} K}$ vanishes at the south pole.

Combining the results above the integral of (8.8) over $\Sigma$ can therefore be written as,

$$
\begin{aligned}
\int_{\Sigma} \varepsilon_{\mu \nu} \mathcal{Q}_{\mathrm{CS}}^{\mu \nu}= & \frac{\varepsilon_{01}}{4 \pi^{2}} \int \mathrm{d} \theta \mathrm{d} \varphi \mathrm{d} \psi C_{I J K} \xi^{\lambda} W_{\lambda}^{\mathrm{N} I} W_{\psi}^{\mathrm{N} J} F_{\theta \varphi}{ }^{K} \\
& +\left.\frac{\varepsilon_{01}}{4 \pi^{2}} \int \mathrm{d} \varphi \mathrm{d} \psi C_{I J K}\left[\beta_{\varphi}^{I} W_{\psi}^{\mathrm{S} J}-\beta_{\psi}^{I} W_{\varphi}^{\mathrm{S} J}\right]\left(\frac{1}{2} \xi^{\lambda} W_{\lambda}^{\mathrm{S} K}-\xi^{\lambda} \beta_{\lambda}{ }^{K}\right)\right|_{\theta=\pi} .
\end{aligned}
$$

For both of these integrals the limit $\epsilon \downarrow 0$ can be taken without difficulty, so that the first one extends over the whole horizon section $\Sigma$ and the second one over the boundary of the sections on the horizon. A straightforward calculation then leads to $12 C_{I J K} p^{I} p^{J}\left(a^{K}-\frac{1}{6} p^{K}\right)$ and $6 C_{I J K} p^{I}\left(a^{J} a^{K}+a^{J} p^{K}-\frac{1}{12} p^{J} p^{K}\right)$, for $J_{\varphi}$ and $J_{\psi}$, respectively.

The same calculation can be repeated for a different choice of the patches, namely such that, in the limit $\epsilon \downarrow 0$, the S patch will cover the whole horizon area $\Sigma$ and the overlap of 
the $\mathrm{N}$ patch will shrink to the north pole. This requires to re-evaluate (8.9), but up to a few signs the calculations proceeds in the same way. However, now the result is not the same, and one finds instead, $-12 C_{I J K} p^{I} p^{J}\left(a^{K}+\frac{1}{6} p^{K}\right)$ and $6 C_{I J K} p^{I}\left(a^{J} a^{K}-a^{J} p^{K}-\frac{1}{12} p^{J} p^{K}\right)$, for $J_{\varphi}$ and $J_{\psi}$, respectively. The reason for this discrepancy resides in the last term in (8.9), which we dropped because it does not contribute at the south pole of the horizon.

However, one must verify whether there is no obstruction away from the horizon. If one assumes that the south poles are directed to the outward part of the ring, extending all the way to spatial infinity as in [58], one expects an obstruction which will result in an extra contribution from the integral at spatial infinity. On the other hand, for the inner region of the ring which contains the north poles, there is obviously no obstruction, so that the second result will be valid. In case the south poles are directed to the inward part of the ring, it is the first result that would be valid. In other words, a minimal understanding of the topological embedding of the near-horizon region in the global solution is essential in order to distinguish between the two prescriptions. It is possible that only one embedding leads to a solution that is globally BPS, in line with what was found in [39]. For a space that is asymptotically flat, both embeddings seem possible and lead to two inequivalent BPS solutions.

In light of the above we adopt the second result, which must be combined with the contributions from (8.3). Then we obtain the following result for the two independent angular momenta, associated with the two independent rotations of the ring in orthogonal planes,

$$
\begin{aligned}
J_{\varphi} & =-12 C_{I J} p^{I}\left(a^{J}+\frac{1}{6} p^{J}\right) \\
J_{\psi}-J_{\varphi} & =-\frac{\mathrm{e}^{2 g}}{2 T_{23}}\left[C(\sigma)+4 c_{I} \sigma^{I} T_{23}^{2}\right]+6 C_{I J}\left(a^{I}+\frac{1}{2} p^{I}\right)\left(a^{J}+\frac{1}{2} p^{J}\right),
\end{aligned}
$$

where $C^{I J}$ is the inverse of $C_{I J K} p^{K}$.

The above results are all invariant under scale transformations, as they should. Note that the Wilson line moduli $a^{I}$ are scale invariant. As in the case of black holes, we introduce a scale invariant variable,

$$
\phi^{0}=\frac{\mathrm{e}^{-g}}{4 T_{23}}
$$

so that the above expressions for the entropy and the electric charges take a manifestly scale invariant form,

$$
\begin{aligned}
\mathcal{S}_{\text {macro }}^{\mathrm{BR}} & =\frac{4 \pi}{\phi^{0}}\left[C_{I J K} p^{I} p^{J} p^{K}+\frac{1}{4} c_{I} p^{I}\right], \\
q_{I} & =-12 C_{I J K} p^{J} a^{K} .
\end{aligned}
$$

The angular momenta can be expressed as follows,

$$
\begin{aligned}
J_{\psi}-J_{\varphi}-\frac{1}{24} C^{I J}\left(q_{I}-6 C_{I K} p^{K}\right)\left(q_{J}-6 C_{J L} p^{L}\right) & =-\frac{2}{\phi^{0}}\left[C_{I J K} p^{I} p^{J} p^{K}+\frac{1}{4} c_{I} p^{I}\right], \\
J_{\varphi} & =p^{I}\left(q_{I}-\frac{1}{6} C_{I J} p^{J}\right) .
\end{aligned}
$$


The choice of the linear combination of the angular momenta in the first term is motivated by the explicit dimensional reduction of the known two-derivative solution [65], which showed that the rotation of the four-dimensional black hole cannot be identified with a rotation of the $S^{2}$ of the black ring but necessarily involves also a rotation along the ring. Likewise the dimensional reduction is over a circle generated by a simultaneous rotation around the ring and of the $S^{2}$. The corresponding generator equals the linear combination of two angular momenta, $J_{\psi}-J_{\varphi}$, which therefore corresponds to the charge associated with the Kaluza-Klein photon. Hence we introduce a modified charge $\hat{q}_{0}$ in the usual fashion,

$$
\hat{q}_{0}=J_{\psi}-J_{\varphi}-\frac{1}{24} C^{I J}\left(q_{I}-6 C_{I K} p^{K}\right)\left(q_{J}-6 C_{J L} p^{L}\right) .
$$

This expression coincides precisely with the one presented in [35].

With this definition the entropy takes its familiar form $[4,32]$,

$$
\mathcal{S}_{\text {macro }}^{\text {BR }}=2 \pi \sqrt{\left|2 \hat{q}_{0}\left(C_{I J K} p^{I} p^{J} p^{K}+\frac{1}{4} c_{I} p^{I}\right)\right|},
$$

This result for the corrected entropy agrees with the microscopic counting of [28, 29]. However, those results do not yet include the contribution from the higher-derivative couplings. As we shall briefly review below, (8.16) takes the same form as the entropy for a corresponding four-dimensional black hole. Namely, it is proportional to $\sqrt{c_{L} \hat{q}_{0}}$, where $c_{L}$ is the relevant central charge of an underlying $(4,0)$ superconformal field theory that arises when wrapping the M-theory five-brane over a cycle $S^{1} \times P_{4}$, where $P_{4}$ is a holomorphic four-cycle of a Calabi-Yau manifold [32]. The modified momentum along the $S^{1}$ is denoted by $\hat{q}_{0}$. The modification is due to the presence of membrane charges. The subleading contributions are associated with the second Chern class of the Calabi-Yau manifold, and on the field-theory side this induces the higher-derivative couplings [4]. Without these subleading corrections, results for other than Calabi-Yau compactifications have been obtained in [66]. The above results are generally in line with the AdS/CFT results for the black ring attractors $[14,33,34]$.

Let us now confront the above results in more detail with the corresponding results in four space-time dimensions, again based on the function (7.8). Hence we are dealing with a black hole with $p^{0}=0$, which leads to

$$
\mathcal{S}_{4 \mathrm{D}}^{\mathrm{BH}}=-\frac{2 \pi}{\phi^{0}}\left[D_{I J K} p^{I} p^{J} p^{K}+256 d_{I} p^{I}\right]
$$

with

$$
\begin{aligned}
q_{I}{ }^{4 \mathrm{D}} & =\frac{6}{\phi^{0}} D_{I J K} p^{J} \phi^{K}, \\
\hat{q}_{0}{ }^{4 D} \equiv q_{0}{ }^{4 D}+\frac{1}{12} D^{I J} q_{I} q_{J} & =\frac{1}{\phi^{0^{2}}}\left[D_{I J K} p^{I} p^{J} p^{K}+256 d_{I} p^{I}\right],
\end{aligned}
$$

where $D^{I J}$ is the inverse of $D_{I J K} p^{K}$. Just as before this gives rise to

$$
\mathcal{S}_{\text {macro }}^{\mathrm{BH}}=2 \pi \sqrt{\left|\hat{q}_{0}\left(D_{I J K} p^{I} p^{J} p^{K}+256 d_{I} p^{I}\right)\right|} .
$$


As the reader can easily verify, the expressions for $\hat{q}_{0}$ and for the entropy are invariant under the transformations (7.11) with $p^{0}=0$. Also the expression for the charges $q_{I}{ }^{4 \mathrm{D}}$ is consistent with this symmetry as it acts on $\phi^{I}$ according to $\phi^{I} \rightarrow \phi^{I}+k^{I} \phi^{0}$. The latter follows straightforwardly from (7.10).

The same transformations can be considered in the five-dimensional case. In five dimensions there is no electric/magnetic duality but there is spectral flow [67], giving rise to the same transformations, upon replacing $D_{I J K}$ by $-2 C_{I J K}$. These transformations are precisely generated by integer shift of the Wilson line moduli, $a^{I} \rightarrow a^{I}+k^{I}$. Observe that the angular momenta will also transform under these shifts, and we find the following results,

$$
\begin{aligned}
q_{I} & \rightarrow q_{I}-12 C_{I J K} p^{J} k^{K}, \\
J_{\varphi} & \rightarrow J_{\varphi}-12 C_{I J K} p^{I} p^{J} k^{K}, \\
J_{\psi} & \rightarrow J_{\psi}-q_{I} k^{I}-6 C_{I J K} p^{I} p^{J} k^{K}+6 C_{I J K} p^{I} k^{J} k^{K},
\end{aligned}
$$

This shows that $\hat{q}_{0}$ remains invariant.

The difference between (8.18) and (8.15) resides in the shifts of the electric charges proportional to $C_{I J K} p^{J} p^{K}$. The presence of these shifts is consistent with many previous results, both from field theoretic solutions and from microstate counting $[9,12,28,29,35$, $65,67,68]$. The modified charges $q_{I}-6 C_{I J K} p^{J} p^{K}$ in $(8.14)$ are additive. This follows from a calculation similar to the one leading to the attractor equation for $q_{I}$, but now for a configuration of concentric rings. Such a calculation has been performed in [12] and resulted in the equations (4.38) and (4.39) that we discussed earlier. When combined with the attractor equation for $q_{I}$ shown in (8.13), they establish the additivity of the shifted charges. The latter is manifest in the results of $[9,67]$. The modified charges should therefore be used in the microscopic formula of [32] to match with the macroscopic result $(8.14)$, as was already emphasized in $[28,35,67]$. Note, however, that in spite of the qualitative agreement of these conclusions, we should stress that we have adopted a different definition of the electric charges $q_{I}$, which is not based on the asymptotic fall-off of the electric fields at spatial infinity. Therefore the modified charges should be the same, but the electric charges may still be different.

The shifts in the electric charges cannot be removed in the four-dimensional results by a suitable duality transformation of the form (7.11), because that transformation induces shifts that are twice as large. The shifts are related to the terms $\pm \frac{1}{2} p^{I} \mathrm{~d} \psi$ in the gauge field sections in (4.36). From the point of view of subsection 4.3, they arise due to the non-trivial topology of the full five-dimensional space-time. Therefore the four-dimensional black hole should be compared to the reduction of an infinite magnetic string in five dimensions, which is topologically trivial. In that case, both the terms $\pm \frac{1}{2} p^{I} \mathrm{~d} \psi$ in (4.36) and the shifts in the electric charges in (8.11) will be absent, so that one obtains full agreement with the four-dimensional attractor results.

\section{Acknowledgments}

It is a pleasure to acknowledge valuable discussions with Gabriel Cardoso, Jan de Boer, Kevin Goldstein, Kentaro Hanaki, Albrecht Klemm, Per Kraus, Finn Larsen, Thomas 
Mohaupt, George Papadopoulos, Yuji Tachikawa, Paul Townsend and Stefan Vandoren. B.d.W. thanks the École Normale Supérieure in Paris, where part of this work was carried out, for hospitality and the Centre National de la Recherche Scientifique (CNRS) for financial support. The work of S.K. is part of the research program of the 'Stichting voor Fundamenteel Onderzoek der Materie (FOM)', which is financially supported by the 'Nederlandse Organisatie voor Wetenschappelijk Onderzoek (NWO)'. This work has been partly supported by EU contracts MRTN-CT-2004-005104 and MRTN-CT-2004-512194, and by NWO grant 047017015 .

\section{A Conventions}

In the first part of this paper, especially when dealing with spinors, we use Pauli-Källén conventions. Five-dimensional world and tangent-space indices are denoted by $\mu, \nu, \ldots$ and $a, b, \ldots$, respectively, and take the values $1,2, \ldots, 5$. We employ hermitean 4 -by-4 gamma matrices $\gamma_{a}$, which satisfy

$$
\begin{aligned}
C \gamma_{a} C^{-1} & =\gamma_{a}^{\mathrm{T}}, \quad C^{\mathrm{T}}=-C, \quad C^{\dagger}=C^{-1}, \\
\gamma_{a b c d e} & =\mathbf{1} \varepsilon_{a b c d e} .
\end{aligned}
$$

Here $C$ denotes the charge-conjugation matrix and gamma matrices with $k$ multiple indices denote the fully antisymmetrized product of $k$ gamma matrices in the usual fashion, so that we have, for instance, $\gamma_{a} \gamma_{b}=\mathbf{1} \delta_{a b}+\gamma_{a b}$. In view of the last equation of (A.1), gamma matrices with more than two multiple indices are not independent, and can be decomposed into the unit matrix, $\gamma_{a}$ and $\gamma_{a b}$. Note that $C, C \gamma_{a}$ and $C \gamma_{a b}$ constitute a complete basis of 6 antisymmetric and 10 symmetric (unitary) matrices in spinor space. The gamma matrices commute with the automorphism group of the Clifford algebra, $\operatorname{USp}(2 N)$, where $N$ denotes the number of independent spinors. Spinors can be described either as Dirac spinors, or as symplectic Majorana spinors. The latter description has the advantage that it makes the action of the $\operatorname{USp}(2 N)$ R-symmetry group manifest. We will thus employ symplectic Majorana spinors $\psi^{i}$ with $i=1,2, \ldots, 2 N$, subject to the reality constraint,

$$
C^{-1} \bar{\chi}_{i}^{\mathrm{T}}=\Omega_{i j} \chi^{j}
$$

where $\Omega$ is the symplectic $\operatorname{USp}(2 N)$ invariant tensor. The Dirac conjugate is defined by $\bar{\psi}=\psi^{\dagger} \gamma_{5}$. Observe that we adhere to the convention according to which raising or lowering of $\operatorname{USp}(2 N)$ indices is effected by complex conjugation.

The gravitini $\psi_{\mu}^{i}$ and associated supersymmetry parameters $\epsilon^{i}$ transform in the $\mathbf{2} \mathbf{N}$ representation of $\operatorname{USp}(2 N)$. In principle we may also consider spinors transforming under more complicated representations of $\operatorname{USp}(2 N)$. For such a spinor $\chi^{i j \cdots}{ }_{m n \cdots}$ the symplectic Majorana constraint would read

$$
C^{-1}\left(\bar{\chi}_{i j \cdots}{ }^{m n \cdots}\right)^{\mathrm{T}}=\Omega_{i k} \Omega_{j l} \Omega^{m p} \Omega^{n q} \cdots \chi^{k l \cdots}{ }_{p q \cdots},
$$

Of course, the symplectic Majorana condition is defined up to a phase, and we made a specific choice for that in (A.2) and (A.3). For fermionic bilinears, with spinor fields $\psi^{i}$ 
and $\varphi^{i}$ and a spinor matrix $\Gamma$ constructed from products of gamma matrices, we note the following result,

$$
\bar{\psi}_{i} \Gamma \varphi^{j}=-\Omega_{i k} \Omega^{j l} \bar{\varphi}_{l} C^{-1} \Gamma^{\mathrm{T}} C \psi^{k}=\left(\bar{\varphi}_{j} \gamma_{5} \Gamma^{\dagger} \gamma_{5} \psi^{i}\right)^{\dagger}
$$

Hence i $\bar{\psi}_{i} \varphi^{j}, \bar{\psi}_{i} \gamma_{a} \varphi^{j}$ and i $\bar{\psi}_{i} \gamma_{a b} \varphi^{j}$ are pseudo-hermitean (provided $a, b, \ldots=1, \ldots, 4$; in Pauli-Källén convention the time component associated with $a=5$ acquires an extra minus sign) . Generalization of this result to spinors transforming according to more complicated $\mathrm{USp}(2 N)$ representations is straightforward.

Multiplication of symplectic Majorana spinors with spinor matrices $\Gamma$ consisting of products of gamma matrices are not automatically symplectic Majorana spinors. This follows from

$$
{\overline{\Gamma \chi^{i}}}^{\mathrm{T}}=\Omega_{i j} C \gamma_{5}\left(C^{-1} \Gamma^{\mathrm{T}} C\right)^{\dagger} \gamma_{5} \chi^{j}
$$

This means that $\mathrm{i} \gamma_{a} \chi^{i}, \gamma_{a b} \chi^{i}, \mathrm{i} \gamma_{a b c} \chi^{i}, \gamma_{a b c d} \chi^{i}$ are also symplectic Majorana spinors with the same reality phase as (A.2).

\section{B Conformal supergravity in 5 space-time dimensions}

The independent bosonic fields in $N=1$ conformal supergravity multiplet in five spacetime dimensions consist of the fünfbein field $e_{\mu}{ }^{a}$, the $\mathrm{SU}(2)$ gauge fields $V_{\mu}{ }^{i}$, the gauge field $b_{\mu}$ associated with scale transformations, and an anti-symmetric tensor field $T_{a b}$ and a scalar field $D$. Furthermore there are composite gauge fields $\omega_{\mu}{ }^{a b}$ and $f_{\mu}{ }^{a}$ associated with the local Lorentz transformations and the conformal boosts. The independent fermionic fields are the gravitino fields $\psi_{\mu}{ }^{i}$ and an ordinary spinor $\chi^{i}$. The composite gauge field $\phi_{\mu}{ }^{i}$ is associated with the so-called special supersymmetry transformations. Together these fields constitute the Weyl supermultiplet [69-71].

The field content of four-dimensional $N=2$ and five-dimensional $N=1$ supergravity is rather similar, in view of the fact that spinors carry four components in both case, Furthermore the R-symmetry groups are almost the same, and equal to $\mathrm{SU}(2) \times \mathrm{U}(1)$ and $\mathrm{USp}(2)$, respectively. However, the number of degrees of freedom are different, as is shown in table 3. The reason can be understood from the fact that the Weyl multiplet is conjugate to the smallest massive supersymmetry representation containing spin- 2 and spin- $3 / 2$ as the highest spin states. For comparison we also display the situation for the $N=4$ Weyl multiplet in four dimensions, and the $N=2$ Weyl multiplet in five dimensions, with corresponding R-symmetry groups $\mathrm{U}(4)$ and USp(4), respectively. These two multiplets comprise the same number of degrees of freedom.

Open Access. This article is distributed under the terms of the Creative Commons Attribution Noncommercial License which permits any noncommercial use, distribution, and reproduction in any medium, provided the original author(s) and source are credited. 


\begin{tabular}{|lcccc|}
\hline & \multicolumn{2}{c}{8 supercharges } & \multicolumn{2}{c|}{ 16 supercharges } \\
\hline field & $\mathrm{d}=4$ & $\mathrm{~d}=5$ & $\mathrm{~d}=4$ & $\mathrm{~d}=5$ \\
\hline$e_{\mu}{ }^{a}$ & 5 & 9 & 5 & 9 \\
$V_{\mu i}{ }^{j}$ & 9 & 12 & 45 & 40 \\
$A_{\mu}{ }^{[i j]}$ & 3 & - & - & 4 \\
$T_{a b}{ }^{[i j]}$ & 6 & 10 & 36 & 50 \\
$D_{[k l]}{ }^{i j]}$ & 1 & 1 & 20 & 14 \\
$E_{(i j)}$ & - & - & 20 & 10 \\
$\phi$ & - & - & 2 & 1 \\
\hline$\psi_{\mu}{ }^{i}$ & 16 & 24 & 32 & 48 \\
$\chi^{i}[k l]$ & 8 & 8 & 80 & 64 \\
$\Lambda_{i}$ & - & - & 16 & 16 \\
\hline bosons+fermions & $24+24$ & $32+32$ & $128+128$ & $128+128$ \\
\hline
\end{tabular}

Table 3. Bosonic and fermionic degrees of freedom of the Weyl multiplets in four and five dimensions for the case of four and sixteen supercharges. All degrees of freedom can be assigned to a product representation of the group of spatial rotations and the R-symmetry group. Decomposing the states in the second column under the group of 3-dimensional rotations yields a reducible multiplet comprising the states of the four-dimensional Weyl multiplet (given in the first column) and of an extra vector (or tensor) multiplet.

\section{References}

[1] S. Ferrara, R. Kallosh and A. Strominger, N=2 extremal black holes, Phys. Rev. D 52 (1995) 5412 [hep-th/9508072] [SPIRES].

[2] A. Strominger, Macroscopic Entropy of $N=2$ Extremal Black Holes, Phys. Lett. B 383 (1996) 39 [hep-th/9602111] [SPIRES].

[3] S. Ferrara and R. Kallosh, Supersymmetry and Attractors, Phys. Rev. D 54 (1996) 1514 [hep-th/9602136] [SPIRES].

[4] G.L. Cardoso, B. de Wit and T. Mohaupt, Corrections to macroscopic supersymmetric black-hole entropy, Phys. Lett. B 451 (1999) 309 [hep-th/9812082] [SPIRES].

[5] J.C. Breckenridge, R.C. Myers, A.W. Peet and C. Vafa, D-branes and spinning black holes, Phys. Lett. B 391 (1997) 93 [hep-th/9602065] [SPIRES].

[6] J.P. Gauntlett, R.C. Myers and P.K. Townsend, Black holes of D $=5$ supergravity, Class. Quant. Grav. 16 (1999) 1 [hep-th/9810204] [SPIRES].

[7] H. Elvang, R. Emparan, D. Mateos and H.S. Reall, A supersymmetric black ring, Phys. Rev. Lett. 93 (2004) 211302 [hep-th/0407065] [SPIRES].

[8] J.P. Gauntlett, J.B. Gutowski, C.M. Hull, S. Pakis and H.S. Reall, All supersymmetric solutions of minimal supergravity in five dimensions, Class. Quant. Grav. 20 (2003) 4587 [hep-th/0209114] [SPIRES].

[9] J.P. Gauntlett and J.B. Gutowski, General concentric black rings, Phys. Rev. D 71 (2005) 045002 [hep-th/0408122] [SPIRES].

[10] P. Kraus and F. Larsen, Attractors and black rings, Phys. Rev. D 72 (2005) 024010 [hep-th/0503219] [SPIRES]. 
[11] K. Goldstein and R.P. Jena, One entropy function to rule them all, JHEP 11 (2007) 049 [hep-th/0701221] [SPIRES].

[12] K. Hanaki, K. Ohashi and Y. Tachikawa, Comments on charges and near-horizon data of black rings, JHEP 12 (2007) 057 [arXiv:0704.1819] [SPIRES].

[13] K. Hanaki, K. Ohashi and Y. Tachikawa, Supersymmetric Completion of an $R^{2}$ Term in Five- Dimensional Supergravity, Prog. Theor. Phys. 117 (2007) 533 [hep-th/0611329] [SPIRES].

[14] P. Kraus and F. Larsen, Microscopic Black Hole Entropy in Theories with Higher Derivatives, JHEP 09 (2005) 034 [hep-th/0506176] [SPIRES].

[15] A. Castro, J.L. Davis, P. Kraus and F. Larsen, 5D Black Holes and Strings with Higher Derivatives, JHEP 06 (2007) 007 [hep-th/0703087] [SPIRES].

[16] A. Castro, J.L. Davis, P. Kraus and F. Larsen, Precision entropy of spinning black holes, JHEP 09 (2007) 003 [arXiv:0705.1847] [SPIRES].

[17] A. Castro, J.L. Davis, P. Kraus and F. Larsen, String Theory Effects on Five-Dimensional Black Hole Physics, Int. J. Mod. Phys. A 23 (2008) 613 [arXiv:0801.1863] [SPIRES].

[18] A. Sen, Black Hole Entropy Function and the Attractor Mechanism in Higher Derivative Gravity, JHEP 09 (2005) 038 [hep-th/0506177] [SPIRES].

[19] N. Banerjee, I. Mandal and A. Sen, Black Hole Hair Removal, JHEP 07 (2009) 091 [arXiv:0901.0359] [SPIRES].

[20] E. Lozano-Tellechea, P. Meessen and T. Ortín, On $D=4$, 5, 6 vacua with 8 supercharges, Class. Quant. Grav. 19 (2002) 5921 [hep-th/0206200] [SPIRES].

[21] R.M. Wald, Black hole entropy is the Noether charge, Phys. Rev. D 48 (1993) 3427 [gr-qc/9307038] [SPIRES].

[22] T. Jacobson, G. Kang and R.C. Myers, On black hole entropy, Phys. Rev. D 49 (1994) 6587 [gr-qc/9312023] [SPIRES].

[23] V. Iyer and R.M. Wald, Some properties of Noether charge and a proposal for dynamical black hole entropy, Phys. Rev. D 50 (1994) 846 [gr-qc/9403028] [SPIRES].

[24] Y. Tachikawa, Black hole entropy in the presence of Chern-Simons terms, Class. Quant. Grav. 24 (2007) 737 [hep-th/0611141] [SPIRES].

[25] D. Marolf, Chern-Simons terms and the three notions of charge, hep-th/0006117 [SPIRES].

[26] C. Vafa, Black holes and Calabi-Yau threefolds, Adv. Theor. Math. Phys. 2 (1998) 207 [hep-th/9711067] [SPIRES].

[27] M.-x. Huang, A. Klemm, M. Mariño and A. Tavanfar, Black Holes and Large Order Quantum Geometry, Phys. Rev. D 79 (2009) 066001 [arXiv:0704.2440] [SPIRES].

[28] I. Bena and P. Kraus, Microscopic description of black rings in AdS/CFT, JHEP 12 (2004) 070 [hep-th/0408186] [SPIRES].

[29] M. Cyrier, M. Guica, D. Mateos and A. Strominger, Microscopic entropy of the black ring, Phys. Rev. Lett. 94 (2005) 191601 [hep-th/0411187] [SPIRES].

[30] A. Castro and S. Murthy, Corrections to the statistical entropy of five dimensional black holes, JHEP 06 (2009) 024 [arXiv:0807.0237] [SPIRES]. 
[31] N. Banerjee, Subleading Correction to Statistical Entropy for BMPV Black Hole, Phys. Rev. D 79 (2009) 081501 [arXiv:0807.1314] [SPIRES].

[32] J.M. Maldacena, A. Strominger and E. Witten, Black hole entropy in M-theory, JHEP 12 (1997) 002 [hep-th/9711053] [SPIRES].

[33] P. Kraus and F. Larsen, Holographic gravitational anomalies, JHEP 01 (2006) 022 [hep-th/0508218] [SPIRES].

[34] P. Kraus, Lectures on black holes and the $A d S_{3} / C F T_{2}$ correspondence, Lect. Notes Phys. 755 (2008) 193 [hep-th/0609074] [SPIRES].

[35] I. Bena and P. Kraus, $R^{2}$-corrections to black ring entropy, hep-th/0506015 [SPIRES].

[36] M. Guica, L. Huang, W.W. Li and A. Strominger, $R^{2}$ corrections for $5 D$ black holes and rings, JHEP 10 (2006) 036 [hep-th/0505188] [SPIRES].

[37] D. Gaiotto, A. Strominger and X. Yin, New Connections Between 4D and 5D Black Holes, JHEP 02 (2006) 024 [hep-th/0503217] [SPIRES].

[38] K. Behrndt, G. Lopes Cardoso and S. Mahapatra, Exploring the relation between $4 D$ and $5 D$ BPS solutions, Nucl. Phys. B 732 (2006) 200 [hep-th/0506251] [SPIRES].

[39] K. Goldstein and S. Katmadas, Almost BPS black holes, JHEP 05 (2009) 058 [arXiv:0812.4183] [SPIRES].

[40] B. de Wit, J. W. van Holten and A. Van Proeyen, Transformation Rules Of N=2 Supergravity Multiplets, Nucl. Phys. B 167 (1980) 186 [SPIRES].

[41] B. de Wit, J.W. van Holten and A. Van Proeyen, Structure of N=2 supergravity, Nucl. Phys. B 184 (1981) 77 [SPIRES].

[42] B. de Wit and A. Van Proeyen, Potentials and symmetries of general gauged $N=2$ supergravity: Yang-Mills models, Nucl. Phys. B 245 (1984) 89 [SPIRES].

[43] B. de Wit, P.G. Lauwers and A. Van Proeyen, Lagrangians of N=2 Supergravity - Matter Systems, Nucl. Phys. B 255 (1985) 569 [SPIRES].

[44] M. Kaku, P.K. Townsend and P. van Nieuwenhuizen, Properties of Conformal Supergravity, Phys. Rev. D 17 (1978) 3179 [SPIRES].

[45] M. Kaku and P.K. Townsend, Poincaré supergravity as broken conformal supergravity, Phys. Lett. B 76 (1978) 54 [SPIRES].

[46] T. Kugo and S. Uehara, Conformal and Poincaré Tensor calculi in $N=1$ supergravity, Nucl. Phys. B 226 (1983) 49 [SPIRES].

[47] E. Bergshoeff, E. Sezgin and A. Van Proeyen, Superconformal tensor calculus and matter couplings in six-dimensions, Nucl. Phys. B 264 (1986) 653 [SPIRES].

[48] E. Bergshoeff et al., Weyl multiplets of $N=2$ conformal supergravity in five dimensions, JHEP 06 (2001) 051 [hep-th/0104113] [SPIRES].

[49] T. Fujita and K. Ohashi, Superconformal tensor calculus in five dimensions, Prog. Theor. Phys. 106 (2001) 221 [hep-th/0104130] [SPIRES].

[50] E. Bergshoeff et al., $N=2$ supergravity in five dimensions revisited, Class. Quant. Grav. 21 (2004) 3015 [hep-th/0403045] [SPIRES].

[51] B. de Wit, B. Kleijn and S. Vandoren, Superconformal hypermultiplets, Nucl. Phys. B 568 (2000) 475 [hep-th/9909228] [SPIRES]. 
[52] B. de Wit, M. Roček and S. Vandoren, Hypermultiplets, hyperKähler cones and quaternion-Kähler geometry, JHEP 02 (2001) 039 [hep-th/0101161] [SPIRES].

[53] A.F. Swann, Hyperkähler and quaternionic Kähler geometry, Math. Ann. 289 (1991) 421.

[54] G. Lopes Cardoso, B. de Wit, J. Käppeli and T. Mohaupt, Stationary BPS solutions in $N=2$ supergravity with $R^{2}$ interactions, JHEP 12 (2000) 019 [hep-th/0009234] [SPIRES].

[55] P. Krtous, D. Kubiznak, D.N. Page and V.P. Frolov, Killing-Yano tensors, rank-2 Killing tensors and conserved quantities in higher dimensions, JHEP 02 (2007) 004 [hep-th/0612029] [SPIRES].

[56] M. Henneaux and C. Teitelboim, Quantization of topological mass in the presence of a magnetic pole, Phys. Rev. Lett. 56 (1986) 689 [SPIRES].

[57] X. Bekaert and A. Gomberoff, Quantization of the Chern-Simons coupling constant, JHEP 01 (2003) 054 [hep-th/0212099] [SPIRES].

[58] R. Emparan and H.S. Reall, Black rings, Class. Quant. Grav. 23 (2006) R169 [hep-th/0608012] [SPIRES].

[59] X. Bekaert, Issues in electric-magnetic duality, hep-th/0209169 [SPIRES].

[60] N.V. Suryanarayana and M.C. Wapler, Charges from Attractors, Class. Quant. Grav. 24 (2007) 5047 [arXiv: 0704.0955] [SPIRES].

[61] K. Copsey and G.T. Horowitz, The role of dipole charges in black hole thermodynamics, Phys. Rev. D 73 (2006) 024015 [hep-th/0505278] [SPIRES].

[62] G. Lopes Cardoso, B. de Wit and T. Mohaupt, Macroscopic entropy formulae and non-holomorphic corrections for supersymmetric black holes, Nucl. Phys. B 567 (2000) 87 [hep-th/9906094] [SPIRES].

[63] B. de Wit, V. Kaplunovsky, J. Louis and D. Lüst, Perturbative couplings of vector multiplets in N=2 heterotic string vacua, Nucl. Phys. B 451 (1995) 53 [hep-th/9504006] [SPIRES].

[64] B. de Wit and F. Saueressig, Off-shell $N=2$ tensor supermultiplets, JHEP 09 (2006) 062 [hep-th/0606148] [SPIRES].

[65] H. Elvang, R. Emparan, D. Mateos and H.S. Reall, Supersymmetric 4 D rotating black holes from 5D black rings, JHEP 08 (2005) 042 [hep-th/0504125] [SPIRES].

[66] M. Bertolini and M. Trigiante, Microscopic entropy of the most general four-dimensional BPS black hole, JHEP 10 (2000) 002 [hep-th/0008201] [SPIRES].

[67] I. Bena, P. Kraus and N.P. Warner, Black rings in Taub-NUT, Phys. Rev. D 72 (2005) 084019 [hep-th/0504142] [SPIRES].

[68] I. Bena and N.P. Warner, One ring to rule them all ... and in the darkness bind them?, Adv. Theor. Math. Phys. 9 (2005) 667 [hep-th/0408106] [SPIRES].

[69] B. de Wit and J.W. van Holten, Multiplets of linearized SO(2) supergravity, Nucl. Phys. B 155 (1979) 530.

[70] E. Bergshoeff, M. de Roo and B. de Wit, Extended Conformal Supergravity, Nucl. Phys. B 182 (1981) 173 [SPIRES].

[71] P.S. Howe and U. Lindstrom, The supercurrent in five-dimensions, Phys. Lett. B 103 (1981) 422 [SPIRES]. 